\title{
CHARACTERIZATION OF MOLECULARLY \\ IMPRINTED POLYMER PARTICLES SPECIFICALLY \\ TAILORED FOR REMOVAL OF TRACE \\ 17ק-ESTRADIOL IN WATER
}

By

\section{Zackery DeMaleki}

A Thesis submitted to the Faculty of Graduate Studies and Research in Partial Fulfillment of the Requirement for the Degree of Master of Science

\author{
Department of Chemistry \\ Carleton University \\ Ottawa, Ontario
}

Dec. 2009

(C) Copyright

Zackery DeMaleki 


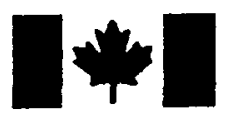

\author{
Library and Archives \\ Canada \\ Published Heritage \\ Branch \\ 395 Wellington Street \\ Ottawa ON K1A ON4 \\ Canada
}

Bibliotheque et

Archives Canada

Direction du

Patrimoine de l'édition

395 , rue Wellington

Ottawa ON K1A ON4

Canada
Your file Votre reférence

ISBN: 978-0-494-64435-5

Our file Notre référence

ISBN: 978-0-494-64435-5
NOTICE:

The author has granted a nonexclusive license allowing Library and Archives Canada to reproduce, publish, archive, preserve, conserve, communicate to the public by telecommunication or on the Internet, loan, distribute and sell theses worldwide, for commercial or noncommercial purposes, in microform, paper, electronic and/or any other formats.

The author retains copyright ownership and moral rights in this thesis. Neither the thesis nor substantial extracts from it may be printed or otherwise reproduced without the author's permission.
AVIS:

L'auteur a accordé une licence non exclusive permettant à la Bibliothèque et Archives Canada de reproduire, publier, archiver, sauvegarder, conserver, transmettre au public par télécommunication ou par l'Internet, prêter, distribuer et vendre des thèses partout dans le monde, à des fins commerciales ou autres, sur support microforme, papier, électronique et/ou autres formats.

L'auteur conserve la propriété du droit d'auteur et des droits moraux qui protège cette thèse. Ni la thèse ni des extraits substantiels de celle-ci ne doivent être imprimés ou autrement reproduits sans son autorisation.
In compliance with the Canadian Privacy Act some supporting forms may have been removed from this thesis.

While these forms may be included in the document page count, their removal does not represent any loss of content from the thesis.
Conformément à la loi canadienne sur la protection de la vie privée, quelques formulaires secondaires ont été enlevés de cette thèse.

Bien que ces formulaires aient inclus dans la pagination, il n'y aura aucun contenu manquant.

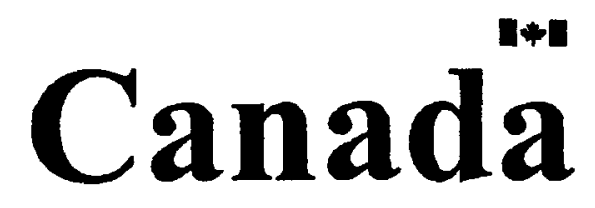




\section{ACKNOWLEDGMENTS}

This thesis arose in part out of years of research that has been done, since I came to Dr. Lai's research group. By that time, I have worked with the number of people whose contribution in assorted ways to the research and the making of the thesis deserved special mention. It is a pleasure to express my gratitude to them all in my humble acknowledgment.

In the first place, I would like to record my gratitude to Dr. Edward P.C. Lai for his extraordinary experiences throughout the work. Above all and the most needed, he provided me unflinching encouragement and support in various ways. His truly scientist intuition has made him as a constant oasis of ideas and passions in science, which exceptionally inspire and enrich my growth as a student, a researcher and a scientist want to be. I am indebted to him more than he knows.

I gratefully acknowledge Dr. Ewa Dabek-Zlotorzynska from Environment Canada for the $\mathrm{CE}$ instrument and providing us chemical whenever needed. Her valuable advice made her a backbone of this research and so on this thesis.

Exceptional thanks also to my entire present and previous group mate specially; Mark Pereira, Amy Hrdina, Patric Young, Min Liu, Shuyi Wu, Woomee Cho, Anastasia Dzhoun, and Farah Hasson, for the valuable advice and great effort on collecting data.

Many thanks go in particular to Audrey Murray who provided me awesome information in using Microsoft Office ${ }^{\circledR}$, which made life much easier.

I gratefully thank Dr. Robert Burk, Maria DeRosa, Banu Örmeci and Maxim Berezovski for their constructive comments on this thesis. I am thankful that the midst of all their activity, 
they accepted to be the member of the committee. I am very grateful for Dr. Sundararajan who opened the new way to success by offering me the Q-year program in the first place. I would also acknowledge to Marilyne Stock and Chantelle Gravelle for all the trouble I had for them during these years.

Where would I be without my family? My Mother is the one who sincerely raised me with her caring and gently love deserves special mention for her inseparable support and prayers. My Father who is always alive in my heart, and my Sister thank you all.

Words fail me to express my appreciation to my wife Negar whose dedication, love and let her intelligence, passions, and ambitions collide with mine. Therefore, I would also thank my family in-law for letting me take her hand in marriage, and accepting me as a member of family and their support. Finally, thank to my Son, Ryan, who I missed too many times joy of being with him and playing with him, to do my study work. 


\section{TABLE OF CONTENTS}

TITLE

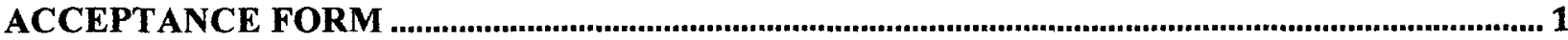

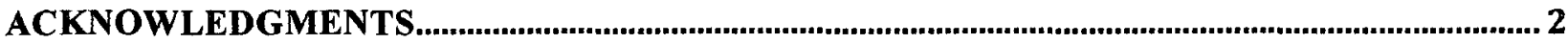

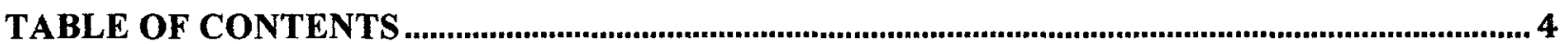

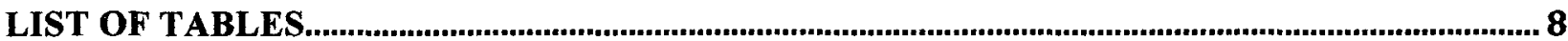

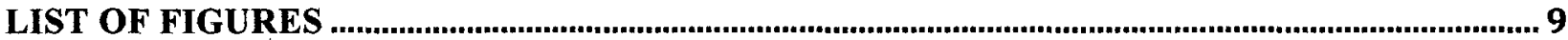

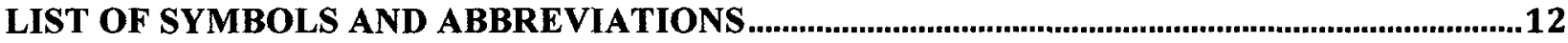

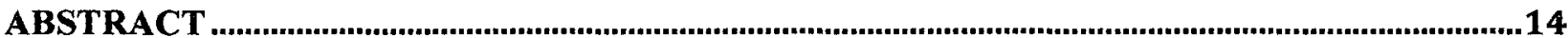

\section{CHAPTER I}

INTRODUCTION

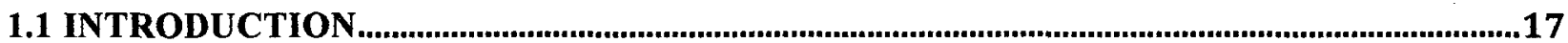

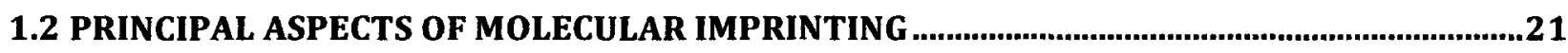

1.2.1 TEMPLATE

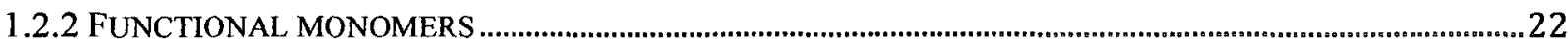

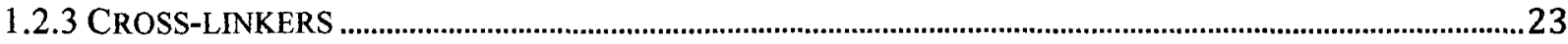

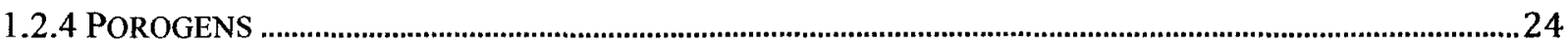

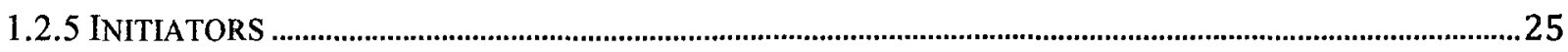

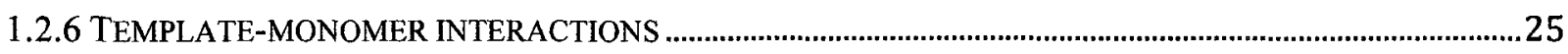

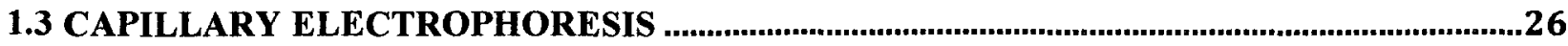

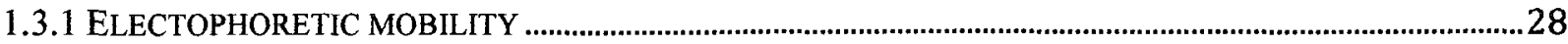




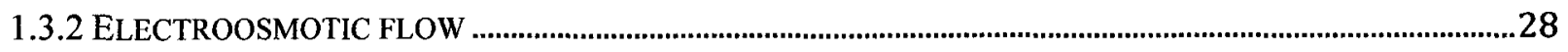

1.3.3 APPARENT MOBILITY

\section{CHAPTER II \\ EXPERIMAENTAL}

2.1 SYNTHESIS AND PROPERTIES OF MIP AND NIP PARTICLES .................................................32

2.1.1 PREPARATION OF MIP AND NIP SUBMICRON PARTICLES ....................................................................32

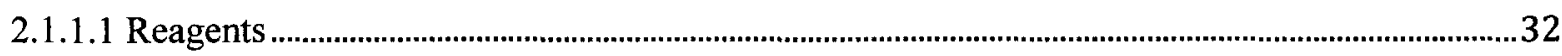

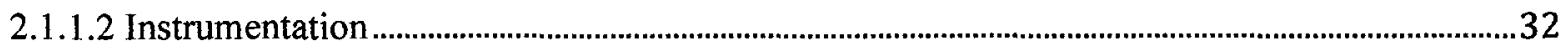

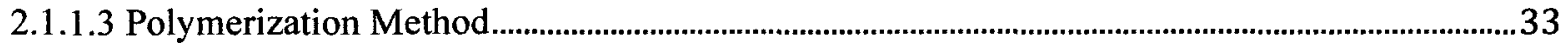

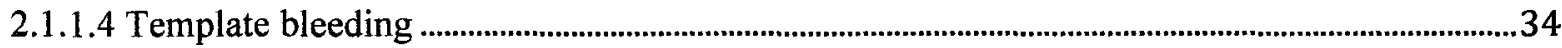

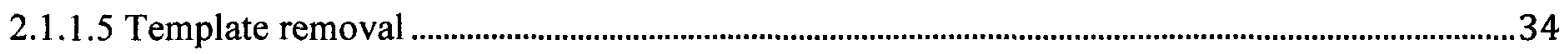

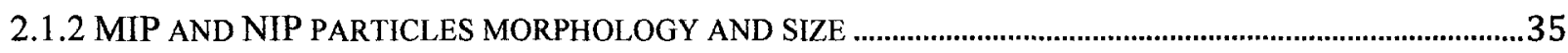

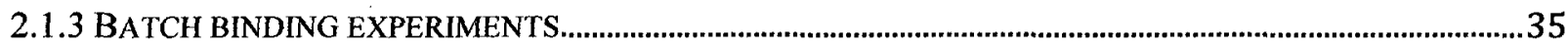

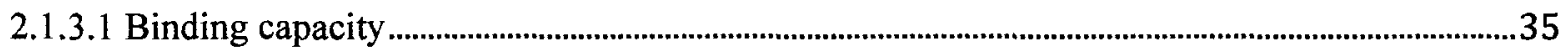

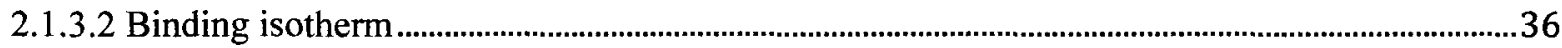

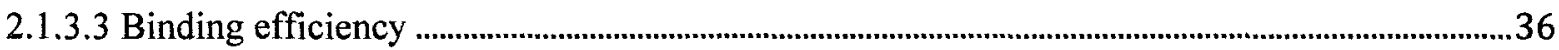

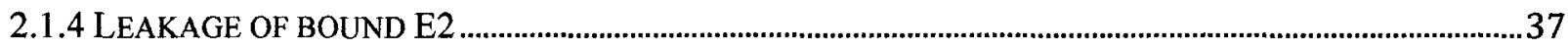

2.2 CAPILLARY ELECTROPHORESIS FOR CHARACTERIZATION OF MIP AND NIP PARTICLES.38

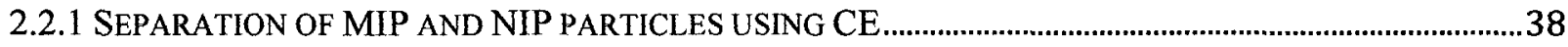

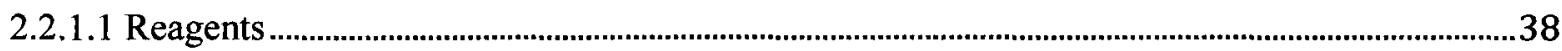

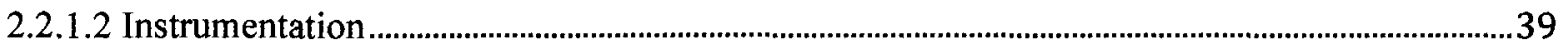

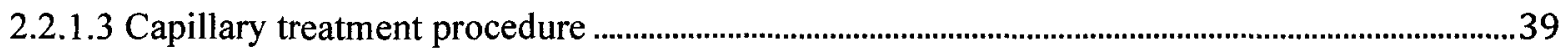

2.2.2 ADDITION OF CAPILLARY MODIFIER AND SEPARATION OF MIP AND NIP PARTICLES ........................40

2.2.3 KINETIC STUDY OF MIP AND NIP PARTICLES BINDING WITH BISPHENOL A .........................................40

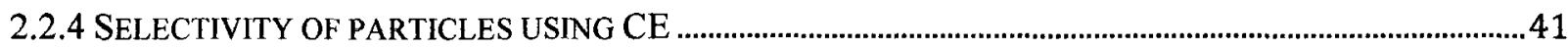


2.2.4.1 Selectivity of MIP-T4 particles on E2 in presence of BPA ...........................................................41

2.2.4.2 Selectivity of MIP-T7 particles on E2 in presence of BPA .........................................................41

2.2.4.3 Non-specific binding of BPA on MIP-T4 and MIP-T7 after binding with E2 $\ldots . . . . . . . . . . . . . . . . . . . . .41$

2.2.4.4 Binding competition of E2 and BPA on MIP-T4 and MIP-T7 particles ....................................42

\section{CHAPTER III}

RESULTS AND DISCUSSIONS

3.1 SYNTHESIS AND PROPERTIES OF MIP AND NIP PARTICLES …..........................................45

3.1.1 PREPARATION OF MIP AND NIP SUBMICRON PARTICLES ..................................................................45

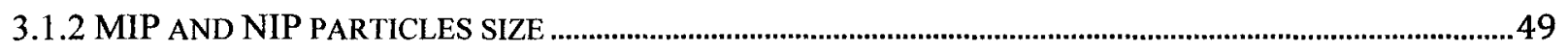

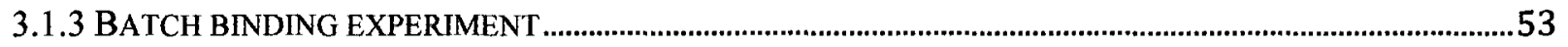

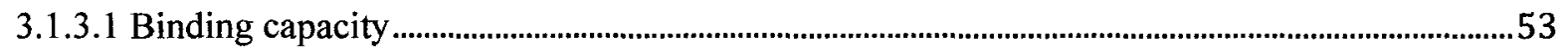

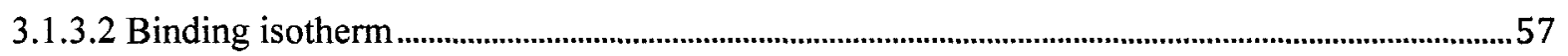

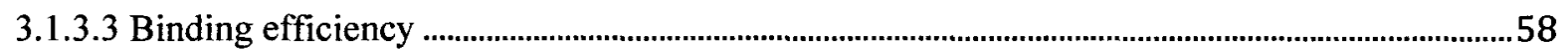

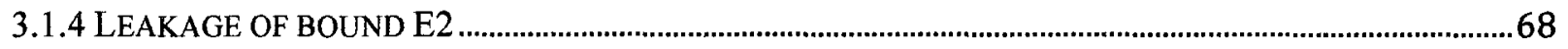

\subsection{CAPILLARY ELECTROPHORESIS FOR CHARACTERIZATION OF MIP AND NIP PARTICLES.71}

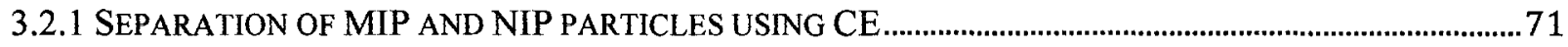

3.2.2 ADDITION OF DYNAMIC CAPILLARY MODIFIER AND SEPARATION OF MIP AND NIP PARTICLES ...75

3.2.2.1 Characterization of MIP and NIP particles based on the size and charge ..................................80

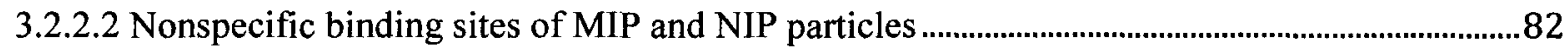

3.2.3 KINETIC STUDY OF MIP AND NIP PARTICLES BINDING WITH BISPHENOL A ......................................83

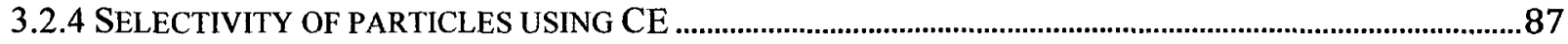

3.2.4.1 Selectivity of MIP-T4 particles on E2 in presence of BPA .........................................................87

3.2.4.2 Selectivity of MIP-T7 particles for E2 in presence of BPA ….......................................................

3.2.4.3 Non-specific binding of BPA onto MIP-T4 and MIP-T7 after binding with E2 .......................95

3.2.4.4 Binding competition of E2 and BPA on MIP-T4 and MIP-T7 particles....................................98 


\section{CHAPTER IV}

\section{CONCLUSIONS}

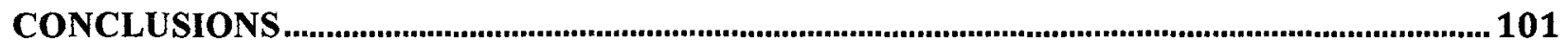

FUTURE WORK

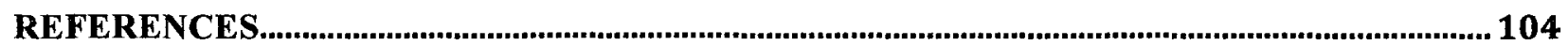




\section{LIST OF TABLES}

Table 1 Composition of pre-polymerization mixture for the synthesis of molecularly imprinted polymers, specifically for E2.

Table 2 Sample preparation for characterization of binding mechanisms of MIP particles. 42

Table 3 Sample preparation for binding competition of E2 and BPA on MIP particles. 43

Table 4 Diameters of different synthesized polymer obtained with DLS and SEM. 52

Table 5 Association constant and binding capacities of high-affinity and low-a affinity binding sites of particles by scatchard data analysis.

Table 6 Amount of bound E2 per gram of MIP-E particles. 64

Table 7 Amount of bound E2 per gram of MIP-T4 and MIP-T7 particles. 65

Table 8 amount of bound and leaked E2 on MIP-T7 and MIP-T4 particles. 70

Table 9 Electrophoretic mobilities of MIP and NIP particles before and after addition of dynamic coating modifier.

Table 10 Resolution of separation of MIP and NIP particles before and after addition of dynamic coating modifier.

Table 11 Amounts of bound BPA on MIP-T4 and NIP-T4 particles in presence of E2. 89

Table 12 Amount of bound BPA on MIP-T7 and NIP-T7 particles in presence of E2. 93

Table 13 Amount of bound BPA on MIP-T7 and NIP-T7 in presence of different concentrations of BPA. 


\section{LIST OF FIGURES}

Figure 1 Structures of a) acrylic acid, b) methacrylic acid.

Figure 2 Structures of a) 17ß-estradiol, b) bisphenol A, c) ethyleneglycol dimethacrylate and d) trimethylolpropane trimethacrylate.

Figure 3 Polimerization of MAA after decomposition of AIBN to produce a free radical. 25

$\begin{array}{ll}\text { Figure } 4 \text { Schematic preparation of MIP particles. } & 26\end{array}$

Figure 5 Typical instrumental set up of capillary electrophoresis. 27

Figure 6 The double-layer charge distribution at a negatively charged capillary wall leading to a generation of EOF. $\quad 29$

Figure 7 Bleeding template in MIP-T7 $(\diamond)$ and MIP-T4 $(\diamond)$ particles before removal of template. 48

Figure 8 SEM image of a) MIP-E, b) NIP-E, magnification of $35 \mathrm{kX}$, acceleration voltage $20 \mathrm{kV}$. 50

Figure 9 Typical effective size distribution of MIP-E particles obtained by nano- DLS particle size analyzer.

Figure 10 SEM images of (a) MIP-T7, (b) NIP-T7, (c) MIP-T4, and (d) NIP-T4. Magnification of 35 kX was afforded by acceleration voltage of $20 \mathrm{kV}$.

Figure 11 Scatchard plots for determination of binding properties of MIP-E $(\bullet)$ and NIP-E $(\bullet)$. 54

Figure 12 Scatchard plots for determination of binding properties of MIP-T7 $(\bullet)$ and NIP-T7 $(\diamond)$. 55

Figure 13 Scatchard plots for determination of binding properties of MIP-T4 $(\bullet)$ and NIP-T4 $(\diamond) . \quad 56$

Figure 14 Determination of optimum amount of MIP-E for binding E2. 58

Figure 15 Binding efficiency of MIP-E after $2(\bullet), 15(\diamond)$ and $60(\diamond)$ minutes incubation. 59

Figure 16 Binding efficiency of $20 \mathrm{mg}$ MIP-E $(\diamond)$ and NIP-E $(\diamond)$ after 2 minutes incubation. $\quad 60$

Figure 17 Binding efficiency of 5mg MIP-T7 $(\bullet)$ and NIP-T7 $(\bullet)$ after 2 minutes incubation. 61

Figure 18 Binding efficiency of 5mg MIP-T4 $(\bullet)$ and NIP-T4 $(\bullet)$ after 2 minutes incubation. 62

Figure 19 Binding efficiency of $0.5 \mathrm{mg}$ MIP-E after 2 minutes incubation. 63

Figure 20 Binding efficiency of $0.5 \mathrm{mg}$ MIP-T7 after 2 minutes incubation. 65 
Figure 21 Binding efficiency of $0.5 \mathrm{mg}$ MIP-T4 after 2 minutes incubation.

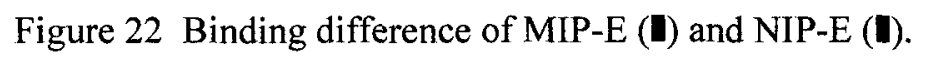

Figure 23 Binding difference of MIP-T7 (U) and NIP-T7 (I).

Figure 24 Binding difference of MIP-T4 (I) and NIP-T4 (I).

Figure 25 HPLC chromatograms of supernatant after 6 hours incubation of MIP-T4 particles in pure water.

Figure 26 HPLC chromatograms $0.05 \mu \mathrm{g} / \mathrm{mL}$ of E2 in Aqueous solution.

Figure 27 CE electropherograms of (a) MIP-E, (b) NIP-E, (c) MIP-T4, (d) NIP-T4, (e) MIP-T7, (f) NIP$\mathrm{T} 7$ particles, in $50 \mathrm{mM}$ borate buffer $(\mathrm{pH}=8.5)$, separation voltage $30 \mathrm{kV}$; detection, $\mathrm{UV}$ at $214 \mathrm{~nm}$.

Figure 28 Separation of MIP-T4 and NIP-T4 by CE in $50 \mathrm{mM}$ borate buffer $(\mathrm{pH}=8.5)$.

Figure 29 Separation of MIP-T7 and NIP-T7 by CE in $50 \mathrm{mM}$ borate buffer ( $\mathrm{pH}=8.5)$.

Figure 30 Separation of MIP-E and NIP-E by CE in $50 \mathrm{mM}$ borate buffer $(\mathrm{pH}=8.5)$.

Figure 31 Electroosmotic mobility measured for several EOTrol ${ }^{\mathrm{TM}}$ dynamic coatings as a function of buffer $\mathrm{pH}$ in CZE.

Figure 32 UV-VIS spectra of different EOTrol ${ }^{\mathrm{TM}}$ dynamic coating modifiers in water.

Figure $33 \mathrm{CE}$ electrophoresis of a) MIP-T4 and b) NIP-T4, in $50 \mathrm{mM}$ borate buffer $(\mathrm{pH}=8.5)$ containing $0.005 \% \mathrm{w} / \mathrm{v}$ EOTrol $^{\mathrm{TM}} \mathrm{LN}$, separation voltage $-30 \mathrm{kV}$; detection, $U \mathrm{~V}$ at $214 \mathrm{~nm}$.

Figure $34 \mathrm{CE}$ electrophoresis of a) MIP-T7 and b) NIP-T7, in $50 \mathrm{mM}$ borate buffer ( $\mathrm{pH}=8.5$ ) containing $0.005 \% \mathrm{w} / \mathrm{v}$ EOTrol ${ }^{\mathrm{TM}} \mathrm{LN}$, separation voltage $-30 \mathrm{kV}$; detection, UV at $214 \mathrm{~nm}$.

Figure $35 \mathrm{CE}$ electrophoresis of 1:1 mixture of $1 \mathrm{mg} / \mathrm{mL}$ MIP-T4 and NIP-T4 particles, in $50 \mathrm{mM}$ borate buffer ( $\mathrm{pH}=8.5$ ) containing $0.005 \% \mathrm{w} / \mathrm{v}$ EOTrol $^{\mathrm{TM}} \mathrm{LN}$, under separation voltage of $-30 \mathrm{kV}$, using UV detection at $214 \mathrm{~nm}$.

Figure $36 \mathrm{CE}$ electrophoresis of 1:1 mixture of $1 \mathrm{mg} / \mathrm{mL}$ MIP-T7 and NIP-T7, in $50 \mathrm{mM}$ borate buffer $(\mathrm{pH}=8.5)$ containing $0.005 \% \mathrm{w} / \mathrm{v}$ EOTrol ${ }^{\mathrm{TM}} \mathrm{LN}$, separation voltage $-30 \mathrm{kV}$; detection, UV at $24 \mathrm{~nm}$. 
Figure $37 \mathrm{CE}$ of $1 \mathrm{mg} / \mathrm{mL}$ MIP-E and $1 \mathrm{mg} / \mathrm{mL}$ NIP-E, in $50 \mathrm{mM}$ borate buffer $(\mathrm{pH}=8.5)$ containing $0.005 \% \mathrm{w} / \mathrm{v}$ EOTrol ${ }^{\mathrm{TM}} \mathrm{LN}$; separation voltage: $-30 \mathrm{kV}$; UV detection at $24 \mathrm{~nm}$.

Figure 38 CE electropherograms of (a) $20 \mu \mathrm{g} / \mathrm{mL}$ BPA, and (b) $1 \mathrm{mg} / \mathrm{mL}$ of NIP-T4 in $20 \mu \mathrm{g} / \mathrm{mL}$ BPA after $29 \mathrm{~s}$ of incubation.

Figure $39 \%$ binding of BPA after adding $1 \mathrm{mg} / \mathrm{mL}$ NIP-T4 into $20 \mu \mathrm{g} / \mathrm{mL}$ of BPA for $20-120 \mathrm{~s} . \quad 85$

Figure $40 \%$ binding of BPA after adding $1 \mathrm{mg} / \mathrm{mL}$ NIP-T7 into $20 \mu \mathrm{g} / \mathrm{mL}$ of BPA for $20-120 \mathrm{~s}$. 86

Figure 41 CE electropherograms of NIP-T4 before $(-)$ and after $(-)$ binding with BPA.

Figure 42 Binding efficiency of BPA on MIP-T4 particles in presence of different concentrations of E2. Concentration of BPA was $20 \mu \mathrm{g} / \mathrm{mL}$.

Figure 43 Binding efficiency of BPA on NIP-T4 particles in presence of different concentrations of E2. Concentration of BPA was $20 \mu \mathrm{g} / \mathrm{mL}$.

Figure 44 Binding efficiency of BPA MIP-T4 $(\bullet)$ and NIP-T4 $(\diamond)$ in presence of different concentrations of BPA.

Figure 45 Binding efficiency of BPA on MIP-T7 particles in presence of different concentrations of E2.

Figure 46 Binding efficiency of BPA on NIP-T7 particles in presence of different concentrations of E2.94

Figure 47 Binding efficiency of BPA on MIP-T7 $(\bullet)$ and NIP-T7 $(\bullet)$ in presence of different concentrations of BPA.

Figure 48 Binding efficiency of BPA on MIP-T4 particles, 20 seconds after addition of E2 at various concentrations. Concentration of BPA was $20 \mu \mathrm{g} / \mathrm{mL}$.

Figure 49 Binding efficiency of BPA on MIP-T7 particles, 20 seconds after addition of E2 at various concentrations. Concentration of BPA was $20 \mu \mathrm{g} / \mathrm{mL}$.

Figure 50 Binding efficiency of BPA $(20 \mu \mathrm{g} / \mathrm{mL})$ on MIP-T4 particles, determined after addition of E2 at various concentrations to compete for binding sites.

Figure 51 Binding efficiency of BPA $(20 \mu \mathrm{g} / \mathrm{mL})$ on MIP-T7 particles, determined after addition of E2 at various concentrations to compete for binding sites. 


\section{LIST OF SYMBOLS AND ABBREVIATIONS}

\begin{tabular}{ll} 
AIBN & 2,2 '-azobisisobutyronitrile \\
BGE & Background electrolyte \\
BPA & Bisphenol A \\
CE & Capillary electrophoresis \\
DDW & Distilled deionized water \\
DLS & Dynamic light scattering \\
E1 & Estrone \\
E2 & $17 \beta$-Estradiol \\
E3 & Estriol \\
EDC & Endocrine disturbing compounds \\
EE2 & $17 \alpha$-Ethynylestradiol \\
EGDMA & Ethyleneglycol dimethacrylate \\
EOF & Electroosmotic flow \\
FD & Florescence detector \\
FFF & Field flow fractionation \\
GE & Gel electrophoresis \\
HPLC & High pressure liquid chromatography \\
LDL & Low-density lipoprotein \\
LN & Low normal \\
MAA & Methacrylic acid \\
\hline
\end{tabular}




$\begin{array}{ll}\text { MIP } & \text { Molecularly imprinted polymer } \\ \text { NIP } & \text { Non-imprinted polymer } \\ \text { SEC } & \text { Size exclusion chromatography } \\ \text { SEM } & \text { Scanning electron microscopy } \\ \text { SPE } & \text { Solid phase extraction } \\ \text { TEA } & \text { Triethylamine } \\ \text { TRIM } & \text { Trimethylol propane trimethacrylate } \\ \text { UV } & \text { Ultra-violet } \\ \text { WWTP } & \text { Wastewater treatment plants }\end{array}$




\begin{abstract}
Endocrine disturbing chemicals (EDCs) are a group of emerging contaminants that have been detected at trace concentrations in water around the world. Natural and synthetic hormones, (including estrogenic compounds) from the EDCs family in water and wastewater effluents have an adverse impact on ecology and human health. This poses a unique challenge for wastewater treatment plants (WWTP) intent on the analysis and removal of estrogenic compounds. During the last decade, research interest in molecularly imprinted polymer (MIP) particles has increased strongly. Chemical and mechanical stability together with high selectivity for the template analyte render MIPs an interesting alternative to routinely applied sorbent materials or antibodies.
\end{abstract}

This thesis consists of two main studies on the preparation of MIP particles specifically for $17 \beta$-estradiol (E2), and the characterization of MIP particles using capillary electrophoresis (CE). Three types of MIP particles were synthesized, using either EGDMA (MIP-E) or TRIM (MIP-T4, MIP-T7) in two different mole ratios as a cross-linker. Binding efficiency of E2 on these MIP and non-imprinted polymer (NIP) particles were examined. Experimental results showed that after 2 min of incubation, $90 \pm 5 \%$ of E2 bound onto the particles. According to Scatchard analysis, an adsorption model containing two types of binding sites, namely specific and non-specific, was proposed. Further results indicated that the binding capacity of $8 \pm 3$ $\mu \mathrm{mol} / \mathrm{g}$ for MIP-E was increased significantly to $33 \pm 7$ and $43 \pm 8 \mu \mathrm{mol} / \mathrm{g}$ for MIP-T4 and MIP-T7. Leakage tests, by high performance liquid chromatography with fluorescence detection (HPLCFD), showed that bound E2 did not leak to the aqueous solution. The binding capacity, high binding efficiency of $96 \pm 3 \%$ (after equilibrium) and no detectable leakage of bound E2 made these particles a great kind of sorbent materials for removal of E2 from water. 
CE characterization of MIP and NIP particles was investigated next. Electropherograms were successfully acquired for the six different MIP and NIP particles, and their electrophoretic mobilities were determined. Separation of MIP and NIP particles using $50 \mathrm{mM}$ borate buffer (pH 8.5) containing $0.005 \%(\mathrm{w} / \mathrm{v})$ EOTrol ${ }^{\mathrm{TM}} \mathrm{LN}$ allowed CE for use in reverse polarity $(-30 \mathrm{kV})$ as a simple and fast characterization technique. Dynamic light scattering (DLS) measurement of the sizes, together with the electrophoretic mobilities of MIP and NIP particles, allowed for an estimation of their surface charges. These charges were interpreted to represent the nonspecific binding properties of MIP in comparison with NIP particles. Instrumental limitation for injection of E2 (or other EDCs) and NIP particles in mixture set a lower limit (of $\sim 20 \mathrm{~s}$ ) on allowable incubation time for non-specific binding kinetics study. Co-injection of bisphenol A (BPA, a model EDC) and E2 allowed a study on the selectivity of MIP particles, whereas the two compounds competed for available binding cavities. Moreover, it was confirmed that addition of E2 after BPA resulted in their occupation of the specific binding sites of MIP particles, displacing the BPA.

In summary, among the three types of synthesized MIP particles, MIP-T7 has a great potential for non-specific removal of EDCs, in addition to specific removal of E2. MIP-T7, with imprinting effect close to 3 and large binding capacity, showed the best binding properties. Investigative characterization of MIP and NIP particles established that CE and DLS could be used for a rapid determination of non-specific binding sites on submicron particles. Last, CE study on binding selectivity of MIP particles led to a better understanding of the binding mechanism between E2 and MIP particles. 


\section{CHAPTER I}

INTRODUCTION 


\subsection{INTRODUCTION}

In an era focused on environmental health, the water industry is faced with the challenge of ensuring a safe supply of drinking water from sustained sources of varying quality [1]. Water contaminants include toxic metals, carcinogenic organic compounds, synthetic chemicals, pharmaceuticals, illicit drugs, cosmetics, personal care products and food supplements, together with their respective metabolites and transformation products [2]. These are of international concern because of their potential to adversely affect human health through consumption of drinking water. More emphasis needs to be placed on endocrine disrupting compounds (EDCs), including estrogens and androgens. The main sources of EDCs in the rivers and lakes of North America and Europe are sewage effluent and agricultural runoff.

Estrogenic compounds are classified as EDCs which interfere with the synthesis, secretion, transport, binding, action or elimination of natural hormones in the body that are responsible for the maintenance of homeostasis, reproduction, development and behaviour [3]. These compounds have become a growing concern due to their potential of harming normal endocrine function and physiological status of humans and animals $[3,4]$. Compounds of this nature have been omnipresent in the environment since the existence of mammals, but it is the growing population of today's society that their effects are starting to be noticed. Both humans and mammals excrete natural estrogens daily. However, conjugated estrogens used in the treatment of cancer, hormonal imbalance, osteoporosis, and other ailments requiring hormonal therapy are also causal to the increase of estrogen pollution [5]. Descriptions of an evident increase in breast and testicular cancers, slow development in infants, and a corresponding decrease in human sperm quantity and quality have raised questions about the role of natural 
estrogens and synthetic estrogenic compounds [6-8]. 17ß-estradiol (E2), 17 $\alpha$-ethynylestradiol (EE2), estrone (E1), and estriol (E3) are four estrogenic compounds contributing to pollution. E2 is the principal intracellular human estrogen and is substantially more active than its metabolites, E1 and E3 [9], their molecular structures and physiochemical properties have been detailed elsewhere [10]. The pharmaceutical EE2 is one of the active ingredients in birth control pills. Synthetic hormones are generally more stable in water than natural hormones and have greater potency $[11,12]$. The current use of pharmacological doses of estrogens $(5-20 \mu \mathrm{g} /$ day in oral contraceptives, $1000 \mu \mathrm{g} / \mathrm{day}$ in hormone replacement therapy) to achieve positive health outcomes is generally recognized as safe.

The main concern that arises from these compounds is their means of entering the aquatic environment from contraceptive residues, hormone replacement therapy residues, and human excretion. Studies in the United Kingdom have shown that the hormones E2, EE2 and E1, although excreted in inactive conjugates, can be degraded in sewage treatment plants to release the active steroid hormones [13]. More concerning is the fact that these estrogens resist degradation during typical sewage treatment operation, exhibit resilience in activated sludge, and destine for release into river waters [14-16]. Sludge from wastewater treatment plants (WWTP) that is used in agricultural fields has a high potential of leaching estrogenic compounds into surface and ground water [17]. Certain control measures have been taken by municipal WWTP to diminish estrogenic compounds in effluents. However, even low concentrations (ng/L) can induce reproductive abnormalities, cause feminization of fish, and decrease the reproduction rate of birds [18-20]. UV radiation, ozonation, membrane filtration, reverse osmosis, and activated carbon adsorption are probable means of improving the removal of estrogens in WWTP [21-24]. Unfortunately, such methods are more costly than they are effective. The foremost challenge is 
that WWTP have to face a complex and variable mixture of numerous organic and inorganic substances. This makes qualitative assignments doubtful and quantitative assays difficult owing to the matrix-induced signal suppression effects or isobaric mass spectral interferences from the complex sample extracts [25]. The reporting limit for estrogens is $5 \mathrm{ng} / \mathrm{L}$ after tertiary treatment (sand filtration, ozonation, and UV disinfection). Relatively, few methods have been developed for the processing of estrogenic pollutants in various environmental samples, especially water, due to their low concentrations present and hence the high sensitivity required. For worse, impoverishment of ecosystems arising from endocrine disturbance will have economic consequences on the efficiency of the food web, which ultimately controls the availability of foodstuffs for humans [1].

The innovation of molecularly imprinted polymers (MIPs) can be utilized for highly selective isolation of specific analytes in sample preparation by solid phase extraction (SPE) [2628]. Syntheses of MIPs are based on the co-polymerization of a functional monomer-analyte (template) complex and a cross-linker. Design variables include the choice of monomer (which complexes with the target analyte at a high binding affinity) [29], selection of a cross-linker (where the length may determine the cavity size), and method of polymerization (which dictates the nature of interactions between polymer matrix and the template to state the ultimate extraction efficiency). The versatility in MIP preparation allows optimization of templatepolymer interactions to model after enzyme-substrate interactions in biochemistry that are highly selective but lack stability. Once the polymer is formed, the template is removed with a proper solvent, leaving a cavity that corresponds with the specific target analyte. In the subsequent process of re-binding for water purification, specific interactions would occur between the smart polymer and the target analyte molecules. In the present work, E2 is chosen as a model EDC 
contaminant for it is considered to be the most physiologically active estrogen $[30,31]$ and has been frequently detected in wastewater [32].

A number of approaches have been proposed for the detection and characterization of submicron particles, including scanning electron microscopy (SEM) [33], dynamic light scattering (DLS) [34], ultrasound spectroscopy [35], and separation techniques such as field flow fractionation (FFF) [36], size exclusion chromatography (SEC) [37], gel electrophoresis (GE) [38], capillary electrophoresis (CE) [39-43], and capillary gel electrophoresis [44]. CE has many advantages over other analytical methods for analyzing submicron particles, including common availability, frit-less operation, high mass sensitivity, high separation efficiency, minute volume of sample, small volume of buffer and measurement of effective mobility, which represents the charge to size ratio $(q / r)$ for a particle [45]. CE has been used to understand the charge of solid lipid nanoparticles in an aqueous suspension [46]. The electrophoretic mobility was measured for $\mathrm{SiO}_{2}$ nanosized particles in a $\mathrm{CE}$ cell at temperatures above $100^{\circ} \mathrm{C}$, and the results indicated a gradual increase in particle electrophoretic response with increasing temperature [47]. Another example of CE analysis of particles was a simple determination of low-density lipoprotein (LDL) particles modified in vitro with $\mathrm{Cu}^{2+}$ and glucose. The LDL electropherograms were highly reproducible with good precisions of effective mobility and peak area [48]. CE for the analysis of colloidal or nano-particles (e.g. polymers and inorganic particles, microbes, mammalian cells, cell subunits and other biological particles) has been recently reviewed [49-51]. In comparison, ion-exchange chromatography, size exclusion chromatography and microchip methods have lower sensitivity and separation efficiency. Most of them are not able to separate structurally similar particles into distinct peaks, and are more laborious and time-consuming. Besides, submicron particles can easily cause damage to the 
injection valve or separation column of chromatographic instruments, as well as different parts of mass spectrometric detectors.

The objective of this study was to characterize MIP and non-imprinted polymer (NIP) particles by a simple CE method, which was a modification of our previous study on quantum dots [52]. The CE method used background electrolyte of medium ionic strength (50 mM sodium borate, $\mathrm{pH} 8.5$ ) to optimize the ionic atmosphere effects around MIP and NIP particles. Consequently these particles were stabilized by the high buffer $\mathrm{pH}$. To the best of our knowledge, this is the first report on characterizing MIP and NIP submicron particles using CE. Meanwhile we can use $\mathrm{CE}$ to accumulate information about the charge state, size, size distribution, dispersion stability, and surface binding sites of the particles. Moreover, this simple CE method is unique in being able to separate the different kinds of particles that are migrating at different mobilities. It also opens up new potential to investigate their thermodynamics and kinetics for binding EDCs. Meanwhile binding properties of MIP and NIP particles were appraised using HPLC as a reference method for validating CE as a new method for the determination of EDCs without prior removal of the particles.

\subsection{Principal aspects of molecular imprinting}

Molecular imprinting is a powerful technique based on creating recognition sites, in a synthetic polymer matrix, that are complementary to the size, shape and chemical functionality of the template molecule. A molecular imprinted polymer can be formed by radical polymerization of a functional monomer in the presence of a template that has complementary functional groups. The template is extracted afterwards, thus leaving behind complementary cavities with chemical affinity for the original template molecule. These cavities exhibit mechanistic functionality 
similar to those of antibodies or enzymes. The technique of molecular imprinting has been applied with success to the preparation of materials with high affinity for separations [53,54], binding assays [55], sensor science [56-58], and catalysis [59], wherever a highly specific interaction of the target molecule (analyte) with the MIPs is demanded. A wide variety of MIPs have been screened to meet the present demand of higher selectivity in various fields, such as environmental control [60], drug delivery [61], health protection [62], forensic science [63], and biotechnology [64].

Synthesizing the MIP particles requires a great attention on choosing numbers of variables such as template, functional monomer, cross-linker, initiator, porogen, temperature and methodology of polymerization.

\subsubsection{Template}

A MIP usually synthesized for a specific analytical use that implies the choice of a given template molecule and that's how it is in center of importance [65]. Templates direct the organizations of the functional monomer around themselves and all compounds cannot be used as a template. The template must have functional groups that can interact with the functional group of the functional monomers. Their functional group must not inhibit the polymerizations process. They have to be stable in polymerization situation and through extraction procedure [66].

\subsubsection{Functional monomers}

The functional monomers are responsible for the binding on MIP particles. Usually they are used in excess in comparison with a template molecule to obtain suitable equilibrium 
concentration of template monomer construction. To create a strong template-monomer interaction a complementary functionality on the functional monomers is essential. As of most favorable functional monomer families, we can point to the carboxylic-acid based monomers. These monomers can interact with a template molecule in various ways (both hydrogen bonding donor and acceptance), as they possess only moderate rotational degrees of freedom. Methacrylic acid (MAA) as one of the most used functional monomer has a methyl group that comparing to acrylic acid restricts the conformational freedom and provides additional van der waals interaction, which defines the shape selective elements of receptor sites. Structure of MAA and acrylic acid are shown in Figure 1.
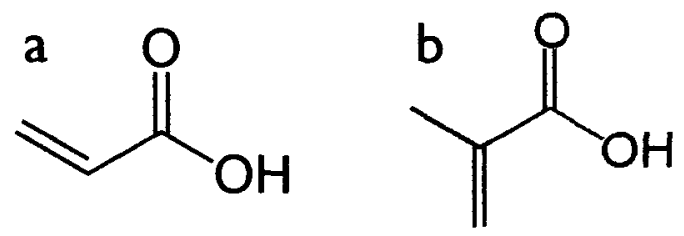

Figure 1 Structures of a) acrylic acid, b) methacrylic acid.

\subsubsection{Cross-linkers}

Cross linkers are carrying the most important role in the MIP particles. Cross-linkers control the morphology and stability of the polymer matrix. In addition, cross-linkers stabilize the imprinted binding site and maintain the three dimensional memory of the template. The MIP polymer should be flexible enough to allow the diffusion of the templates. However, for keeping the cavity of the template molecule, optimum hardness of the polymer is essential [67]. A large variety of cross-linkers are compatible with the molecular imprinting manner. Ethyleneglycole dimethacrylate (EGDMA) is one of the most common cross-linker that is used in preparation of 
either bulk or MIP particles. As seen in structure of EGDMA, a spacer links two methacrylate moieties. Most often di-functional cross-linkers have been used, but in some cases tri-functional cross-linker such as trimethylol propane trimethacrylate (TRIM) is used, while reported to deliver a better performance [68]. Chemical structure of E2, bisphenol A (BPA), EGDMA and TRIM are shown in Figure 2.

a<smiles>CC12CCC3c4ccc(O)cc4CCC3C1CCC2O</smiles>

C<smiles>C=C(C)C(=O)OCCOC(=O)C(=C)C</smiles><smiles>CC(C)(c1ccc(O)cc1)c1ccc(O)cc1</smiles>

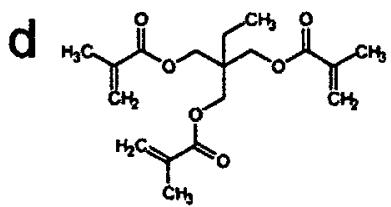

Figure 2 Structures of a) $17 \beta$-estradiol, b) bisphenol A, c) ethyleneglycol dimethacrylate and d) trimethylolpropane trimethacrylate.

\subsubsection{Porogens}

Porogens are the solvent of used in the polymerization, which are responsible to dissolve all the templates, functional monomers and cross-linkers in the same phase. Besides, the polymerization solvents create the pores in the polymer reaching a macro-porous morphology. For all above mentioned reasons, the solvent used in MIP preparations is called porogens. In general increasing the amount of porogens results in large pores. 


\subsubsection{Initiators}

In most cases MIP materials are polymerized by a free-radical mechanism. Depending to the nature of the functional monomers and the templates the polymerization mixture can be initiated either thermally or through a photochemical reaction. $2,2^{\prime}$-azobisisobutyronitrile (AIBN) is the most commonly used initiator for methacrylate-base MIP materials. AIBN can be decomposed in either low temperature by UV radiation or thermally at about $60^{\circ} \mathrm{C} \mathrm{[53].} \mathrm{The} \mathrm{preparation} \mathrm{of}$ free radical in AIBN followed by polymerization of MAA shown in Figure 3.
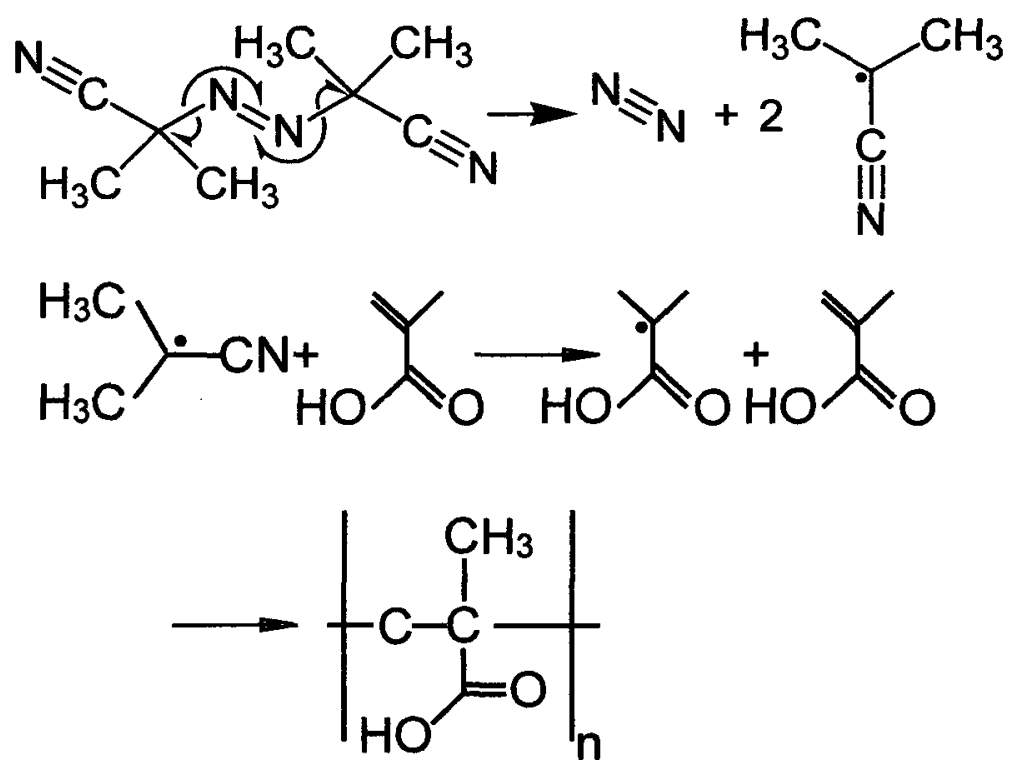

Figure 3 Polymerization of MAA after decomposition of AIBN to produce a free radical.

\subsubsection{Template-monomer interactions}

There are different approaches regarding the interaction between the template and the functional monomer. As the First one, in covalent approach, the polymerization of template and the functional monomer in the presence of cross-linker results in the formation of covalent bond 
between template and functional monomer. So both removal and binding on templates involves a chemical cleavage of the bound templates. In addition, the functional groups in the product are always in associate with template sites, which results in smaller non-specific binding sites and better imprinting effect. However, the numbers of the templates that adapt to this approach are very limited. In the second case as non-covalent approach, template and functional monomers interact weakly through $\mathrm{H}$-Bonding, ion pairing, dipole-dipole and van der waals interactions [69]. Based on simplicity of this method, it's widely used. There is no need for preparation of template-monomer adduct before polymerization. This method can be adopted for a large variety of the templates. Figure 4 represents a schematic preparation of MIP particles using noncovalent approach.

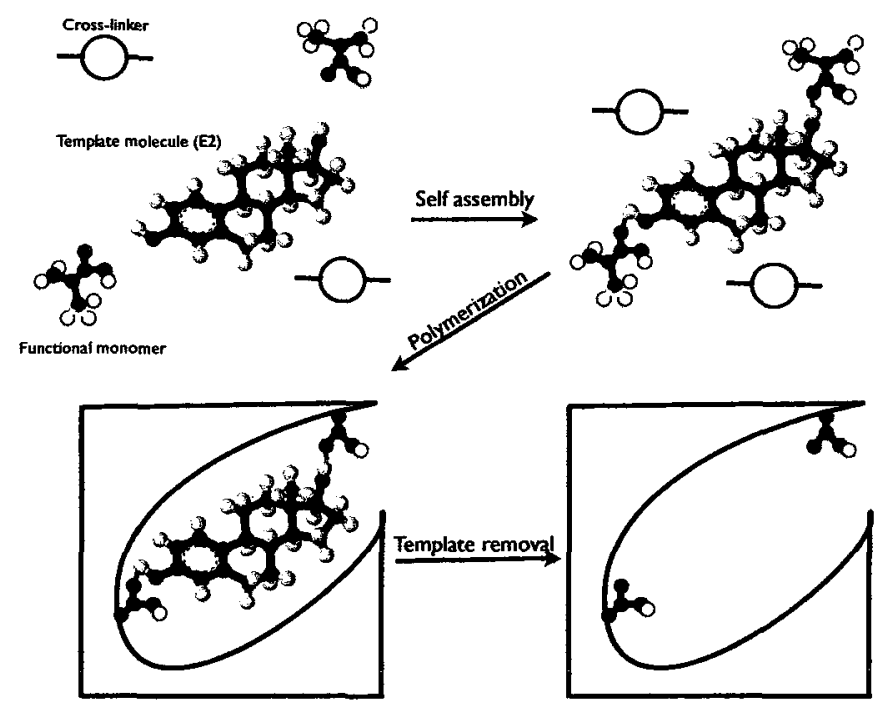

Figure 4 Schematic preparation of MIP particles.

\subsection{Capillary electrophoresis}

The electrophoresis is the movement of sample ions or any charged species under the influence of an applied voltage. The ion will move towards the appropriate electrode and pass through a 
detector. The principal components are the high-voltage power supply, a capillary that passes through the optical center of the detection system. The capillary is first filled with the required buffer solution. Sample solution (typically $1-20 \mathrm{~nL}$ ) is then introduced at the end of the capillary away from the detector, which in normal polarity is close to the anode. Both ends of capillary then dipped into reservoirs containing high-voltage electrodes and the buffer solution. Electrodes are composed of an inert material, such as platinum. Applying of voltage (typically 10-30 kV) across the capillary causes electrphoretic and electroosmotic movement, resulting in the charged species in the sample moving along the capillary and passing through the online detector. A typical instrument setup is shown in Figure 5.
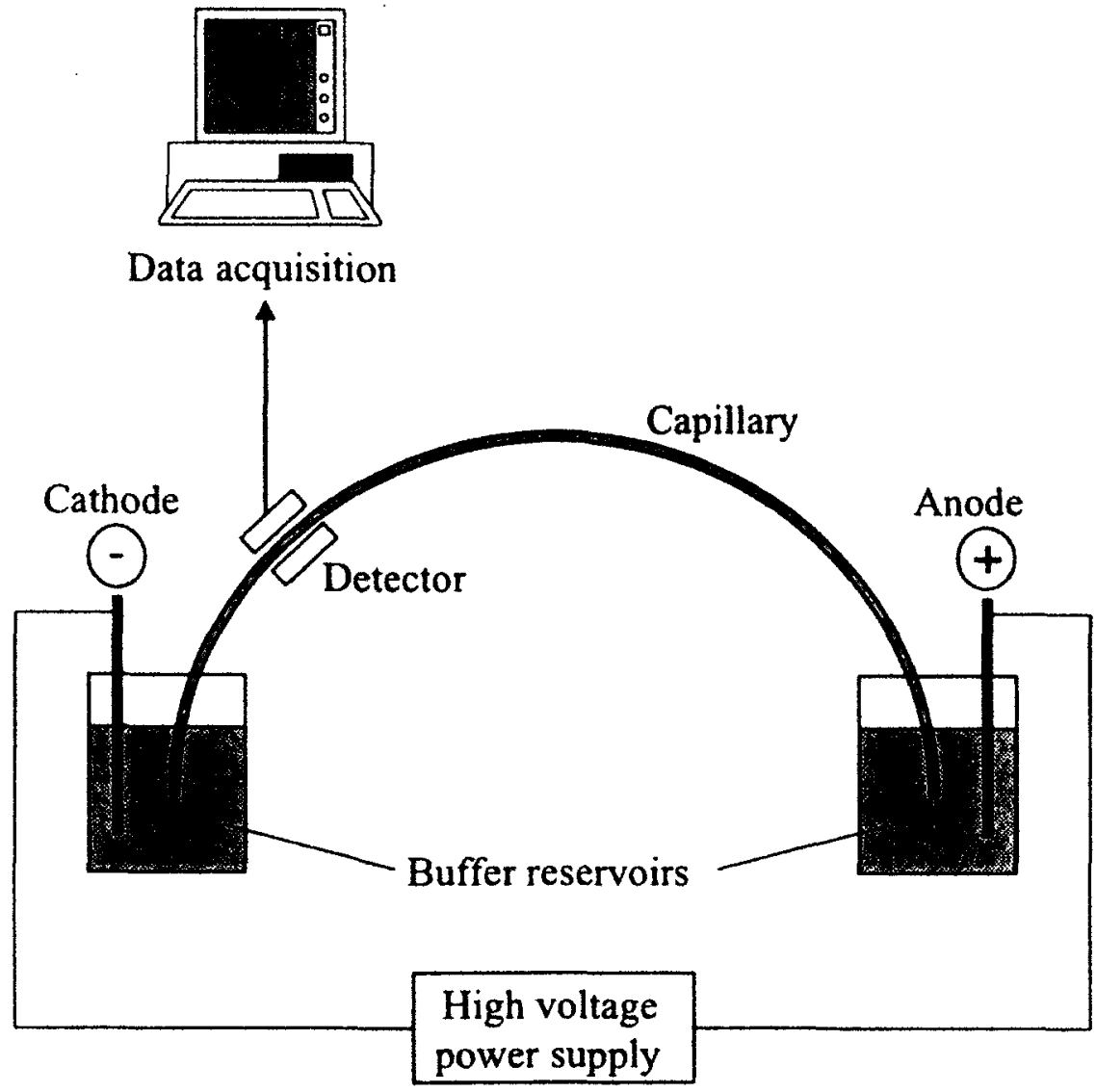

Figure 5 Typical instrumental set up of capillary electrophoresis. 


\subsubsection{Electophoretic mobility}

When an electric field (E) is applied on a charged ion/species (q) the force on the species will be equal to $\mathrm{qE}$. In the other side in the solution the retarding frictional force is f.u $\mathrm{u}_{\mathrm{ep}}$, where $\mathrm{u}_{\mathrm{ep}}$ is the electrophoretic velocity of the charged species and $f$ is the frictional coefficient. Then as accelerating force equals to the frictional force, the species quickly reaches to the steady speed.

$\mathrm{qE}=\mathrm{f} \mathrm{u}_{\mathrm{ep}}$

$\therefore \mathrm{u}_{\mathrm{ep}}=(\mathrm{q} / \mathrm{f}) \mathrm{E}=\mu_{\mathrm{ep}} \mathrm{E}$

Equation 1

Electrophoretic mobility $\left(\mu_{\mathrm{ep}}\right)$ is a constant of proportionality between the speed of the charged species and the strength of electric field.

\subsubsection{Electroosmotic flow}

The inner capillary walls are covered with silanol (Si-OH), which in any $\mathrm{pH}$ higher than $\sim 2$ are negatively charged ( $\left.\mathrm{Si}^{-} \mathrm{O}^{-}\right)$. The fixed negative charged on the inner wall and the excess cation near the wall results on a double layer (Figure 6). A tightly adsorbed layer of the cations neutralizes partially the walls negative charges and the remaining negative charges of capillary is neutralized by mobile cations that are in diffuse part of the double layer in the solution near the wall.

In an electric field, cations are attracted towards the cathode while anions are pulled towards the anode. The excess cations in the diffuse part of double layer instruct the net momentum towards the cathode, which is called electrophoresis. The constant of proportionality between electroosmotic velocity $\left(\mathrm{u}_{\mathrm{eo}}\right)$ and applied electric field is called electroosmotic mobility $\left(\mu_{\mathrm{eo}}\right)$.

$\mathrm{u}_{\mathrm{eo}}=\mu_{\mathrm{eo}} \mathrm{E}$

Equation 2

Fixed negative charge of inner wall 


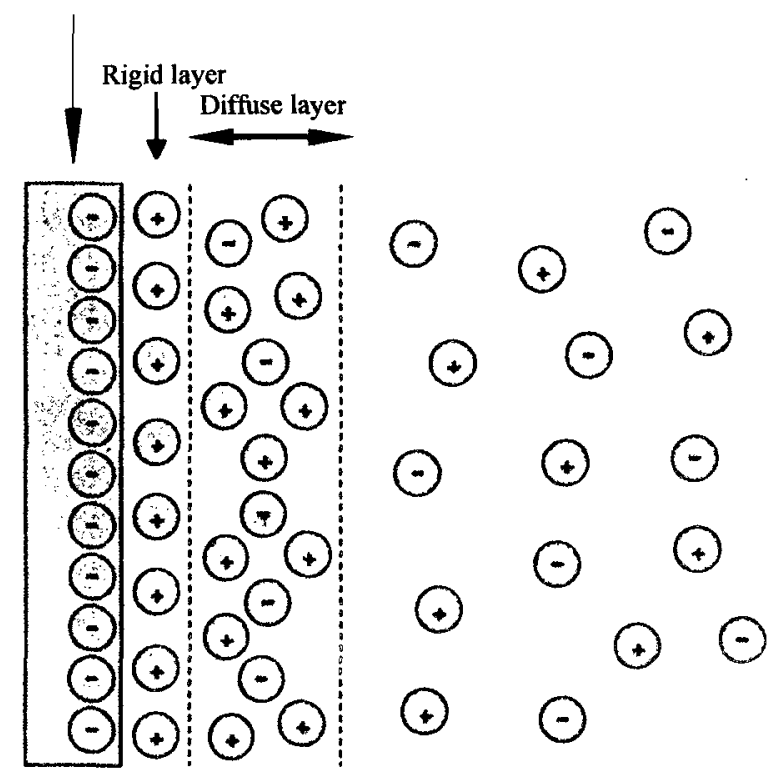

Frign

\subsubsection{Apparent mobility}

Mobility or apparent mobility of a species is known as the summation of electrophoretic mobility of the species and electroosmotic mobility of the solution.

$\mathrm{M}_{\mathrm{app}}=\mu_{\mathrm{ep}}+\mu_{\mathrm{eo}}$

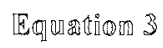

The apparent mobility of a species is the net speed of the species divided by the electric field.

$M_{a p p}=u_{n e t} / E=\left(L_{d} / t\right) /\left(V / L_{t}\right)$

Where $L_{d}$ : length of capillary to the detector

$$
\begin{aligned}
& L_{t} \text { : Total length of capillary } \\
& t \text { : migration time }
\end{aligned}
$$

Electroosmotic flow of is calculated by adding a neutral solute that is detectable by the CE detector, and then its migration time ( $\left.\mathrm{t}_{\text {neutral }}\right)$ is measured.

$M_{e o}=u_{\text {neutral }} / E=\left(L_{d} / t_{\text {neutral }}\right) /\left(V / L_{t}\right)$ 
Then the electrophoretic mobility of an analyte can be calculated by subtracting the electroosmotic mobility of solution from the apparent mobility of the analyte.

$\mathrm{M}_{\mathrm{ep}}=\mu_{\mathrm{app}}-\mu_{\mathrm{eo}}$

Equation 6 


\section{CHAPTER II}

\section{EXPERIMENTAL}




\subsection{Synthesis and properties of MIP and NIP particles}

\subsubsection{Preparation of MIP and NIP submicron particles}

\subsubsection{Reagents}

17ß-Estradiol (E2) and Triethylamine (TEA) were purchased from Sigma (St. Louis, MO, USA); acetonitrile was HPLC-grade solvent obtained from Fisher (Ottawa, ON, Canada); 2,2'azobisisobutyronitrile (AIBN) was bought from Pfaltz \& Bauer (Waterbury, CT, USA); acetone was spectro grade solvent obtained from Caledon (Georgetown, ON, Canada); methacrylic acid (MAA), ethylene glycol dimethacrylate (EGDMA) and trimethylolpropane trimethacrylate (TRIM) were purchased from Aldrich (St. Louis, MO, USA).

\subsubsection{Instrumentation}

HPLC analysis were carried out using a system with a solvent pump (Shimadzu LC-6A, Kyoto, Japan), injector valve (Valco Cheminert VIGI C2XL, Houston, TX, USA) equipped with a sample loop $(20 \mu \mathrm{L})$, column (Keystone Scientific Nucleosil C18, $3 \mu \mathrm{m}, 150 \times 2 \mathrm{~mm}$, State College, Pennsylvania, USA), fluorescence detector (Perkin-Elmer LC 240, Boston, MA, USA), and data acquisition system (Baseline Chromatography N2000, Tianjin, China).

Particles were imaged using a tungsten-filament, variable pressure SEM, (Tescan Vega II, XMU, Cranberry Twp., PA) at an accelerating voltage of 10-20 kV. The dynamic diameters of particles were measured by a particle size analyzer (Brookhaven Instruments nano-DLS, Holtsville, NY, USA) based on DLS. 


\subsubsection{Polymerization Method}

The initial procedure for synthesis of MIP particles (MIP-E) was based on a previously established method [70], besides different MIPs using another cross-linker (TRIM) and various mole ratios between functional monomer and cross-linker were prepared as listed in Table 1 .

The template molecule, E2, was added to a mixture of porogen solvent (acetone/acetonitrile, 1:3 v/v) and functional monomer (MAA). After mixtures were ultra sonicated for 3 min, cross-linker (either EGDMA or TRIM) was added. The initiator (AIBN) was next added to the mixture. After 5 min of sonication followed by $5 \mathrm{~min}$ of degassing by purging with nitrogen, the solution was sealed and left in a hot water bath at $60^{\circ} \mathrm{C}$ for 24 hours. Non-imprinted polymer (NIP) particles were prepared similarly, in the absence of the template molecules.

Table 1 Composition of pre-polymerization mixture for the synthesis of molecularly imprinted polymers, specifically for E2.

\begin{tabular}{lllll}
\hline Polymer & Template molecule & $\begin{array}{l}\text { Functional } \\
\text { monomer }\end{array}$ & Cross-linker & Mole ratio \\
\hline MIP-E & E2 & MAA & EGDMA & $1: 8: 6.7$ \\
NIP-E & - & MAA & EGDMA & $0: 8: 6.7$ \\
MIP-T4 & E2 & MAA & TRIM & $1: 8: 4$ \\
NIP-T4 & - & MAA & TRIM & $0: 8: 4$ \\
MIP-T7 & E2 & MAA & TRIM & $1: 8: 7$ \\
NIP-T7 & - & MAA & TRIM & $0: 8: 7$ \\
\hline
\end{tabular}




\subsubsection{Template bleeding}

$5.0 \mathrm{mg}$ of MIP-T4 and MIP-T7 particles (before removal of the template) were placed in six 1.5$\mathrm{mL}$ centrifuge micro tubes. $1 \mathrm{~mL}$ of six different concentrations of E2 $(0.0-1.0 \mu \mathrm{g} / \mathrm{mL})$ was added to each of the micro tubes. After 30 seconds of sonication, the homogenous mixtures were incubated for 2 minutes. Then they were centrifuged, and the supernatant were collected for analytical determination of E2 using HPLC-FD [60]. The mobile phase (1:1:2 v/v acetonitrile/methanol/water) was pumped at a flow rate of $0.4 \mathrm{~mL} / \mathrm{min}$. Fluorescence detection of E2 was performed at optimal excitation and emission wavelengths of $220 \mathrm{~nm}$ and $310 \mathrm{~nm}$.

\subsubsection{Template removal}

Polymerization products were centrifuged at $10,000 \mathrm{rpm}$ for $10 \mathrm{~min}$ and supernatant discarded. Both MIP and NIP particles were rinsed 3 times with an aliquot of $5 \mathrm{~mL}$ acetonitrile and then samples were dried in the oven at $50^{\circ} \mathrm{C}$ for overnight. To remove the template, $100 \mathrm{mg}$ of dry MIP particles were placed into a $2-\mathrm{mL}$ micro tube and $1 \mathrm{~mL}$ of $5 \%$ triethylamine (TEA) in methanol was added on to the sample. After sonication for 5 min the obtained homogenous suspension was centrifuged for $5 \mathrm{~min}$ and supernatants were discarded. MIP particles were washed 6-8 times and after the fifth wash the supernatants were collected for HPLC-FD analysis as a control for template removal. After the removal of template was succeeded the MIP particles were rinsed three times with an aliquot of $1 \mathrm{~mL}$ acetonitrile for removal of potential TEA remained in the particles, residing in cavities and pores. At last, after drying the particles in the oven, they manually crushed as they clump together during drying process. 


\subsubsection{MIP and NIP particles morphology and size}

Different types of MIP and NIP particles were deposited onto individual carbon tape cartridges, and coated with less than $5 \mathrm{~nm}$ of gold using a RF sputter (Anatech Hummer, Union City, CA, USA). Particles were imaged using a tungsten-filament, variable pressure SEM, (Tescan Vega II, XMU, Cranberry Twp., PA, USA) with an accelerating voltage of $10-20 \mathrm{kV}$. The dynamic diameters of particles were measured, based on DLS, by a particle size analyzer (Brookhaven Instrument nano-DLS, Holtsville, NY, USA). For accuracy, 7 different aliquots of $0.01 \mu \mathrm{g} / \mathrm{mL}$ particles were analyzed and the dynamic diameter of each aliquot was measured in 10 replicates of 30 seconds.

\subsubsection{Batch binding experiments}

Binding capacities and efficiencies of the different types of MIP and NIP particles were evaluated. Also the minimal amount of particles for best binding efficiencies was determined through the following experiments.

\subsubsection{Binding capacity}

Different aqueous solutions of E2 varying from $1-10 \mu \mathrm{g} / \mathrm{mL}$ were prepared in DDW. A 2-mL aliquot of each solution was added to $0.5 \mathrm{mg}$ of different MIP and NIP particles. After 1 minute of sonication, the homogenous mixtures were incubated for 15 hours. Then they were centrifuged, and the supernatant were collected for analytical determination of remaining E2 $\left(\mathrm{C}_{\mathrm{f}}\right)$ using HPLC-FD. The amounts of bound E2 (n) were calculated by subtracting $\mathrm{C}_{\mathrm{f}}$ from the initial E2 concentrations. Then the ratios of bound E2 to the remaining E2 $\left(n / C_{f}\right)$ versus bound E2 (n) 
were plotted, and the association constant and binding capacity were determined from the slope and intercept of the plot respectively.

\subsubsection{Binding isotherm}

Different amount of MIP particles $(0.2-20 \mathrm{mg}$ ) were placed in $1.5-\mathrm{mL}$ polypropylene centrifuge micro tubes. An aliquot of $1 \mathrm{~mL}$ aqueous E2 solution $(0.1 \mu \mathrm{g} / \mathrm{mL})$ was added to each micro tube. To obtain a homogenous suspension the mixture was sonicated for $30 \mathrm{~s}$. After incubation for 2 min, the mixture was centrifuged at $10,000 \mathrm{rpm}$ for $1 \mathrm{~min}$. Concentrations of remaining E2 in the supernatant were measured by HPLC-FD analysis. The amounts of remaining E2 after binding on different types of MIP particles were calculated. The experimentally obtained data were plotted versus masses of MIP particles.

\subsubsection{Binding efficiency}

A $20.0 \mathrm{mg}$ of MIP particles cross-linked by EGDMA (MIP-E) were placed in 1.5-mL polypropylene centrifuge microtubes. An aliquot of $1 \mathrm{~mL}$ of aqueous E2 solution (0.1-1.0 $\mu \mathrm{g} / \mathrm{mL}$ ) was added to each microtube. The mixture was sonicated for $30 \mathrm{~s}$ to obtain a homogenous suspension. After incubation for $2 \mathrm{~min}, 15 \mathrm{~min}$ or $1 \mathrm{hr}$, the mixture was centrifuged at $10,000 \mathrm{rpm}$ for $1 \mathrm{~min}$. The supernatant was collected for HPLC-FD analysis to determine the concentration of remaining E2. The related peak areas of remaining E2 were plotted versus initial E2 concentration in comparison with areas of E2 before binding on MIP particles to visualizing the binding properties of particles on E2. Binding \% of E2 on particles was calculated using (Eq. 7). Binding \% of E2 on MIP-E after 2 and 15 minutes were plotted for comparison to each other. 
\%Binding $=100(\mathrm{n} /$ initial [E2])

Equation 7

Where $\mathrm{n}=$ Bound $[\mathrm{E} 2]$

As the preliminary results, showed a great efficiency after 2 min incubation, same experiments were carried out using $0.5 \mathrm{mg}$ of MIP-E.

In the other experiment, binding efficiencies of different MIP and NIP particles, crosslinked by various ratios of TRIM (MIP-T4 and MIP-T7) were deliberate. The 0.5 and $5.0 \mathrm{mg}$ of particles was placed in series of microtubes and a $1 \mathrm{~mL}$ volume of different concentrations of E2 was added to each vial. Then after 2 minutes of the incubation vials were centrifuged at 10,000 $\mathrm{rpm}$ and supernatant were tested for remaining E2. At the end, binding efficiencies of particles were plotted versus the initial concentrations of E2.

\subsubsection{Leakage of bound E2}

A $5.0 \mathrm{mg}$ of MIP-T4 or MIP-T7 was placed in 1.5-mL microtubes. An aliquot of $1 \mathrm{~mL}$ various concentrations of E2 $(0.2-10 \mu \mathrm{g} / \mathrm{mL})$ was added to each vial. The mixtures were sonicated for 30 seconds. After 5 minutes of incubation, the supernatant samples were analyzed by HPLC-FD for determination of remaining E2. Bound E2 were determined by subtracting the remaining E2 from initial concentration of E2. Next, $1 \mathrm{~mL}$ of pure water was added to each vial containing MIP-T4 or MIP-T7 particles. The mixtures were incubated for 2 and 6 hours. After sonication, the supernatants were analyzed by HPLC-FD for determination of any leaking E2. 


\subsection{Capillary Electrophoresis for characterization of MIP and NIP particles}

\subsubsection{Separation of MIP and NIP particles using CE}

Individual $1.0-\mathrm{mg} / \mathrm{mL}$ mixtures of different types of MIP or corresponding NIP particles in the CE buffer solution were prepared. The mixtures were sonicated for $30 \mathrm{~s}$ to obtain a homogenous sample. After preconditioning the $\mathrm{CE}$ instrument, individual mixture was injected for 1 second by introducing 0.5 -psi nitrogen gas on the surface of the vial-containing sample (hydrodynamic injection). Besides, to introduce the mixture of MIP and corresponding NIP particles, a 2.0 $\mathrm{mg} / \mathrm{mL}$ of MIP and NIP particles in buffer solution were prepared and after sonication for 30 seconds, a 1:1 (v/v) mixture of MIP and NIP particles were introduced to CE instrument for further separation.

\subsubsection{Reagents}

Boric acid was purchased from Fisher Scientific (Ottawa, ON, Canada); BPA and mesityle oxide were purchased from Sigma (St. Louis, MO, USA); EOTrol ${ }^{\mathrm{TM}}(3 \% \mathrm{w} / \mathrm{v})$ dynamic coating was obtained from Target Discovery (Palo Alto, CA, USA). For preparation of all solutions, distilled deionized water (DDW) (18 M $2 . \mathrm{cm})$ was used. Different concentration of borate buffers were prepared by dissolving an appropriate amount of boric acid, then $\mathrm{pH}$ was adjusted using 0.1 or 1 $\mathrm{M} \mathrm{NaOH}$ solutions, and the mixture was diluted to $100 \mathrm{~mL}$ in a volumetric flask. A background electrolyte (BGE) containing $0.005 \% \mathrm{w} / \mathrm{v}$ EOTrol ${ }^{\mathrm{TM}} \mathrm{LN}$ was prepared by dissolving $25 \mu \mathrm{L}$ of EOTrol ${ }^{\mathrm{TM}} \mathrm{LN}(3 \% \mathrm{w} / \mathrm{v})$ in $15 \mathrm{~mL}$ of $50 \mathrm{mM}$ borate buffer $(\mathrm{pH} 8.5)$. All prepared BGE was filtered through an Acrodisc $®$ PSF syringe filter with $0.2 \mu \mathrm{m}$ porosity Supor ${ }^{\circledR}$ membrane (Pall PES, East Hills, NY, USA). 


\subsubsection{Instrumentation}

All CE experiments were performed using a Beckman P/ACE System 2100 (Fullerton, CA, USA), which was equipped with a liquid-cooling system and a UV detector. The operation system was controlled by a personal computer through the Gold software. The Polymicro Technologies (Phoenix, Az. USA) capillaries with inner and outer diameters of 100 and $364 \mu \mathrm{m}$ respectively, and a total length of $68 \mathrm{~cm}(61 \mathrm{~cm}$ to the detector) were used. The temperature of the capillary cartridge was set at $25.0 \pm 0.1^{\circ} \mathrm{C}$ for all experiments.

\subsubsection{Capillary treatment procedure}

A previously developed method was applied for preconditioning of capillary before initial use and daily use [71]. Prior to initial use, the capillary was sequentially rinsed with methanol (5 $\min )$, water (2 min), $1 \mathrm{M} \mathrm{HCl}(5 \mathrm{~min})$, water $(2 \mathrm{~min}), 1 \mathrm{M} \mathrm{NaOH}(10 \mathrm{~min})$, water $(2 \mathrm{~min})$ and BGE (20 min). At the beginning of each day, the capillary was washed with $1 \mathrm{M} \mathrm{NaOH}(5 \mathrm{~min})$, water ( $2 \mathrm{~min}), \mathrm{BGE}(20 \mathrm{~min})$ and finally it was electro-conditioned by applying a voltage of 30 $\mathrm{kV}$ for $15 \mathrm{~min}$. Before injection of each sample, the capillary was rinsed with $0.1 \mathrm{M} \mathrm{NaOH} \mathrm{(1}$ $\mathrm{min})$, water (1 min) and BGE (2 min). At the end of the day, a final washing with $1 \mathrm{M} \mathrm{NaOH}(1$ $\min$ ) followed by water $(1 \mathrm{~min})$ was performed while both ends of capillary were left in the DDW vial for the night. In case of leaving capillary for a longer period of time after rinsing with DDW, capillary were rinsed with methanol and then nitrogen gas were blown in the capillary in the reverse direction for a minute and then capillary were stored for the next run. 


\subsubsection{Addition of capillary modifier and separation of MIP and NIP particles}

The individual $1.0-\mathrm{mg} / \mathrm{mL}$ mixture of different types of MIP or corresponding NIP particles in the CE buffer solution in the absence of any modifier were prepared $(50 \mathrm{mM}$ borate buffer $\mathrm{pH}=8.5$ ). The mixtures were sonicated for $30 \mathrm{~s}$ to obtain a homogenous sample. After preconditioning the $\mathrm{CE}$ instrument individual mixture was injected for 1 second by introducing 0.5-psi nitrogen gas on the surface of the vial-containing sample (hydrodynamic injection). Besides, to introduce the mixture of MIP and corresponding NIP particles, a $2.0 \mathrm{mg} / \mathrm{mL}$ of MIP and NIP particles in buffer solution were prepared and after sonication for 30 seconds, a 1:1 (v/v) mixture of MIP and NIP particles were introduced to CE instrument for further separation. The CE electropherograms of individual MIP or NIP and the mixtures of MIP and NIP particles were obtained by using $50 \mathrm{mM}$ borate buffer $\mathrm{pH}=8.5$, containing $0.005 \% \mathrm{EOTroL}^{\mathrm{TM}} \mathrm{LN}$ and detected at $214 \mathrm{~nm}$.

\subsubsection{Kinetic study of NIP particles binding with bisphenol A}

A suspension of $2.0 \mathrm{mg} / \mathrm{mL}$ of NIP-T4 or NIP-T7 particles in BGE (50 mM borate buffer, $\mathrm{pH}$ 9.2) was prepared and sonicated for $30 \mathrm{~s}$ to obtain homogeneity. A $40-\mu \mathrm{g} / \mathrm{mL}$ solution of BPA in BGE was also prepared. To obtain a mixture of $1 \mathrm{mg} / \mathrm{mL}$ of particles in $20 \mu \mathrm{g} / \mathrm{mL}$ BPA, a 1:1 mixture of the particle suspension and $40-\mu \mathrm{g} / \mathrm{mL}$ BPA solutions was vortexed efficiently at the maximum speed for $5 \mathrm{~s}$. After incubation for 20-120 s, the mixture was injected immediately for $\mathrm{CE}$ analysis to determine the concentration of remaining BPA after non-specific binding with NIP particles. 


\subsubsection{Selectivity of particles using $\mathrm{CE}$}

\subsubsection{Selectivity of MIP-T4 particles on E2 in presence of BPA}

The suspension of $2.0 \mathrm{mg} / \mathrm{mL}$ MIP-T4 or NIP-T4 particles in BGE ( $50 \mathrm{mM}$ borate buffer, $\mathrm{pH}$ 9.2) were prepared. Then the mixtures were sonicated for $30 \mathrm{~s}$ to obtain homogeneity. A fresh mixture of $1.0 \mathrm{mg} / \mathrm{mL}$ of particles in the solutions of $20 \mu \mathrm{g} / \mathrm{mL}$ BPA containing a variety of E2 solutions $(0,1,2,5,8$ and $12 \mu \mathrm{g} / \mathrm{mL})$ were prepared. After efficiently mixing, using vortex for 5 seconds, mixtures were introduced to the $\mathrm{CE}$ through a $9 \mathrm{~s}$ electrokinetic injection at $10 \mathrm{kV}$. In the other set of the experiment, mixture of particles and various concentration of BPA, which were equal to summation of concentrations of BPA and $\mathrm{E} 2,(20,21,22,25,28$ and $32 \mu \mathrm{g} / \mathrm{mL})$ were prepared. Particles were incubated into the solution for $20 \mathrm{~s}$ while the time were monitored and controlled. Separation voltage of $20 \mathrm{kV}$ was applied and the BPA was detected at $214 \mathrm{~nm}$. The $\mathrm{CE}$ peak areas of the BPA in the characteristic migration time were plotted versus concentration of added E2.

\subsubsection{Selectivity of MIP-T7 particles on E2 in presence of BPA}

The same experiments as 2.2.4.1 were repeated on MIP-T7 and NIP-T7 for comparison of their selectivity characteristics.

\subsubsection{Non-specific binding of BPA on MIP-T4 and MIP-T7 after binding with E2}

The suspension of $2.0 \mathrm{mg} / \mathrm{mL}$, MIP-T4 or MIP-T7 particles in BGE ( $50 \mathrm{mM}$ borate buffer, $\mathrm{pH}$ 9.2) were prepared and sonicated for $30 \mathrm{~s}$ to attain a homogeneous mixture. Then to obtain the final required concentration of $\mathrm{E} 2,10 \mu \mathrm{L}$ of various concentrations of $\mathrm{E} 2$ in methanol were added 
to the particles. Next, after incubation of the mixture for $20 \mathrm{~s}, 260 \mu \mathrm{L}$ of the $40 \mu \mathrm{g} / \mathrm{mL}$ BPA was added to the mixture of MIP particles and E2. Finally, after $20 \mathrm{~s}$ of incubation, mixture was introduced to the CE. All above steps are summarized in the Table 2.

Table 2 Sample preparation for characterization of binding mechanisms of MIP particles.

\begin{tabular}{|c|c|c|c|c|c|}
\hline & & \multirow{3}{*}{$\begin{array}{l}\text { Step } 1 \\
V \text { of } 2 \mathrm{mg} / \mathrm{mL} \text { particles } \\
(\mu L)\end{array}$} & \multicolumn{2}{|l|}{ Step 2} & \multirow{3}{*}{$\begin{array}{l}\text { Step } 3 \\
V \text { of }(40 \mu \mathrm{g} / \mathrm{mL}) \mathrm{BPA}\end{array}$} \\
\hline \multicolumn{2}{|c|}{$\begin{array}{l}\text { Relative concentration to the } \\
1 \mathrm{mg} / \mathrm{mL} \text { of particles }\end{array}$} & & \multicolumn{2}{|c|}{ Added E2 } & \\
\hline E2 $(\mu \mathrm{g} / \mathrm{mL})$ & BPA $(\mu \mathrm{g} / \mathrm{mL})$ & & $V(\mu L)$ & $C(\mu \mathrm{g} / \mathrm{mL})$ & \\
\hline$\overline{0}$ & 20 & 260 & 0 & & 260 \\
\hline 1 & 20 & 250 & 10 & 25 & 260 \\
\hline 2 & 20 & 250 & 10 & 50 & 260 \\
\hline 4 & 20 & 250 & 10 & 100 & 260 \\
\hline 5 & 20 & 250 & 10 & 125 & 260 \\
\hline 6 & 20 & 250 & 10 & 150 & 260 \\
\hline 8 & 20 & 250 & 10 & 200 & 260 \\
\hline 10 & 20 & 250 & 10 & 250 & 260 \\
\hline 12 & 20 & 250 & 10 & 300 & 260 \\
\hline
\end{tabular}

\subsubsection{Binding competition of E2 and BPA on MIP-T4 and MIP-T7 particles}

The suspension of $2.0 \mathrm{mg} / \mathrm{mL}$, MIP-T4 or MIP-T7 particles in BGE ( $50 \mathrm{mM}$ borate buffer, $\mathrm{pH}$ 9.2) were prepared and sonicated for $30 \mathrm{~s}$ to obtain a homogeneous mixture. Then $250 \mu \mathrm{L}$ of suspended particles were placed into the $2-\mathrm{mL}$ glass vial and $250 \mu \mathrm{L}$ of $40 \mu \mathrm{g} / \mathrm{mL}$ BPA was added into the vial. Mixtures were vortexed for $5 \mathrm{~s}$ in maximum speed. After incubation for 20 seconds, $10 \mu \mathrm{L}$ of different concentration of E2 in methanol (as presented in Table 3) were added. Next, after another 20 seconds of incubation mixture was introduced to the $\mathrm{CE}$ through 9 seconds of electrokinetic injection at $10 \mathrm{kV}$. CE electropherograms were obtained, using the same BGE by applying separation voltage of $20 \mathrm{kV}$ and detected at $214 \mathrm{~nm}$. 
Table 3 Sample preparation for binding competition of E2 and BPA on MIP particles.

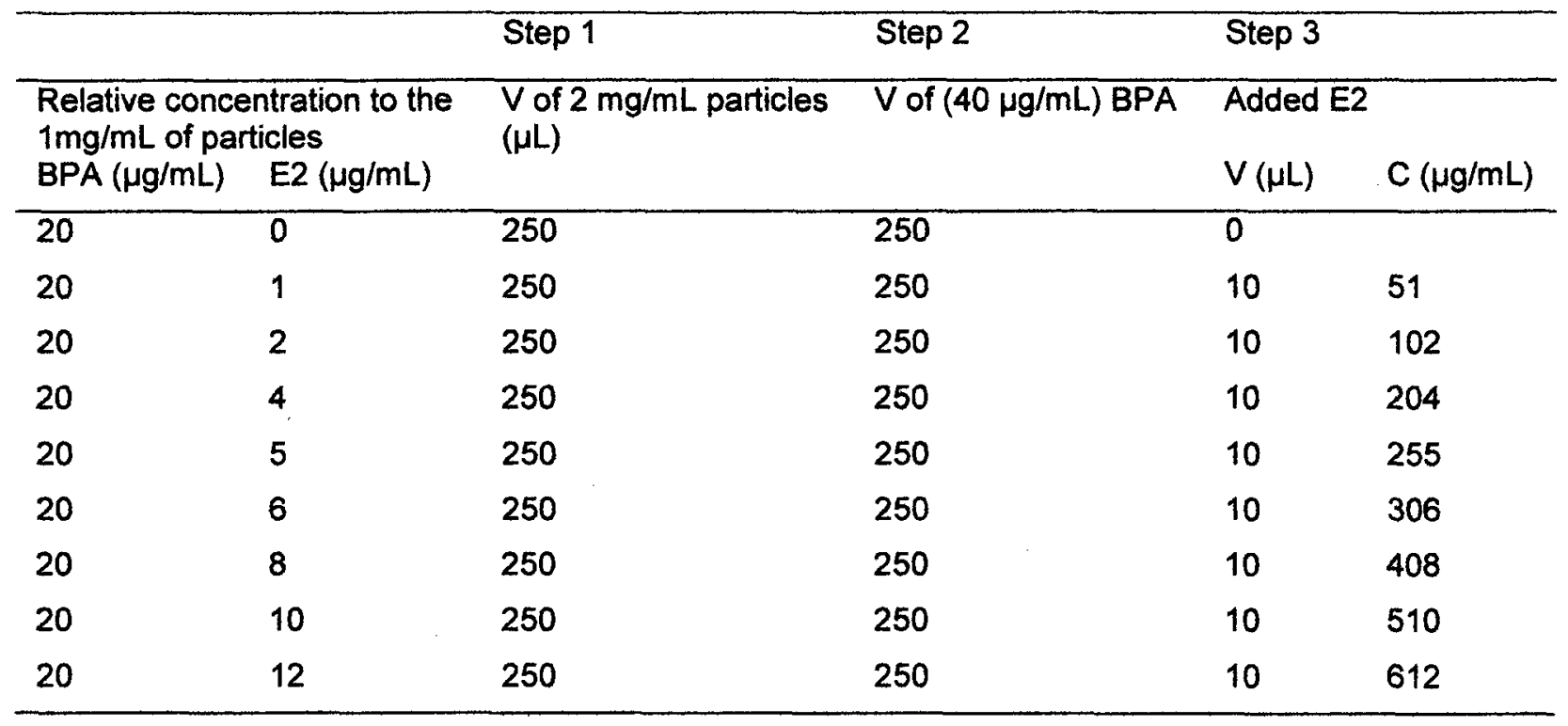




\section{CHAPTER III}

\section{RESULTS AND DISCUSSIONS}




\subsection{Synthesis and properties of MIP and NIP particles}

MIPs were traditionally synthesized by bulk polymerization methods in a porogenic solvent. A number of studies have shown that separation of target molecules achieved by bulk MIPs in SPE [72-75]. Even though bulk polymerization is truly simple, grinding and sieving are required to achieve the desired particle size. This is a labor intensive and wasteful process, and result in a broad size distribution, which limited their application in binding assays. Moreover, although porosity can be controlled by the monomer concentration, pore size distribution reduces the availability of analyte molecules to the binding sites. That is where polymerization of submicron particles is beneficial. To obtain spherical particles with diameters ranging from 0.1 to $1.0 \mu \mathrm{m}$, the precipitation technique has been applied for a variety of applications such as binding assay and capillary electrophoresis [76-79].

\subsubsection{Preparation of MIP and NIP submicron particles}

Different types of MIPs have been developed, using various functional monomers and crosslinkers with different mole ratios between, cross-linker and functional monomer to prepare either bulk polymer or spherical particles [77,80-82]. In addition, it was reported that, to decrease nonspecific binding on MIP particles, an excess amount of E2 molecules in polymerization would be helpful. Furthermore, fewer available cross-linkers would be used for preparation of particles, in contrast to bulk polymerization, which typically uses a large mole ratio of crosslinker to functional monomer [83].

In this research, three different MIP particles for E2 and corresponding NIP particles as control were synthesized. The use of MAA as the functional monomer was related to the fact that its carboxylic group can act as a proton donor, as well as a hydrogen bond acceptor. In 
addition, MAA was suitable for template such as E2 containing Bronsted-basic or hydrogen bonding, depending to the situation. The cross-linkers stabilized the template-functional monomer complex in the polymerization process, therefore, formulated 3-dimensional binding cavities, which were available for binding with the target after removal of template. Moreover, the contribution of the cross-linker to molecular imprinting is mostly related to its ability to provide enough binding site accessibility and rapid mass transfer. EGDMA and TRIM with respectively two and three vinyl group were provided adequate mechanical and thermal stability for the MIP particles. Additionally EGDMA and TRIM were preferred as cross-linker for synthesizing MIP particles, as they provided sufficient wettability in most binding media, which is essential for their application in binding assays. Initially, MIP particles were prepared based on an established procedure that employed EGDMA as the cross-linker (MIP-E) [70]. The original choice of 1:8:6.7 was demonstrated on the great binding capacity in bulk polymer by choosing MAA as functional monomer and EGDMA as cross linker in the mole ratio of 1:8:25 and also in other report 1:7:12.5 for spherical particles synthesized by multi-step swelling polymerization procedure [81,84]. In addition, a previous study showed to obtain high binding specificity for MIPs templated with E2, a mole ratio of functional monomer to cross-linker in the range of 1.0:1 up to 2.3:1 was optimum [77]. An access of functional monomer in comparison to the template was used to ensure the formation of the template-functional monomer complex. To synthesize all three types of MIP particles, volumes of porogen were chosen as $40 \mathrm{~mL}$, which were essential for preparation of monodispersed spheres (monomer content $<11 \mathrm{vol} \%$ in the prepolymerization solution) [85]. Therefore, for the preparation of these submicron particles, the polymerization process was started from a diluted homogeneous monomer solution. This dilution ensured that as much of the functional monomer in comparison with the template as 
possible were available. In the other side, it warranted as little of the cross-linker for each mole of functional monomer as possible were available. These two limited availabilities can enhance the selectivity of product to the template. Furthermore, the less amounts of cross-linker were in contrast to the possible bulk polymerization. These particles were used for further study on binding properties in aqueous solution, and to develop a procedure for the characterization of particles using CE. Due to their small size and spherical shape, sub-micron particles are potentially suitable for characterization study by CE. MIP particles cross-linked with TRIM were synthesized in two mole ratios to obtain MIP particles (MIP-T4 and MIP-T7) in smaller diameters and ideally better binding performance. Moreover, the effect of less availability of cross-linker to functional monomer on the selectivity was studied. The result in the following chapters has been proven that the cross-linker may provide part of the binding site functionality to the imprinting, which overall showed in the MIP cross-linked with TRIM in a mole ratio of 1:8:7 resulted in smaller size, better selectivity, larger specific binding affinity and imprinting factor. All three types (E, T4 and T7) of MIP particles, and the corresponding NIP particles, were dispersed uniformly as a suspension in aqueous solution. But as in general, smaller MIP particles $(<500 \mathrm{~nm})$ were obtained; it could keep particles in suspension better so MIP particles cross-linked with TRIM are preferable for binding assay.

Bleeding of template from MIP-T7 and MIP-T4 particles before removal of template in the presence of various concentrations of E2 were examined. The results in Figure 7, reflected that significant amounts of E2 were bled to total concentration of $0.8 \pm 0.2 \mu \mathrm{g} / \mathrm{mL}$. This could indicate the non-specific binding of the particles, which were in equilibrium with template bleeding. Besides the constant final concentration of E2, could be related to limited solubility of $\mathrm{E} 2$ in the aqueous solution. 


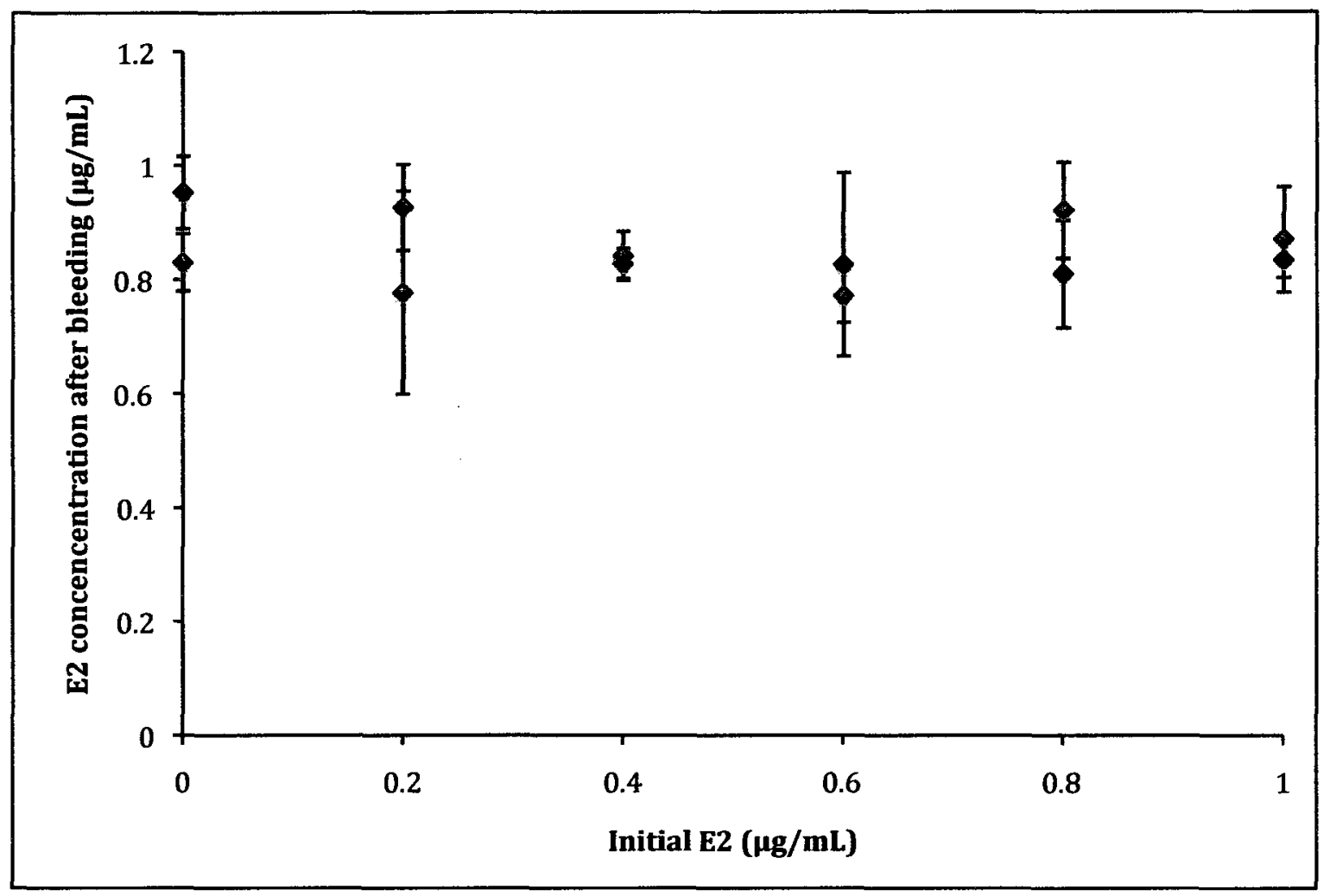

Figure 7 Bleeding template in MIP-T7 $(\diamond)$ and MIP-T4 $(\diamond)$ particles before removal of template.

The bled template in the solution could directly influence the detection limits of target analyte in case of using these particles as pre-concentration agent for trace analysis of E2. In addition, it could effect in the results of binding characterization of MIP particle by incomplete removal of E2 molecules. One solution to overcome these types of problem was suggested as the replacement of template with a structural analog to the analyte $[65,86]$. However, it is difficult to obtain such an analog for all templates, and although it could solve the influence of the detection of target analyte but before removal of template the available binding cavity would be so limited.

Therefore at the moment, the partial method to solve the problem is to increase the efficiency of removal of template to reduce the amount of bleeding to the lowest possible level. 
Several extraction methods, such as Soxhlet extraction (SE) [87], washing on-line and solid phase extraction were often reported [88].

In this work, in order to interrupt binding between E2 and MIP particles, TEA with $\mathrm{pk}_{\mathrm{a}}$ of 10.7 were selected as one of the strongest organic bases. Various concentrations of TEA (3$10 \%)$ in the methanol and mixture of methanol and DDW $(1: 10$ and 1:40 V/V) were applied for removal of template from MIPs. Due to limited solubility of E2 in aqueous solution up to $(\sim 15 \mu \mathrm{g} / \mathrm{mL})$ addition of DDW to the solution was not favorable. The $5 \%$ TEA in the methanol was resulted in quantitative removal of template after 6-8 times of washing with the solution. Supernatant of fifth wash were tested by HPLC-FD for quantification of possible remaining E2. After approval of no further bleeding of the template molecules, TEA were removed by acetonitrile leaving the cavity free, for further binding applications. In comparison with soxhlet extraction, this technique was quicker and easier to obtain.

\subsubsection{MIP and NIP particles size}

Molecularly imprinted polymer's size was assessed with scanning electron microscopy (SEM). SEM examination of MIP and NIP particles cross-linked with EGDMA (MIP-E, NIP-E) showed an average size of $0.6 \pm 0.1$ and $0.4 \pm 0.1 \mu \mathrm{m}$ respectively (Figure 8). Their dynamic diameter, as measured by a Nano-DLS particle size analyzer, was to be $578 \pm 23 \mathrm{~nm}$ (Figure 9). 


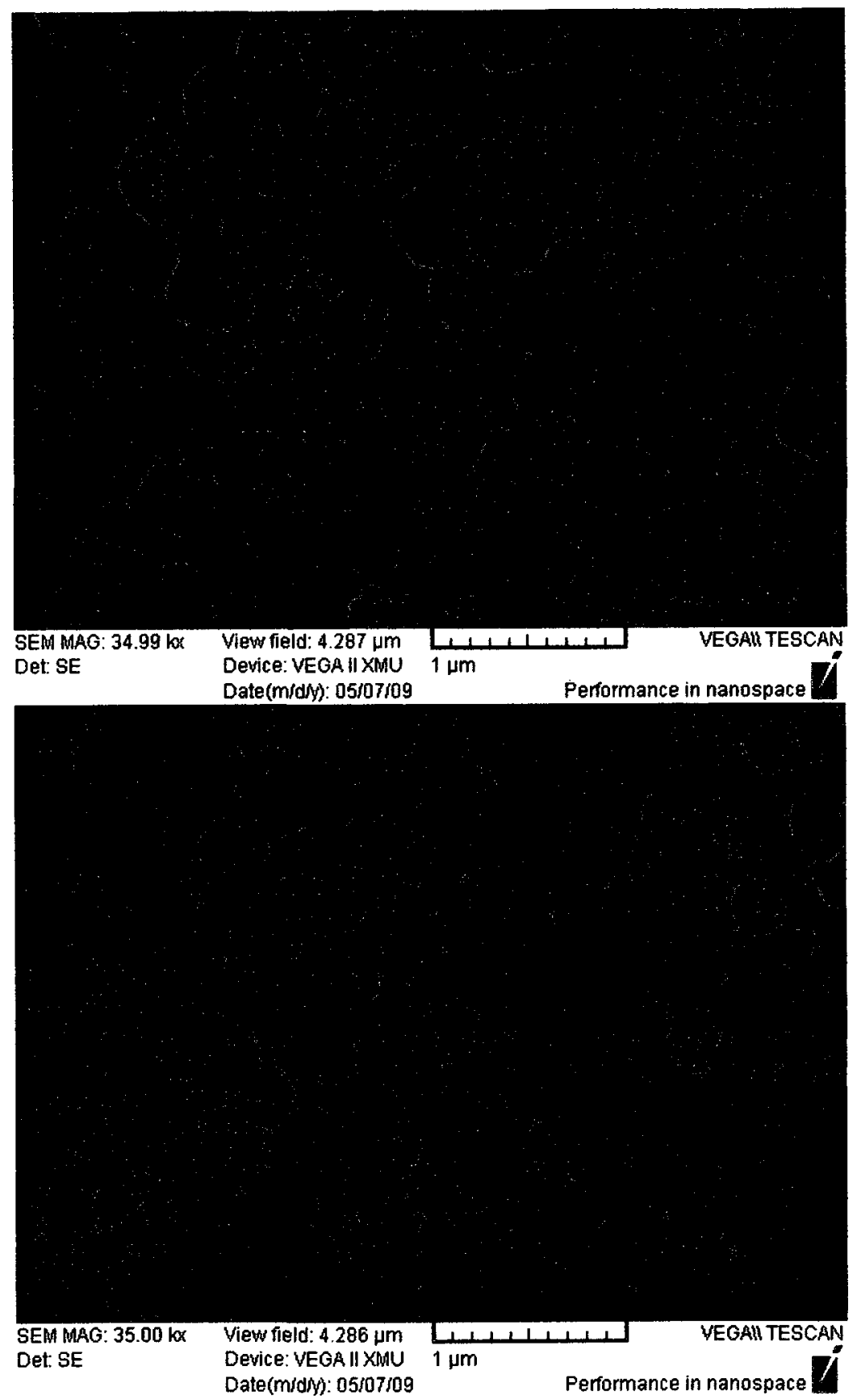

Figure 8 SEM image of a) MIP-E, b) NIP-E, magnification of $35 \mathrm{kX}$, acceleration voltage $20 \mathrm{kV}$. 


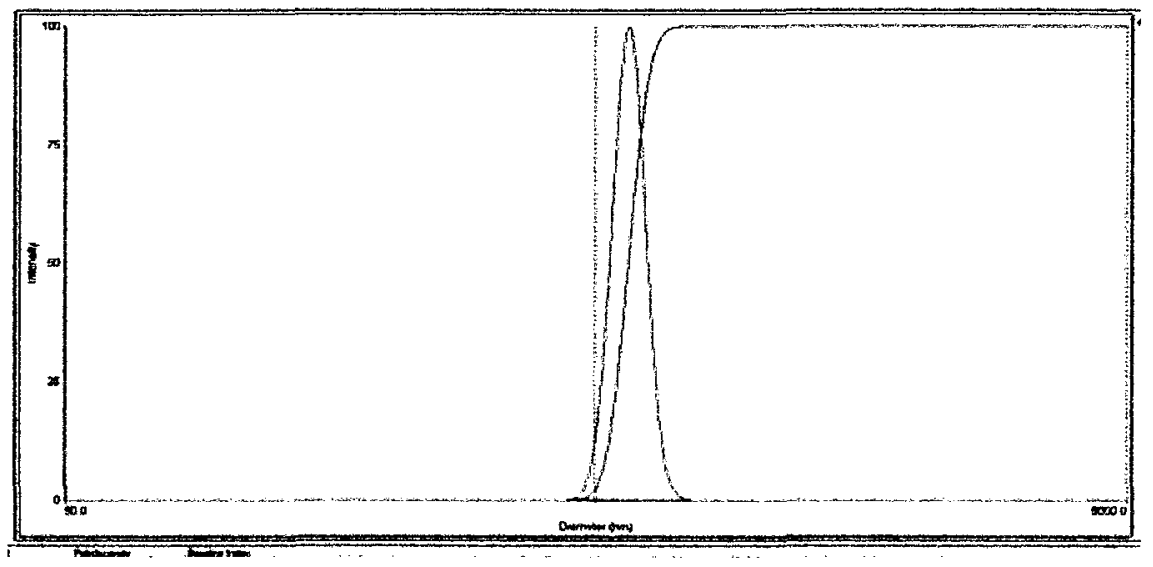

Figure 9 Typical effective size distribution of MIP-E particles obtained by nano- DLS particle size analyzer.

In using TRIM as a 3-dimensional cross-linker instead of EGDMA, two different mole ratios, were applied. Both the SEM images and DLS results showed a smaller diameter (size) for these MIP particles and corresponding NIP particles (Figure 10). This could be caused by tightly cross-linked 3-dimensional structure [67]. However, by applying a mole ratio of functional monomer to cross-linker as 8:7, smaller MIP particles were obtained resulting in a larger specific surface area. Hence, it was concluded that a 1:1 mole ratio for high binding specificity, which was previously reported by Ye et al. [77], is more favorable. 


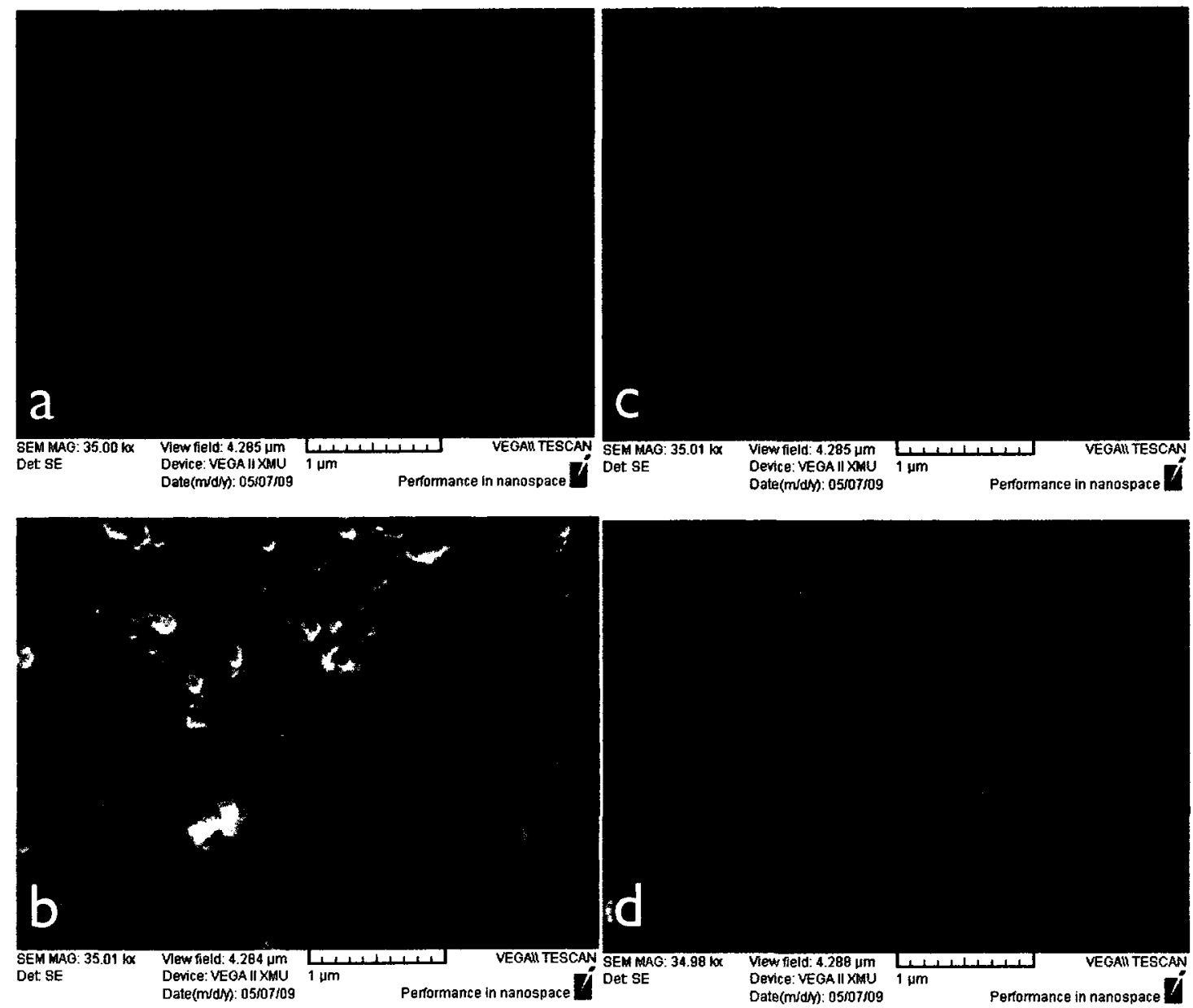

Figure 10 SEM images of (a) MIP-T7, (b) NIP-T7, (c) MIP-T4, and (d) NIP-T4. Magnification of $35 \mathrm{kXwas}$ afforded by acceleration voltage of $20 \mathrm{kV}$.

Diameters of all types of MIP and NIP particles, as characterized by Nano-DLS particle size analyzer, are reported in Table 4.

Table 4 Diameters of different synthesized polymer obtained with DLS and SEM.

\begin{tabular}{lll}
\hline Polymer & Dynamic diameter obtained by DLS $(\mathrm{nm})$ & Diameter obtained SEM $(\mu \mathrm{m})$ \\
\hline MIP-E & $578 \pm 23$ & $0.6 \pm 0.1$ \\
NIP-E & $366 \pm 8$ & $0.4 \pm 0.1$ \\
MIP-T4 & $333 \pm 2$ & $0.4 \pm 0.1$ \\
NIP-T4 & $352 \pm 4$ & $0.3 \pm 0.1$ \\
MIP-T7 & $282 \pm 4$ & $0.3 \pm 0.1$ \\
NIP-T7 & $288 \pm 6$ & $0.3 \pm 0.1$ \\
\hline
\end{tabular}




\subsubsection{Batch binding experiment}

After preparation of different types of MIP and NIP particles, the binding properties of these particles were evaluated during batch binding experiments for characterization of binding capacity, binding efficiency, association constant and imprinting effect. Characterizing the binding properties of MIP particles is an essential factor towards improving the synthesis and application of these particles. Batch binding studies are characterization systems provided first understanding into the binding properties of the MIP particles. Scatchard plot is the most common approach for estimating the binding parameters of MIP particles in comparison with its corresponding NIP particles, which explains a linear relationship of particles in two high affinities, and low affinity region for the particles binding parameters [89].

\subsubsection{Binding capacity}

Typically, there are two types of high affinity and low affinity binding sites in MIP particles, which their binding parameters were assessed by Scatchard analysis. Equilibrium binding experiments for measuring the binding capacities of the different MIP and NIP particles were carried out by varying the $\mathrm{E} 2$ concentration from 1 to $10 \mu \mathrm{g} / \mathrm{mL}$ in DDW containing a fixed amount of particles $(0.25 \mathrm{mg} / \mathrm{mL})$. The obtained data were plotted according to the Scatchard equation (Eq. 8) to determine the association constant $\left(\mathrm{K}_{\mathrm{a}}\right)$ and the number of accessible sites (B) [89].

$\mathrm{n} / \mathrm{C}_{\mathrm{f}}=(\mathrm{B}-\mathrm{n}) \mathrm{K}_{\mathrm{a}}$ Equation 8

Where $\mathrm{n}$ is bound $\mathrm{E} 2$ and $\mathrm{C}_{\mathrm{f}}$ is the free $\mathrm{E} 2$. 
A plot of the ratios of bound E2 to the free $\mathrm{E} 2\left(\mathrm{n} / \mathrm{C}_{\mathrm{f}}\right)$ versus (n) at a fixed amount of particles is shown in Figure 11.

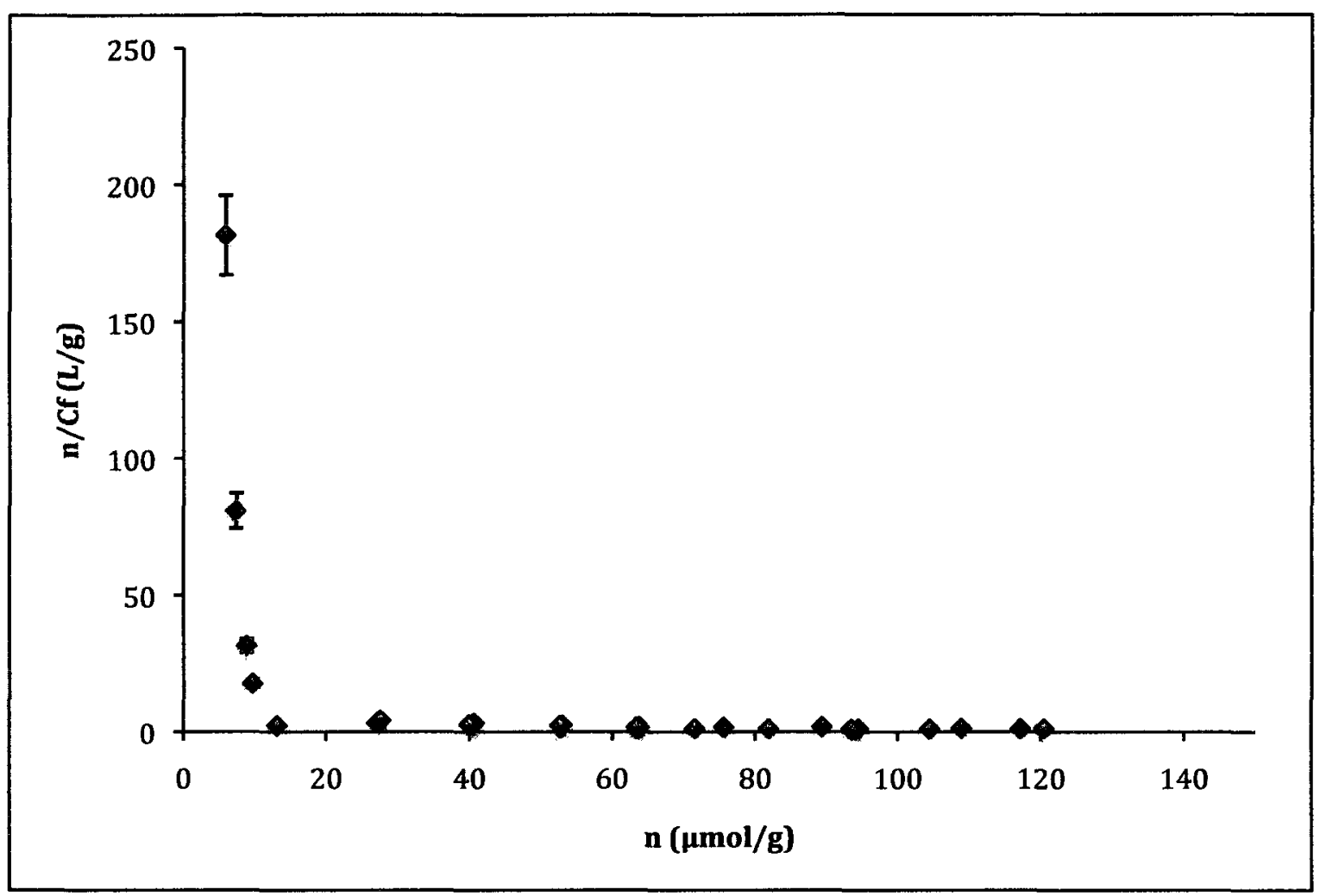

Figure 11 Scatchard plots for determination of binding properties of MIP-E $(\diamond)$ and NIP-E $(\diamond)$.

The plots are not linear, indicating that the binding sites in all types of MIP particles (MIP-T7, MIP-T4 and MIP-E) are heterogeneous concerning their affinity with E2. The two distinct sections in the plot indicate two classes of binding sites. The higher affinity binding sites (specific binding sites) of MIP-T7 particles can be characterized as $\mathrm{K}_{\mathrm{a}}=4.6 \times 10^{6} \mathrm{M}^{-1}$ and $\mathrm{B}=43 \pm 8 \mu \mathrm{mol} / \mathrm{g}$, from the slope and the intercept respectively (Figure 12). Similarly, the lower affinity binding sites (non-specific binding sites) have $\mathrm{K}_{\mathrm{a}}=2.3 \times 10^{5} \mathrm{M}^{-1}$ and $\mathrm{B}=105 \pm 10 \mu \mathrm{mol} / \mathrm{g}$, resulting in a maximum binding capacity $\left(\mathrm{B}_{\max }\right)$ of $150 \pm 15 \mu \mathrm{mol} / \mathrm{g}$ for MIP-T7. In comparison, the $K_{a}$ and $B$ values for the non-specific binding sites of NIP-T7 particles were assessed to be 
$1.2 \times 10^{5} \mathrm{M}^{-1}$ and $55 \pm 9 \mu \mathrm{mol} / \mathrm{g}$. The imprinting effect, I.E. $=\mathrm{B}_{\max }(\mathrm{MIP}) / \mathrm{B}_{\max }(\mathrm{NIP})$, can be calculated 3.0 \pm 0.8 for MIP-T7 [90].

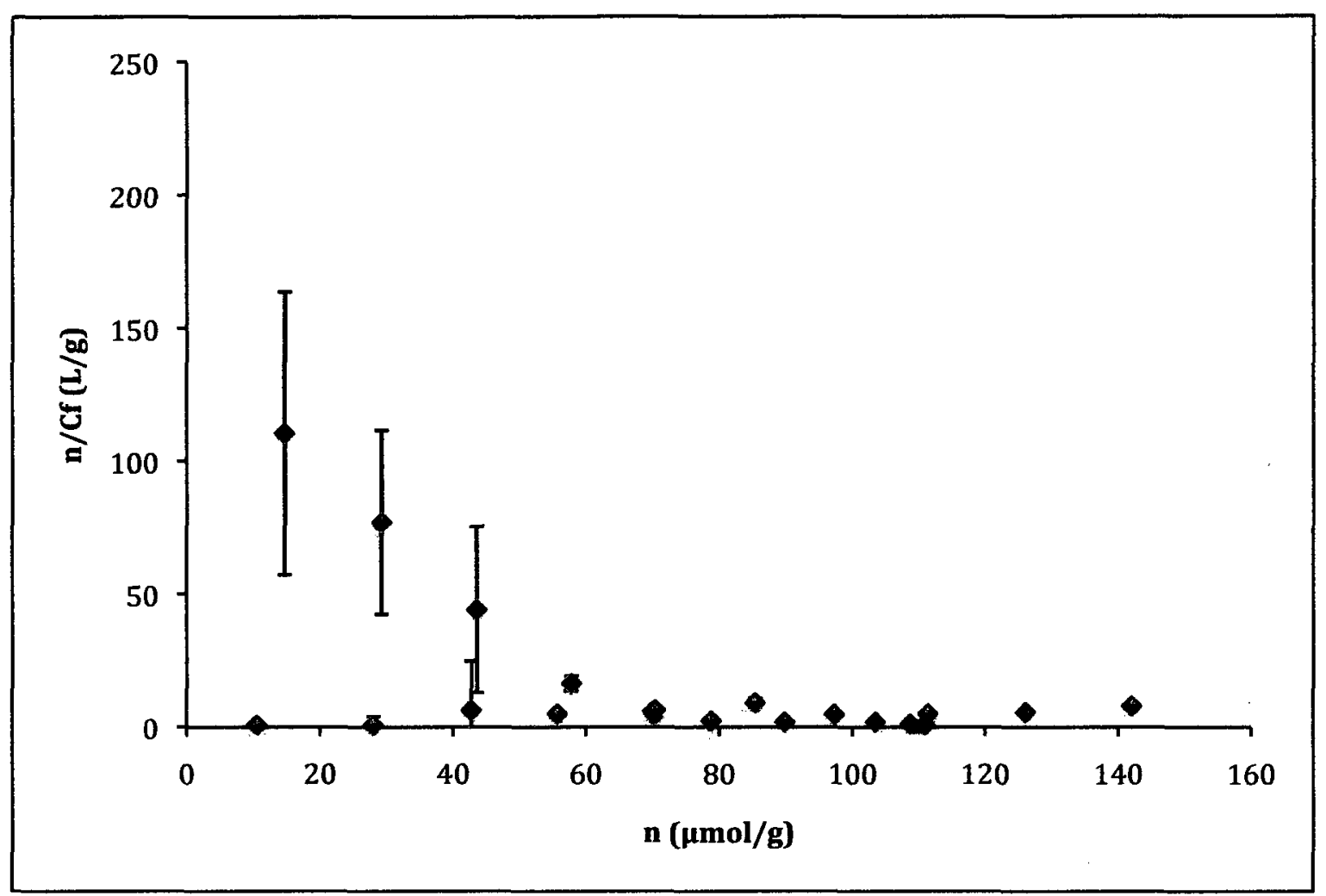

Figure 12 Scatchard plots for determination of binding properties of MIP-T7 $(\bullet)$ and NIP-T7 $(\diamond)$.

After scheming scatchard plot for MIP-T4 (Figure 13) the binding properties of MIP-T4 were calculated. The scatchard association constants and binding capacities of high-affinity and lowaffinity binding sites for all six types of MIP and NIP particles were summarized in Table 5. 


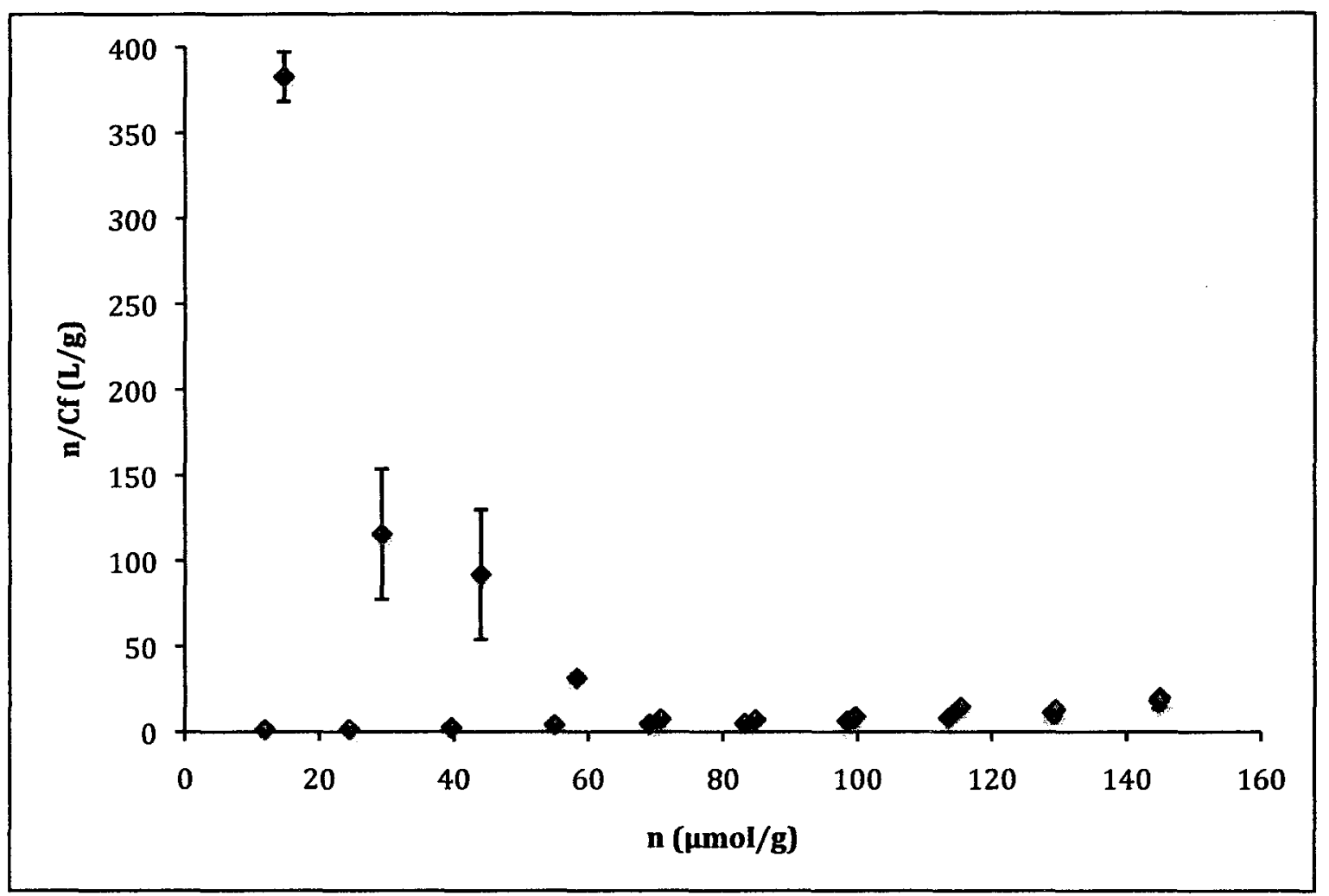

Figure 13 Scatchard plots for determination of binding properties of MIP-T4 $(\bullet)$ and NIP-T4 $(\bullet)$.

As it is summarized in Table 5, there are large binding capacities for MIP particles cross-linked with three-dimensional TRIM which were agreed with the previous study on Z-L-Tyr-OH by Kempe et al [91]. In addition, the large numbers of low affinity (nonspecific) binding sites could be explained by access quantity of functional monomer, which, were used to ensure the formation of template-functional complex. 
Table 5 Association constant and binding capacities of high-affinity and low-a affinity binding sites of particles by scatchard data analysis.

\begin{tabular}{llllll}
\hline Polymer & Specific & \multicolumn{5}{c}{ Non-specific } \\
& $\mathrm{K}_{\mathrm{a}}\left(\mathrm{M}^{-1}\right)$ & $\mathrm{B}\left(\mu \mathrm{molg}^{-1}\right)$ & $\mathrm{K}_{\mathrm{a}}\left(\mathrm{M}^{-1}\right)$ & $\mathrm{B}\left(\mu \mathrm{molg}^{-1}\right)$ & Imprinting effect \\
\hline MIP-E & $4.4 \times 10^{6}$ & $8 \pm 3$ & $6.8 \times 10^{4}$ & $57 \pm 9$ & $1.8 \pm 0.5$ \\
NIP-E & & - & $9.8 \times 10^{5}$ & $37 \pm 8$ & - \\
MIP-T4 & $1.1 \times 10^{6}$ & $33 \pm 7$ & $3.4 \times 10^{4}$ & $150 \pm 15$ & $1.2 \pm 0.2$ \\
NIP-T4 & & - & $2.3 \times 10^{4}$ & $150 \pm 15$ & - \\
MIP-T7 & $4.6 \times 10^{6}$ & $43 \pm 8$ & $2.3 \times 10^{5}$ & $105 \pm 10$ & $3.0 \pm 0.8$ \\
NIP-T7 & & - & $1.2 \times 10^{5}$ & $55 \pm 9$ & - \\
\hline
\end{tabular}

\subsubsection{Binding isotherm}

Binding isotherm of MIP-E particles were examined for determination of the minimum amount of particles which were required for binding of E2. The binding isotherm obtained from an initial E2 concentration of $1.0 \mu \mathrm{g} / \mathrm{mL}$ on different amounts of MIP particles. Results in Figure 14 fully illustrated the need for a sufficient amount of MIPs (5-10 mg) to completely remove $1 \mu \mathrm{g}$ of the estrogen under thermodynamic equilibrium. 


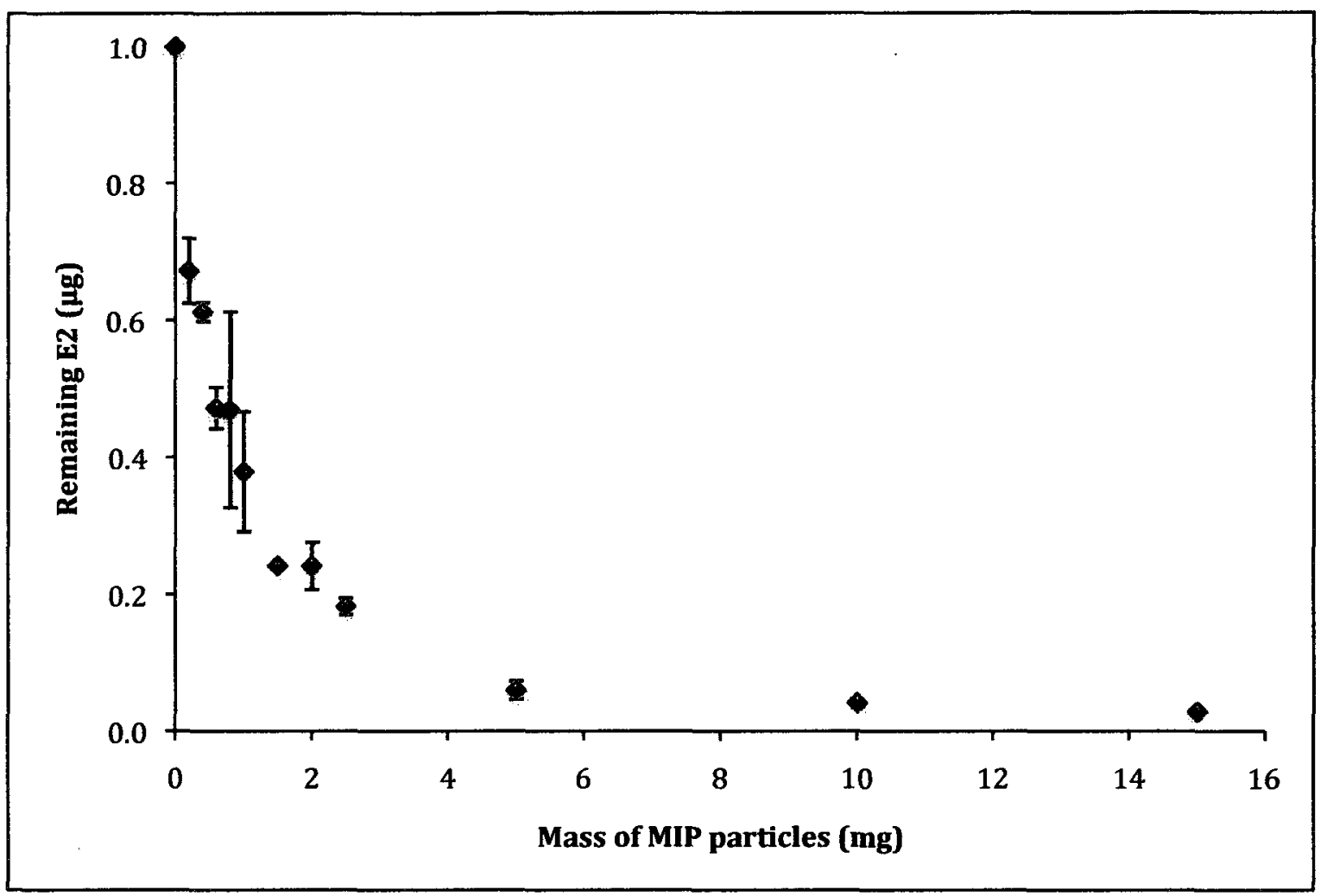

Figure 14 Determination of optimum amount of MIP-E for binding E2.

\subsubsection{Binding efficiency}

Binding efficiency of different types of MIPs and NIPs in comparison to each other were examined. As the first experiment binding efficiency of $20 \mathrm{mg}$ MIP-E particles after different incubation of 2, 15 and 60 minutes ensued a significant binding of $96.0 \pm 2.5 \%$ after short incubation of 2 minutes (Figure 15). 


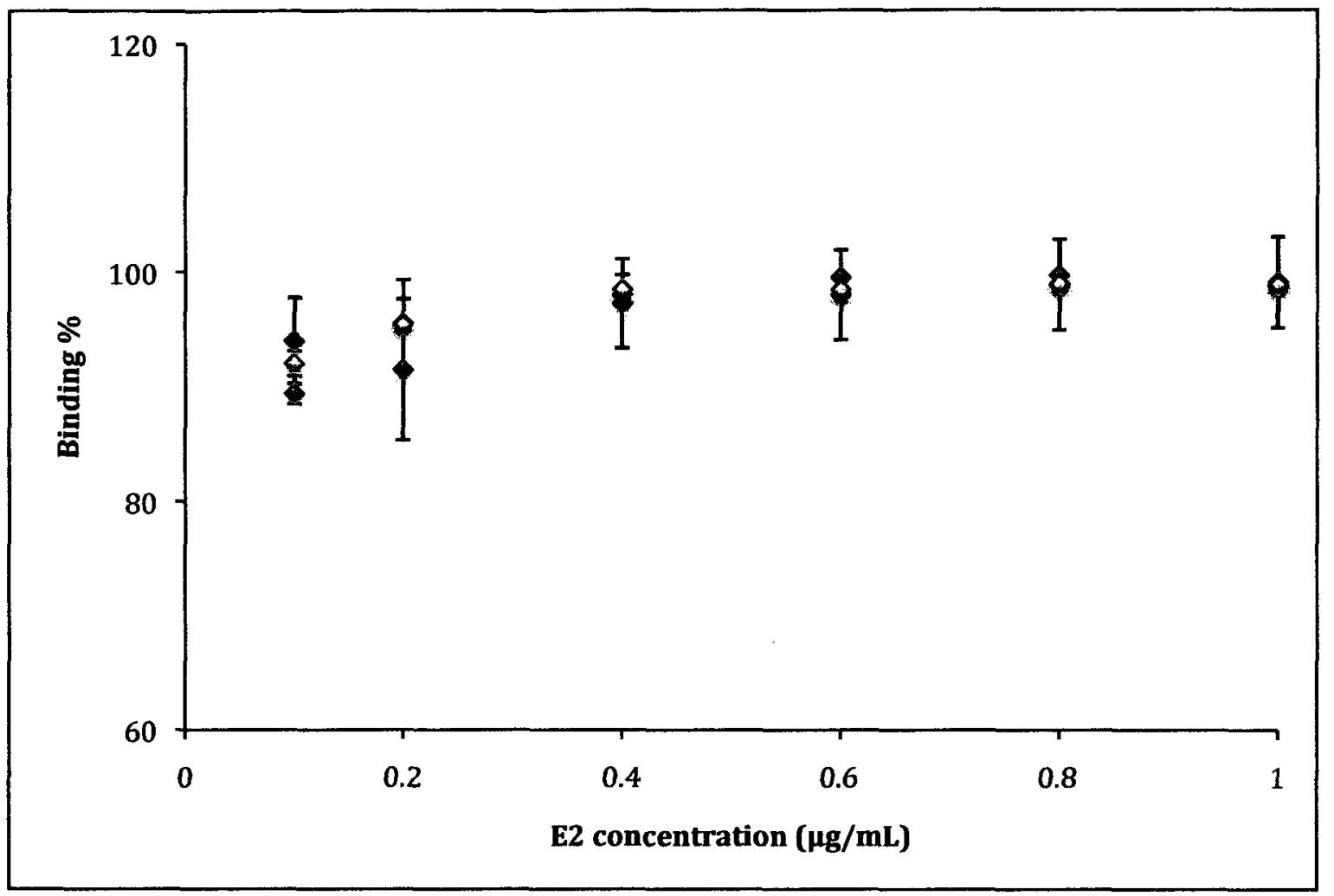

Figure 15 Binding efficiency of MIP-E after $2(\bullet), 15(\diamond)$ and $60(\diamond)$ minutes incubation.

Binding efficiencies of NIP-E in comparison to MIP-E after 2 minutes of incubations in Figure 16 indicated that by using NIP-E as expected, lower binding efficiency of $91.5 \pm 5 \%$ was observed. The difference in the E2 removal efficiency between MIP-E and NIP-E particles confirmed the small specific binding sites of MIPs comparing to corresponding NIP particles. 


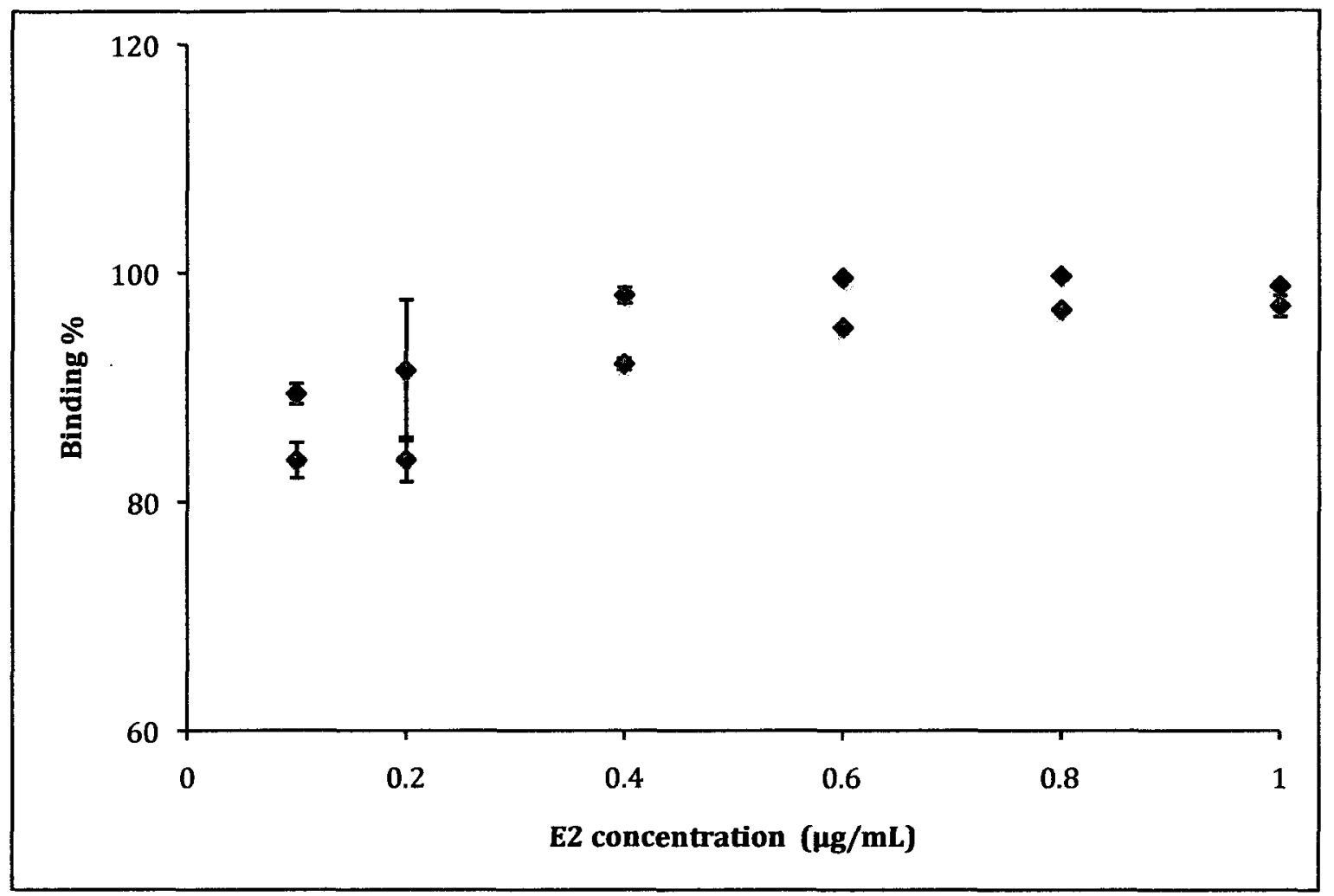

Figure 16 Binding efficiency of $20 \mathrm{mg}$ MIP-E $(\diamond)$ and NIP-E $(\bullet)$ after 2 minutes incubation.

As seen in Figure 17 compared to MIP-E and NIP-E, higher binding efficiencies of $99.0 \pm 0.5 \%$ and $93.0 \pm 1.5 \%$ for MIP-T7 and NIP-T7 were obtained. These particles are smaller in diameters, which resulted in larger surface area and higher binding efficiencies. 


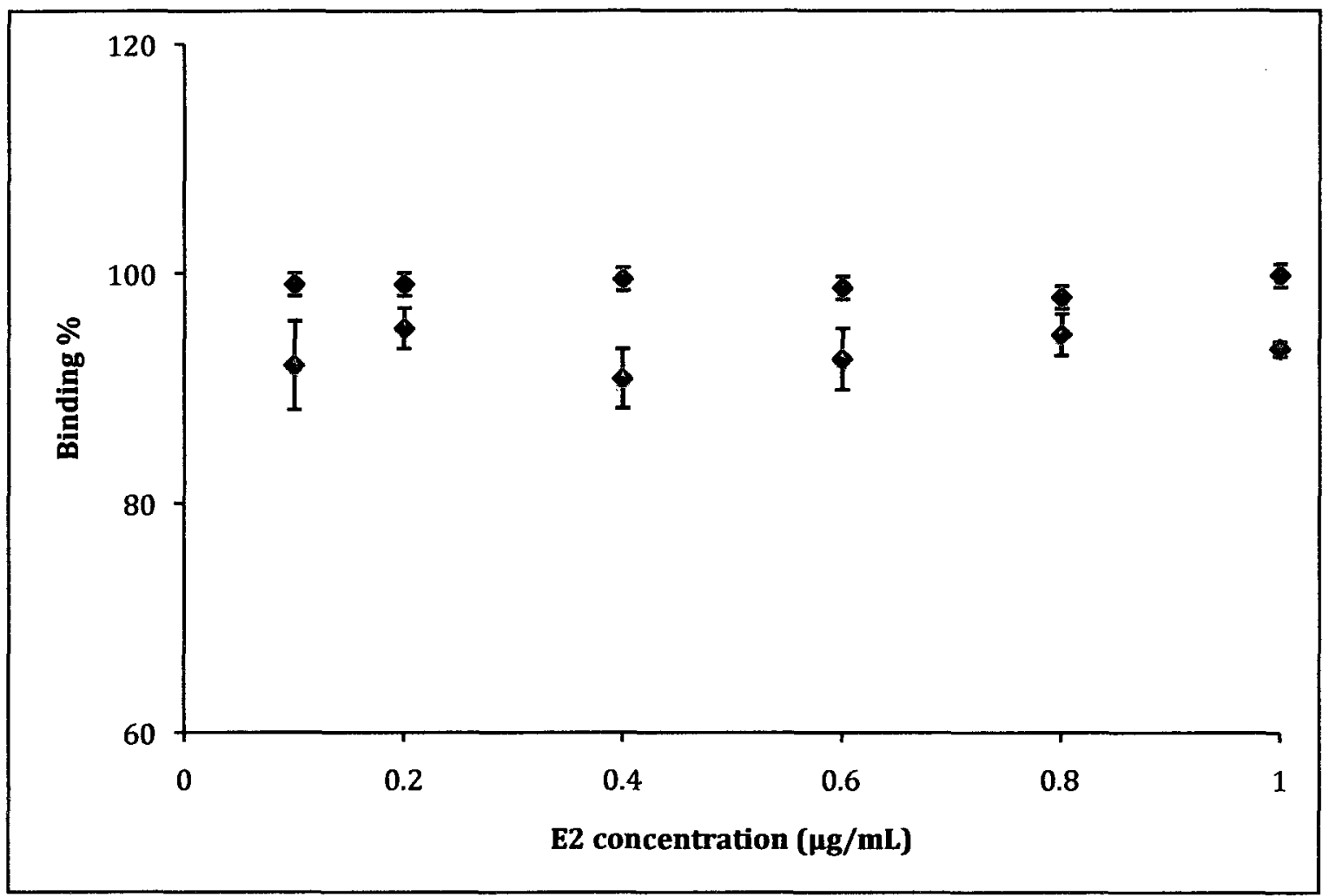

Figure 17 Binding efficiency of 5mg MIP-T7 $(\bullet)$ and NIP-T7 $(\bullet)$ after 2 minutes incubation.

Same experiment for particles cross-linked with smaller mole ratio of TRIM resulted averagely to the moderate binding efficiencies of $97.5 \pm 1.5 \%$ and $96.5 \pm 2.0$ for MIP-T4 and NIP-T4 particles respectively (Figure 18). The small difference in binding efficiencies between MIPs and NIPs were in agreement to the binding properties of these particles that suggested very small specific binding sites for MIPs and equal non-specific binding sites for both MIP and NIP particles. Besides, the result indicated that although there was a larger binding affinity for specific binding sites, but due to their limited availability and large availability of low affinity binding sites and also their relatively close affinity, there was not a significant difference in binding efficiencies of MIP-T4 and NIP-T4 particles. 


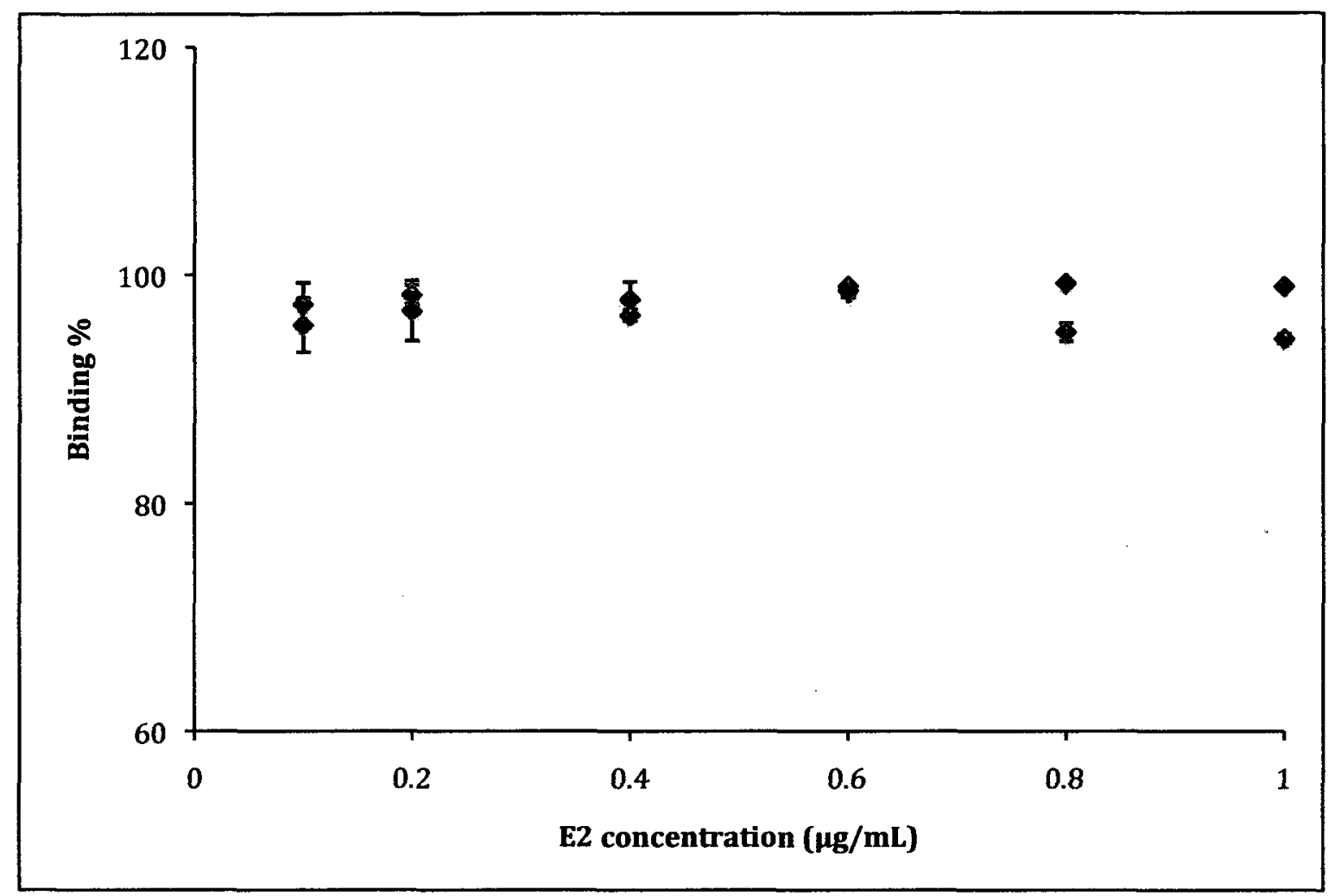

Figure 18 Binding efficiency of $5 \mathrm{mg}$ MIP-T4 $(\diamond)$ and NIP-T4 $(\diamond)$ after 2 minutes incubation.

The amounts of bound E2 on MIP-E particles in same unit of binding capacity $(\mu \mathrm{mol} / \mathrm{g})$ were summarized in Table 6 . The results clearly indicated when $20 \mathrm{mg}$ particles were used, the amounts of bound E2 on the particles were not anywhere close to the specific binding capacities of particles $(7 \mu \mathrm{mol} / \mathrm{g})$. As previously seen in Figure 15 binding efficiencies of particles were increased by increasing the initial E2 concentration.

In the other set of an experiment by using $0.5 \mathrm{mg}$ of MIP-E particles, binding efficiencies of particles were studied. As seen in the Figure 19, by increasing initial concentration of added E2 to the MIP-E particles, binding efficiencies of E2 decreased. Tables 6, revealed that by increasing the concentration of $\mathrm{E} 2$, the amount of $\mathrm{E} 2$ bound on to the particles were moved towards the specific binding capacities of the particles. Less availability of specific sites 
comparing to be widely available non-specific binding sites resulted in binding of target molecules on non-specific binding sites with the lower association constant.

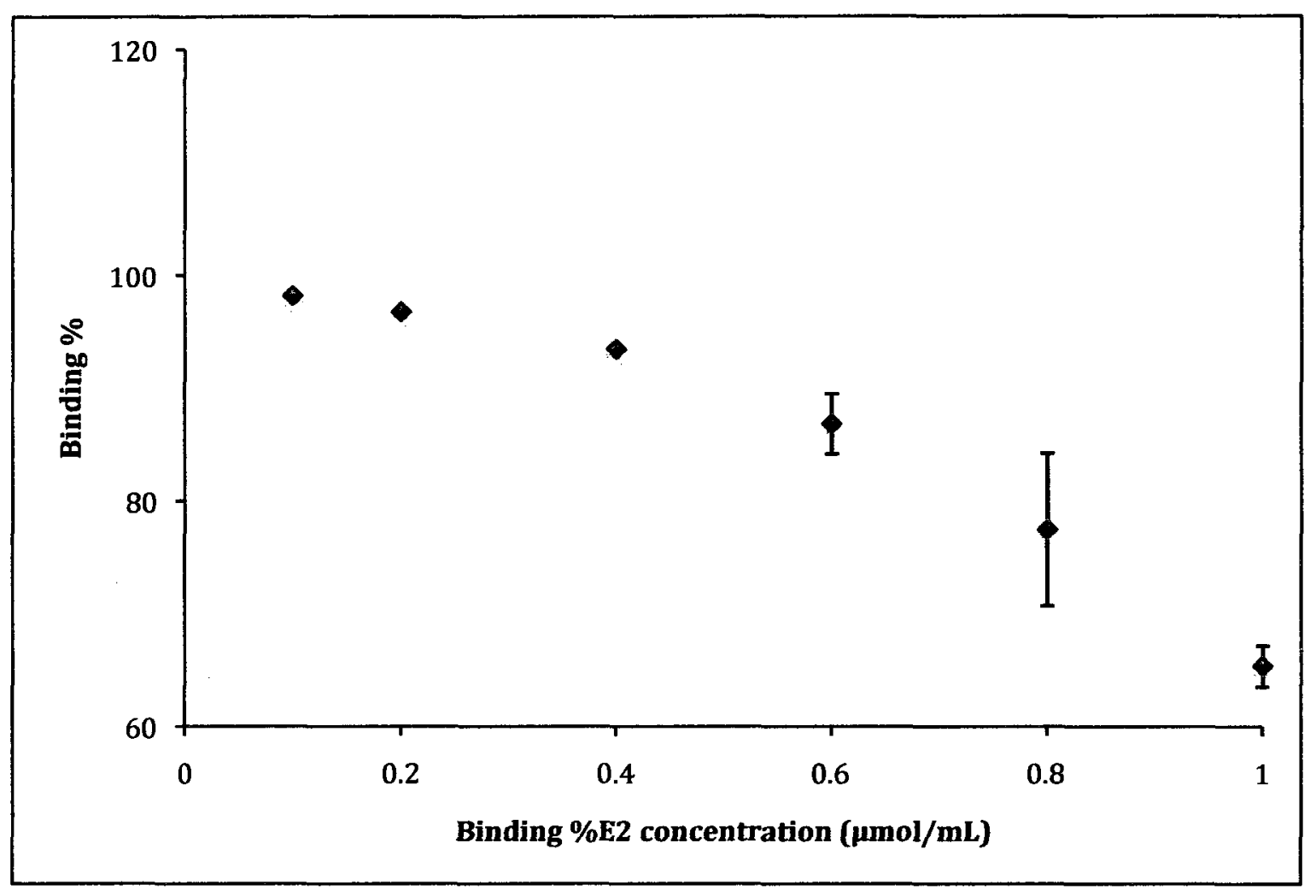

Figure 19 Binding efficiency of $0.5 \mathrm{mg}$ MIP-E after 2 minutes incubation.

In the other hand when $20 \mathrm{mg}$ of particles were used, increasing binding efficiencies of particles by increasing the concentration of E2 were described activation beyond the diffusion energy barrier for binding the target analytes. In the other word by increasing the concentration of E2 there would be more chance of E2 molecule to contact with specific binding sites inside MIP particles [92]. However, when $0.5 \mathrm{mg}$ of particles were used, by increasing the concentration of E2, although there is a better chance of E2 to contact with specific binding sites but due to 
limitation of them, E2 was bound on non-specific binding sites resulting in lower binding efficiencies.

Table 6 Amount of bound E2 per gram of MIP-E particles.

\begin{tabular}{|c|c|c|c|}
\hline \multicolumn{2}{|l|}{$20 \mathrm{mg}$ MIP-E } & \multicolumn{2}{|l|}{$0.5 \mathrm{mg}$ MIP-E } \\
\hline Initial E2 $(\mu \mathrm{g} / \mathrm{mL})$ & Bound E2 $(\mu \mathrm{mol} / \mathrm{g})$ & Initial E2 $(\mu \mathrm{g} / \mathrm{mL})$ & Bound E2 $(\mu \mathrm{mol} / \mathrm{g})$ \\
\hline$\overline{0.1}$ & 0.02 & 0.1 & 0.72 \\
\hline 0.2 & 0.03 & 0.2 & 1.42 \\
\hline 0.4 & 0.07 & 0.4 & 2.74 \\
\hline 0.6 & 0.11 & 0.6 & 3.83 \\
\hline 0.8 & 0.15 & 0.8 & 4.55 \\
\hline 1.0 & 0.18 & 1.0 & 4.80 \\
\hline
\end{tabular}

Amount of bound E2 on $0.5 \mathrm{mg}$ of MIP-T7 and MIP-T4 particles were presented in Table 7. Smaller amount of bound E2 in comparing to specific binding capacities of MIP-T7 $(43 \pm 8$ $\mu \mathrm{mol} / \mathrm{g})$ and MIP-T4 $(33 \pm 7 \mu \mathrm{mol} / \mathrm{g})$ showed that most of E2 molecules was bound on high affinity binding sites of MIPs resulting in high efficiencies in binding. Figure 20 and 21 indicated that by increasing the E2 concentration, unlike MIP-Es, no decreasing in binding efficiencies of MIP-T4 and MIP-T7 were observed. 
Table 7 Amount of bound E2 per gram of MIP-T4 and MIP-T7 particles.

\begin{tabular}{ll}
\hline Bound E2 on $0.5 \mathrm{mg}$ of MIP-T4 $(\mu \mathrm{mol} / \mathrm{g})$ & Bound E2 on $0.5 \mathrm{mg}$ of MIP-T7 $(\mu \mathrm{mol} / \mathrm{g})$ \\
\hline 0.72 & 0.72 \\
1.43 & 1.45 \\
2.88 & 2.87 \\
4.33 & 4.38 \\
5.76 & 5.77 \\
7.21 & 7.24 \\
\hline
\end{tabular}

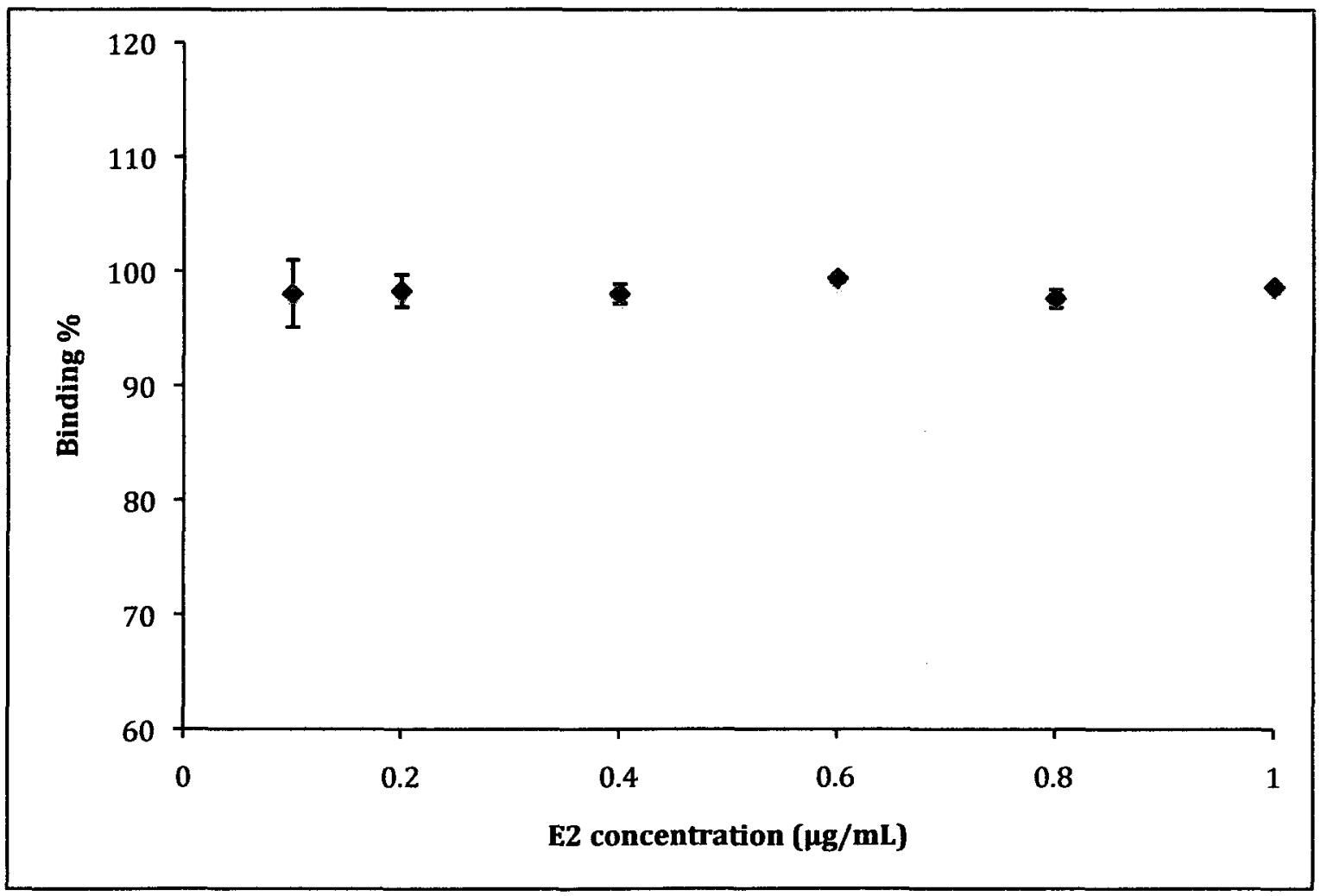

Figure 20 Binding efficiency of $0.5 \mathrm{mg}$ MIP-T7 after 2 minutes incubation. 


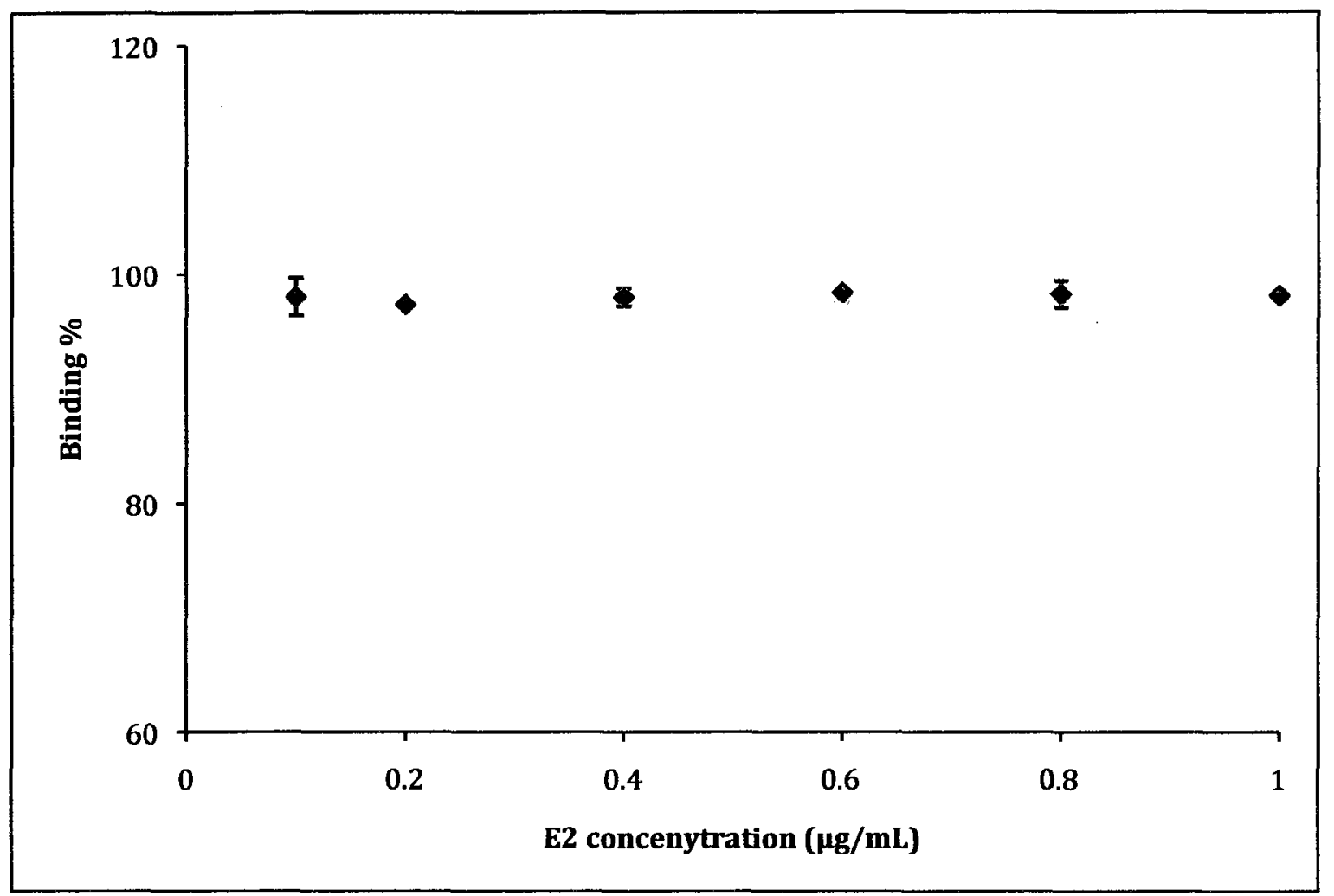

Figure 21 Binding efficiency of $0.5 \mathrm{mg}$ MIP-T4 after 2 minutes incubation.

After all, differences in binding efficiencies of various MIPs in comparison with corresponding NIPs (Figure 22-24) showed that MIP particles adsorbed more E2 than NIP particles. The differences in the E2 removal efficiency between MIP and NIP particles were agreed with imprinting effect of different types of synthesized polymer listed in Table 3. 


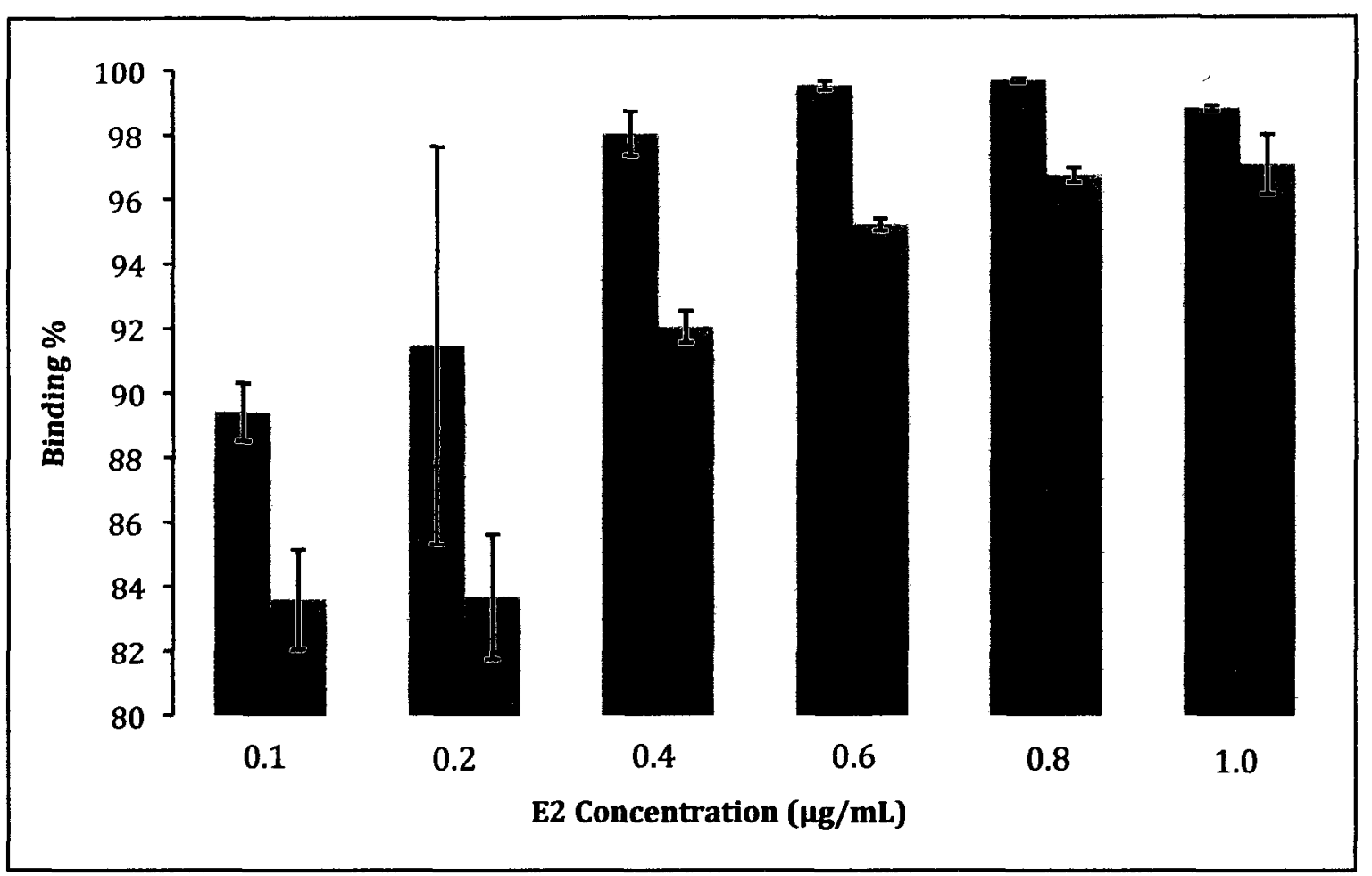

Figure 22 Binding difference of MIP-E (1) and NIP-E (I).

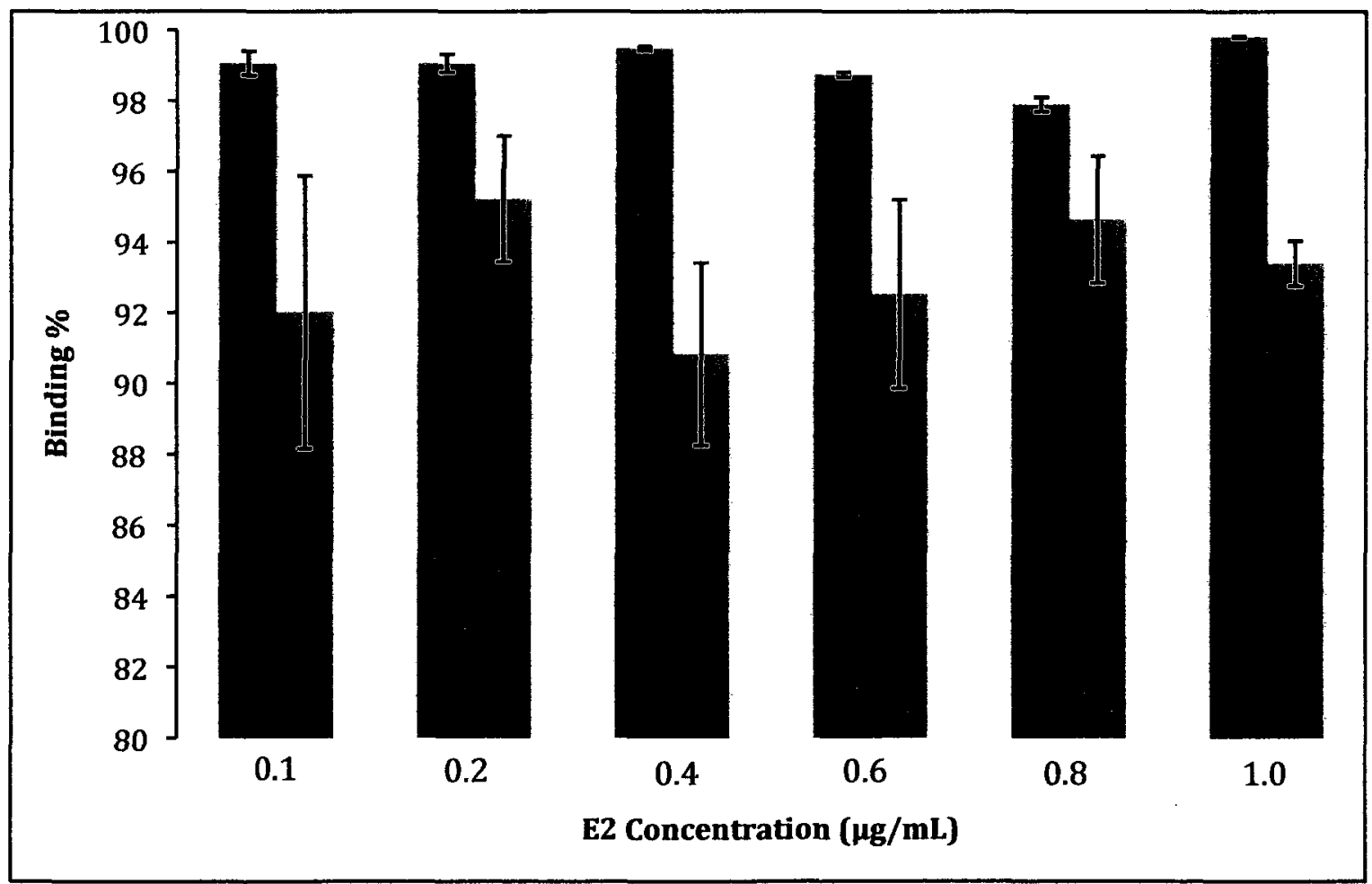

Figure 23 Binding difference of MIP-T7 (U) and NIP-T7 (U). 


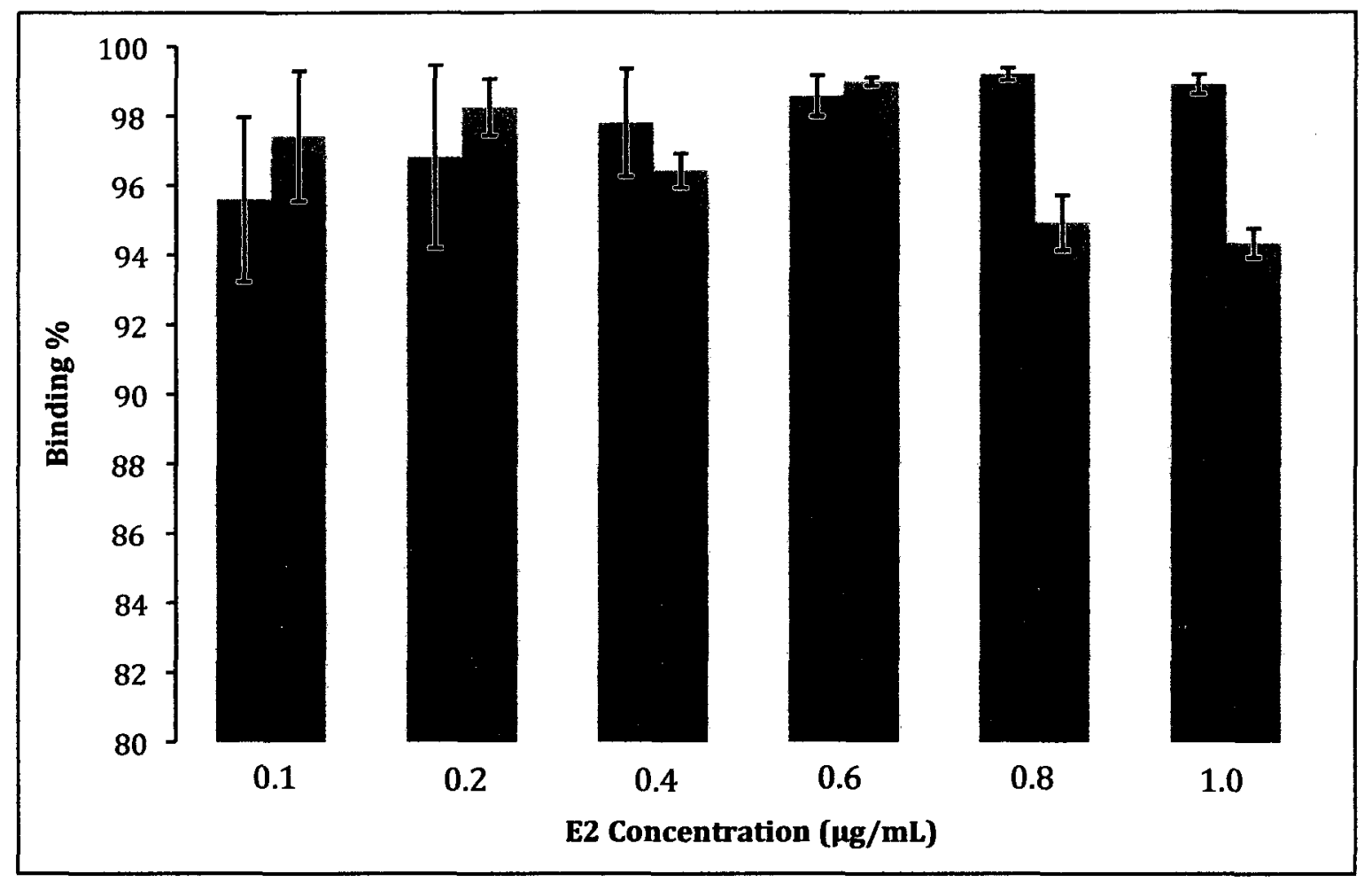

Figure 24 Binding difference of MIP-T4 ( 1 ) and NIP-T4 ( 1 ).

\subsubsection{Leakage of bound E2}

The Results in Table 8 indicated that after 5-minute incubations of various concentrations of E2 $(0.2-10 \mu \mathrm{g} / \mathrm{mL})$, averagely $97 \pm 1 \%$ of initial E2 were bound on to the different types of MIP-T4 and MIP-T7 particles. The result on leakage of bound E2 in DDW demonstrated that after 2 and 6 hours of incubations no leakage of bound E2 was detected (Figure 25). 


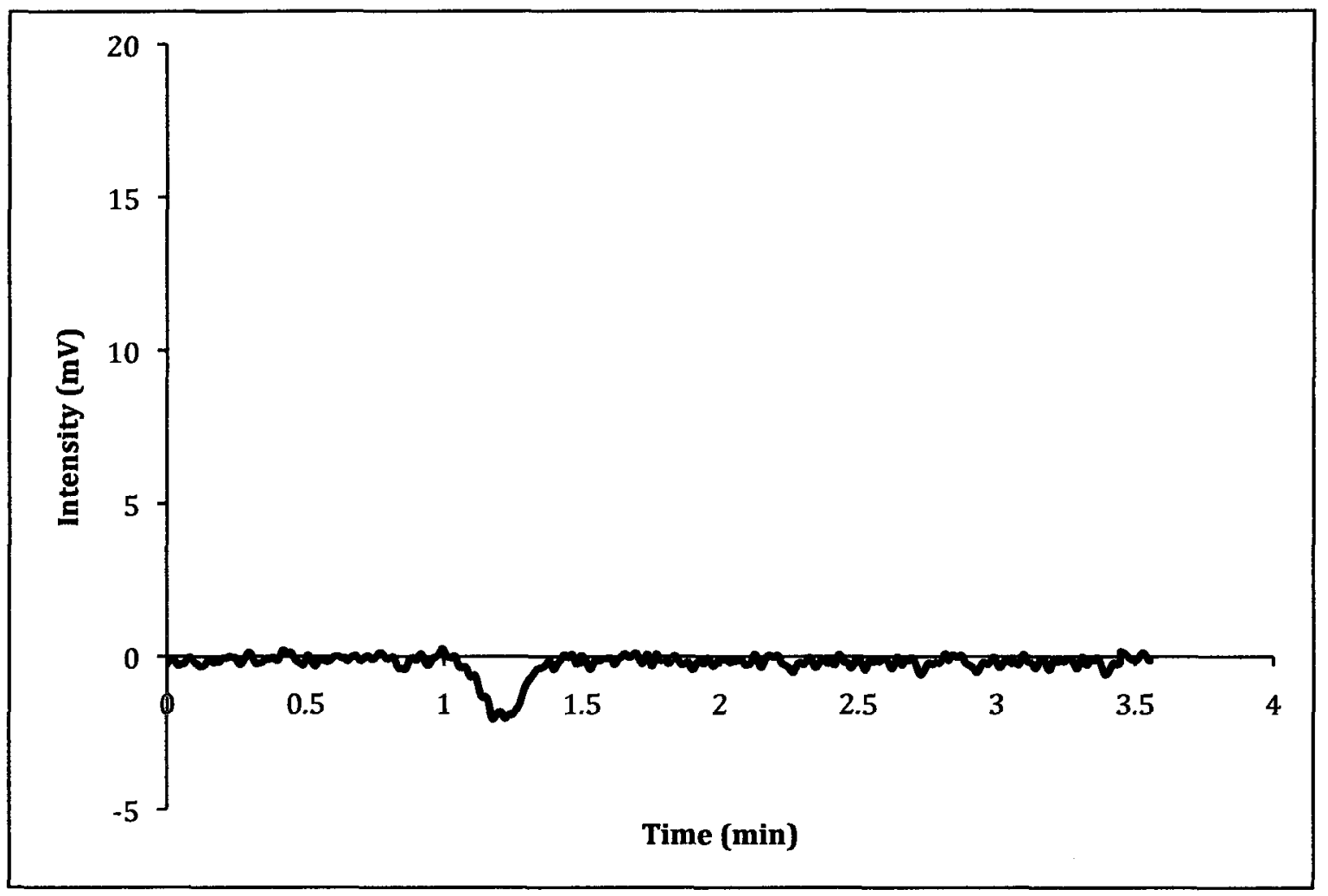

Figure 25 HPLC chromatograms of supernatant after 6 hours incubation of MIP-T4 particles in pure water.

One important aspect in this experiment was the detection limits of E2 which, determined to be $0.05 \mu \mathrm{g} / \mathrm{mL}$ (Figure 26) when Methanol: Acetonitrile: DDW in (1:1:2 v/v) were used as mobile phase. Observing no leakage could be due to strongly hydrophobic properties of the bound E2. Although such a characteristic, for example no leakage of E2 molecule after binding on MIPs was basic, but it is principal precondition for the application of MIP particles as an adsorbent in water and wastewater's pre-concentrations and treatment. 


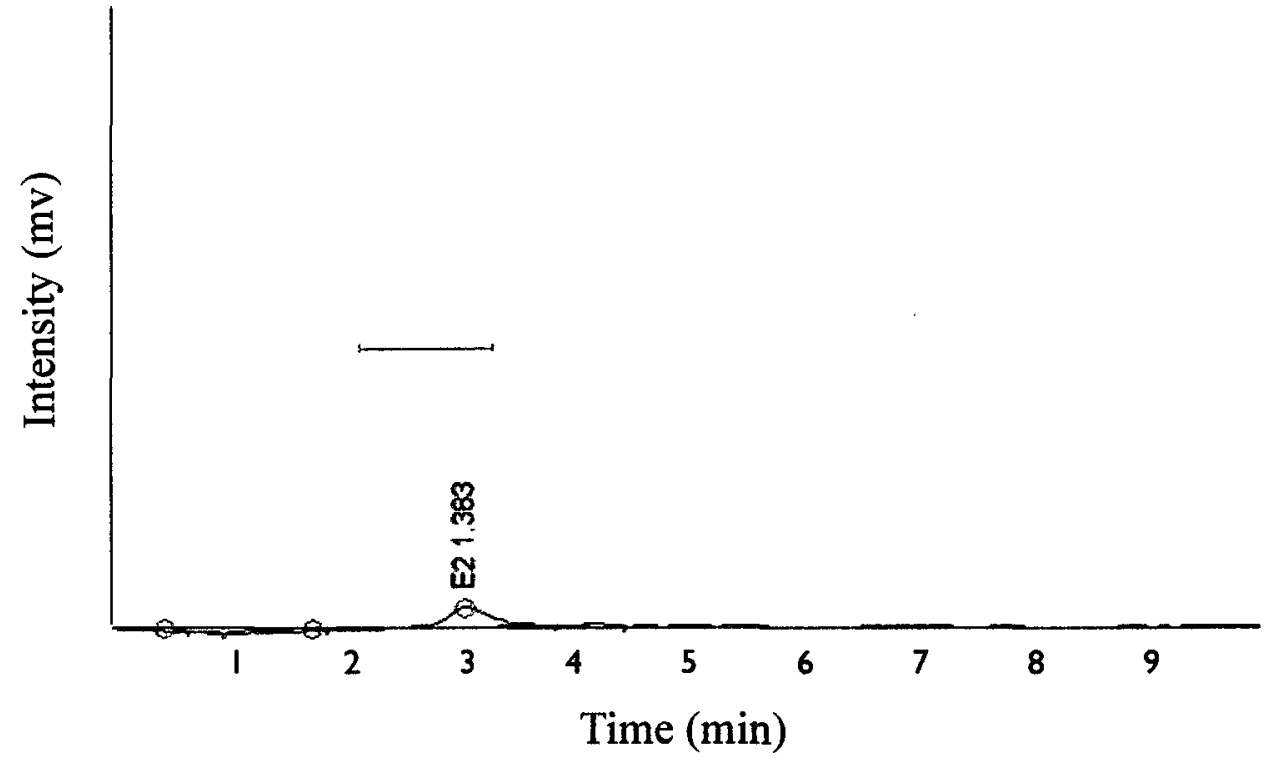

Figure 26 HPLC chromatograms $0.05 \mu \mathrm{g} / \mathrm{mL}$ of E2 in Aqueous solution.

Table 8 Amount of bound and leaked E2 on MIP-T4 and MIP-T7 particles.

\begin{tabular}{lllll}
\hline $\begin{array}{l}\text { Initial E2 } \\
(\mu \mathrm{g} / \mathrm{mL})\end{array}$ & $\begin{array}{l}\text { Bound E2 on MIP-T4 } \\
(\mu \mathrm{g})\end{array}$ & $\begin{array}{l}\text { Leaked E2 } \\
(\mu \mathrm{g} / \mathrm{mL})\end{array}$ & $\begin{array}{l}\text { Bound E2 on MIP-T7 } \\
(\mu \mathrm{g})\end{array}$ & $\begin{array}{l}\text { Leaked E2 } \\
(\mu \mathrm{g} / \mathrm{mL})\end{array}$ \\
\hline 0.2 & 0.20 & $\mathrm{n} / \mathrm{a}$ & 0.19 & $\mathrm{n} / \mathrm{a}$ \\
0.5 & 0.49 & $\mathrm{n} / \mathrm{a}$ & 0.48 & $\mathrm{n} / \mathrm{a}$ \\
1.0 & 0.99 & $\mathrm{n} / \mathrm{a}$ & 0.98 & $\mathrm{n} / \mathrm{a}$ \\
2.5 & 2.43 & $\mathrm{n} / \mathrm{a}$ & 2.43 & $\mathrm{n} / \mathrm{a}$ \\
5.0 & 4.77 & $\mathrm{n} / \mathrm{a}$ & 4.82 & $\mathrm{n} / \mathrm{a}$ \\
7.5 & 7.23 & $\mathrm{n} / \mathrm{a}$ & 7.28 & $\mathrm{n} / \mathrm{a}$ \\
10.0 & 9.37 & $\mathrm{n} / \mathrm{a}$ & 9.65 & $\mathrm{n} / \mathrm{a}$ \\
\hline
\end{tabular}




\subsection{Capillary Electrophoresis for characterization of MIP and NIP particles}

\subsubsection{Separation of MIP and NIP particles using CE}

CE electropherograms are shown in Figure 27 for individual injections of different types of MIP and NIP particles. Each sample was a suspension, containing $1 \mathrm{mg}$ of particles in $1 \mathrm{~mL}$ of 50 $\mathrm{mM}$ borate buffer $(\mathrm{pH}=8.5)$. It was introduced via hydrodynamic injection at $0.5 \mathrm{psi}$ for $1 \mathrm{~s}$. All types of MIP and NIP particles migrated after the neutral marker (mesityl oxide), yielding negative electrophoretic mobility values. Hence, the particles are negatively charged in the experimental conditions. As seen in Table 9, the electrophoretic mobility values of MIP and NIP particles containing TRIM are slightly different. This difference would result to application of CE on the separation of MIP and NIP particles which by best of our knowledge is obtained for the first time.

Table 9 Electrophoretic mobilities of MIP and NIP particles before and after addition of dynamic coating modifier.

\begin{tabular}{lll}
\hline Particles & $\mu_{\mathrm{ep}}\left(\mathrm{m}^{2} \cdot \mathrm{V}^{-1} \cdot \mathrm{S}^{-1}\right)$ & \\
& Before addition of EOTrol ${ }^{\mathrm{TM}} \mathrm{LN}$ & After addition of EOTrol ${ }^{\mathrm{TM}} \mathrm{LN}$ \\
\hline MIP-E & $-2.34 \times 10^{-8}$ & $3.21 \times 10^{-8}$ \\
NIP-E & $-2.34 \times 10^{-8}$ & $3.21 \times 10^{-8}$ \\
MIP-T4 & $-3.47 \times 10^{-8}$ & $3.75 \times 10^{-8}$ \\
NIP-T4 & $-3.84 \times 10^{-8}$ & $3.20 \times 10^{-8}$ \\
MIP-T7 & $-4.25 \times 10^{-8}$ & $4.15 \times 10^{-8}$ \\
NIP-T7 & $-4.81 \times 10^{-8}$ & $3.68 \times 10^{-8}$ \\
\hline
\end{tabular}




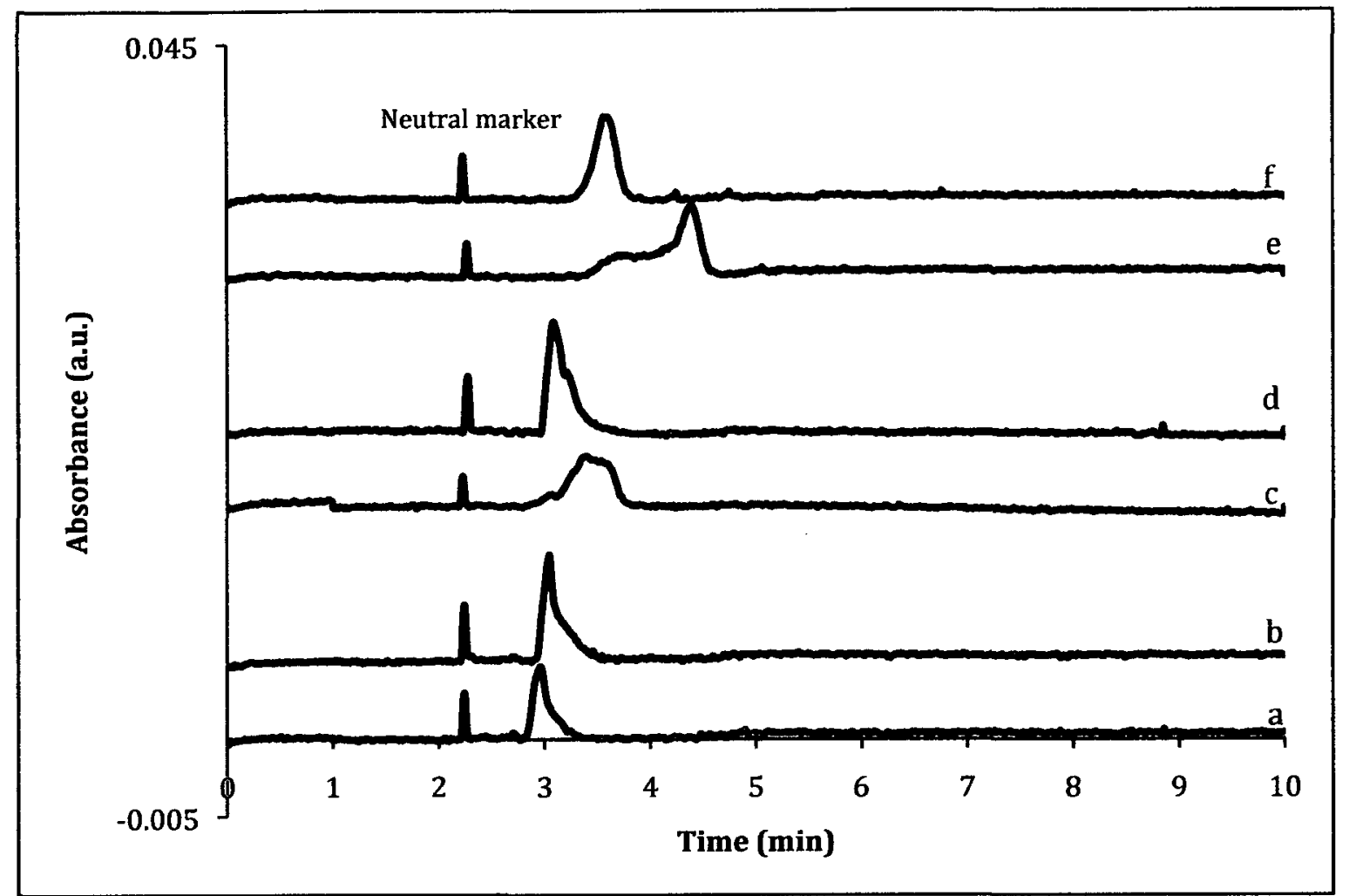

Figure 27 CE electropherograms of (a) MIP-E, (b) NIP-E, (c) MIP-T4, (d) NIP-T4, (e) MIP-T7, (f) NIP-T7 particles, in $50 \mathrm{mM}$ borate buffer $(\mathrm{pH}=8.5)$, separation voltage $30 \mathrm{kV}$; detection, $\mathrm{UV}$ at $214 \mathrm{~nm}$.

Different mixtures of MIP and corresponding NIP particles were prepared in the BGE. Each mixture was introduced via $1 \mathrm{~s}$ of hydrodynamic injection at $0.5 \mathrm{psi}$. Figure 28 and 29 indicated that separation of MIP-T4 and MIP-T7 from the corresponding NIP particles were successful. Discussions in Section 3.2.2.1 will explain that separation of MIP-T4 and NIP-T4 is based on the difference between their sizes. However, MIP-T7 and NIP-T7 were separated based on their charges. The smaller diameter of MIP-T4 in comparison with NIP-T4 resulted in a higher charge-to-size ratio. This pulled the MIP-T4 particles back towards the anode (at the capillary inlet) more quickly, resulting in a more negative electrophoretic mobility and hence later migration through the UV detection point (and out of the capillary). To characterize the MIP 
from corresponding NIP particles in Figures 28-29, the mobility value in Table-9 and individual CE electropherograms in Figure 27 were used as control.

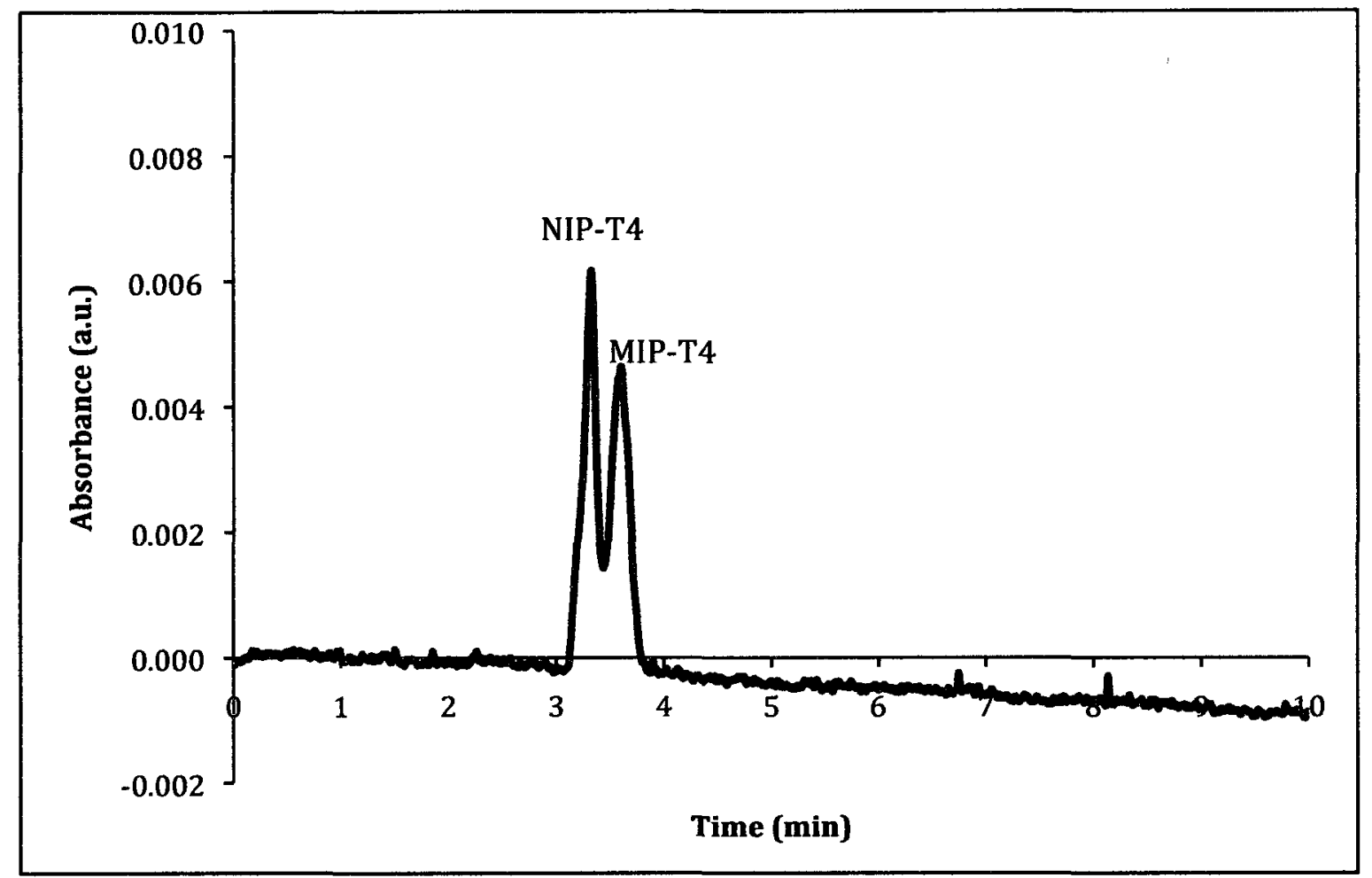

Figure 28 Separation of MIP-T4 and NIP-T4 by CE in $50 \mathrm{mM}$ borate buffer $(\mathrm{pH}=8.5)$. 


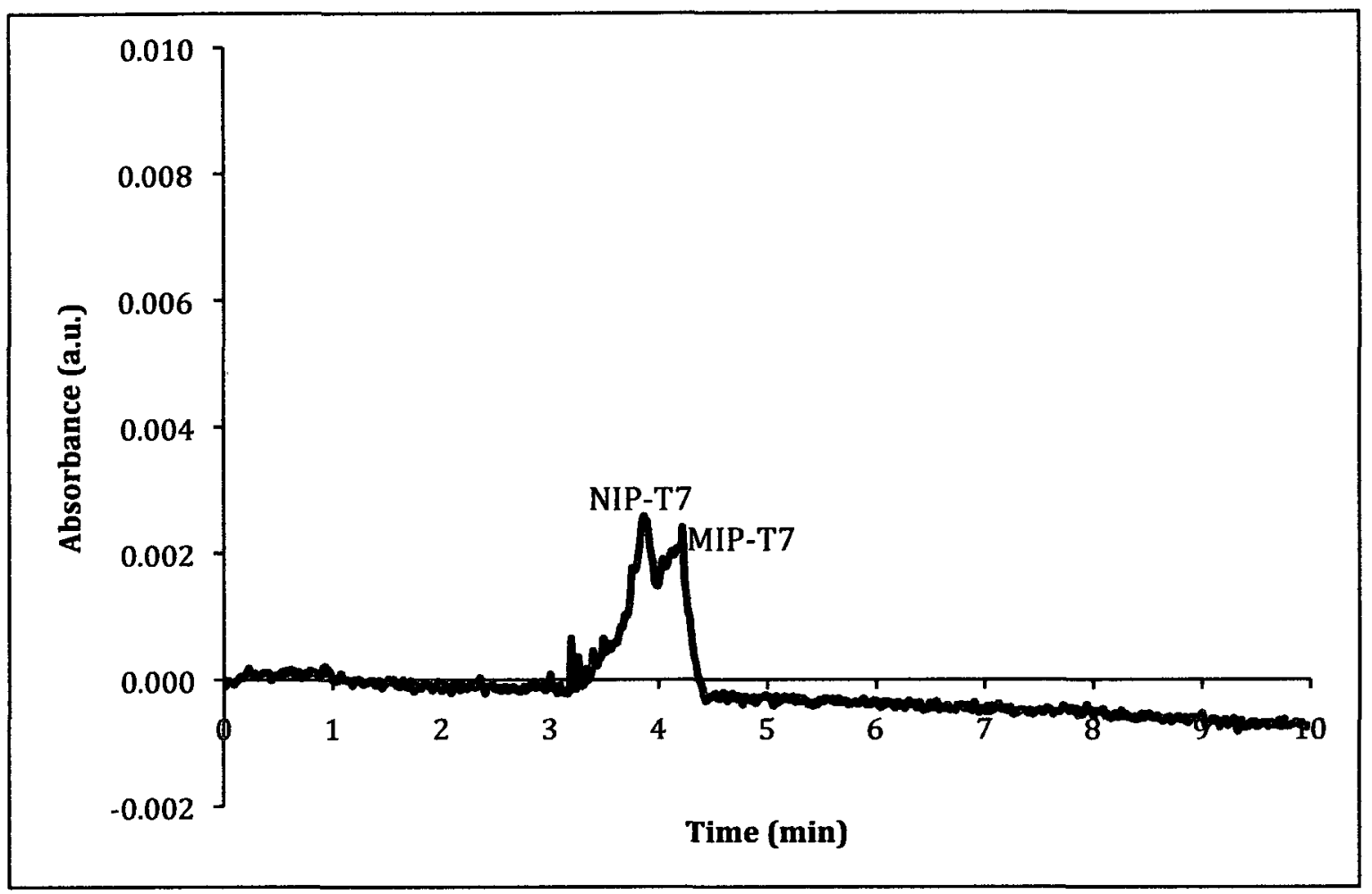

Figure 29 Separation of MIP-T7 and NIP-T7 by CE in $50 \mathrm{mM}$ borate buffer $(\mathrm{pH}=8.5)$.

As seen in Table 9 there is no difference in electrophoretic mobility of MIP-E and NIP-E. Although these particles are significantly different in their diameter but there is not any separation occurred (Figure 30). As it is discussed in Section 3.2.2.1 this could be used as a tool for prediction of non-specific binding characterization of MIP compared to NIP particles. 


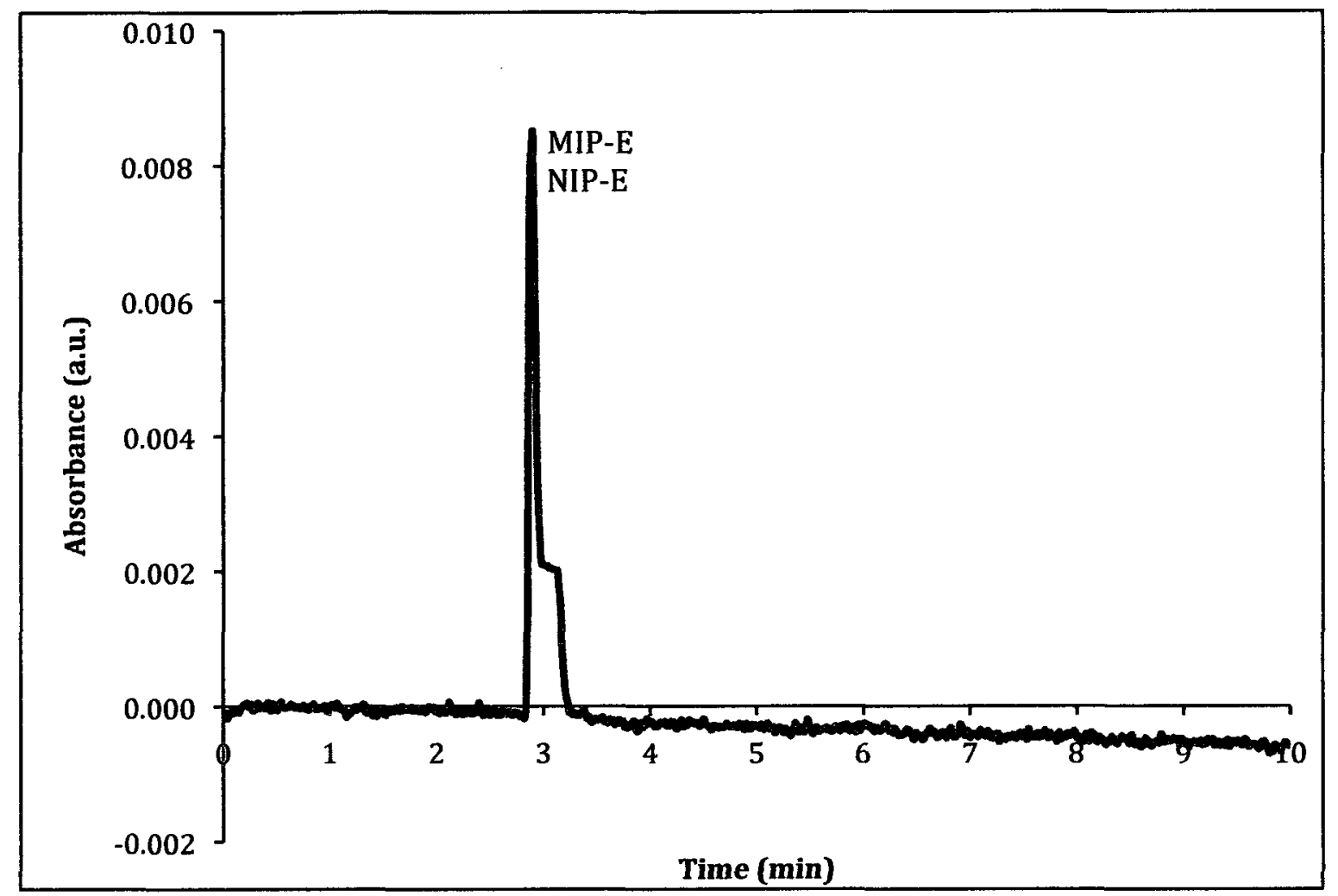

Figure 30 Separation of MIP-E and NIP-E by $\mathrm{CE}$ in $50 \mathrm{mM}$ borate buffer $(\mathrm{pH}=8.5)$.

\subsubsection{Addition of dynamic capillary modifier and separation of MIP and NIP} particles

EOTrol $^{\mathrm{TM}}$ polymer is a line of dynamic coatings which provide unique control over electroosmotic flow (EOF) in capillary. As claimed by the manufacturer, their effects on EOF are nearly independent of $\mathrm{pH}$ and buffer types. Addition of these polymers at a very low concentration to the running buffer (or background electrolyte, BGE) can increase, suppress, or even reverse EOF, in fused silica capillaries (Figure 31) at any buffer pH between 3 and 11. 


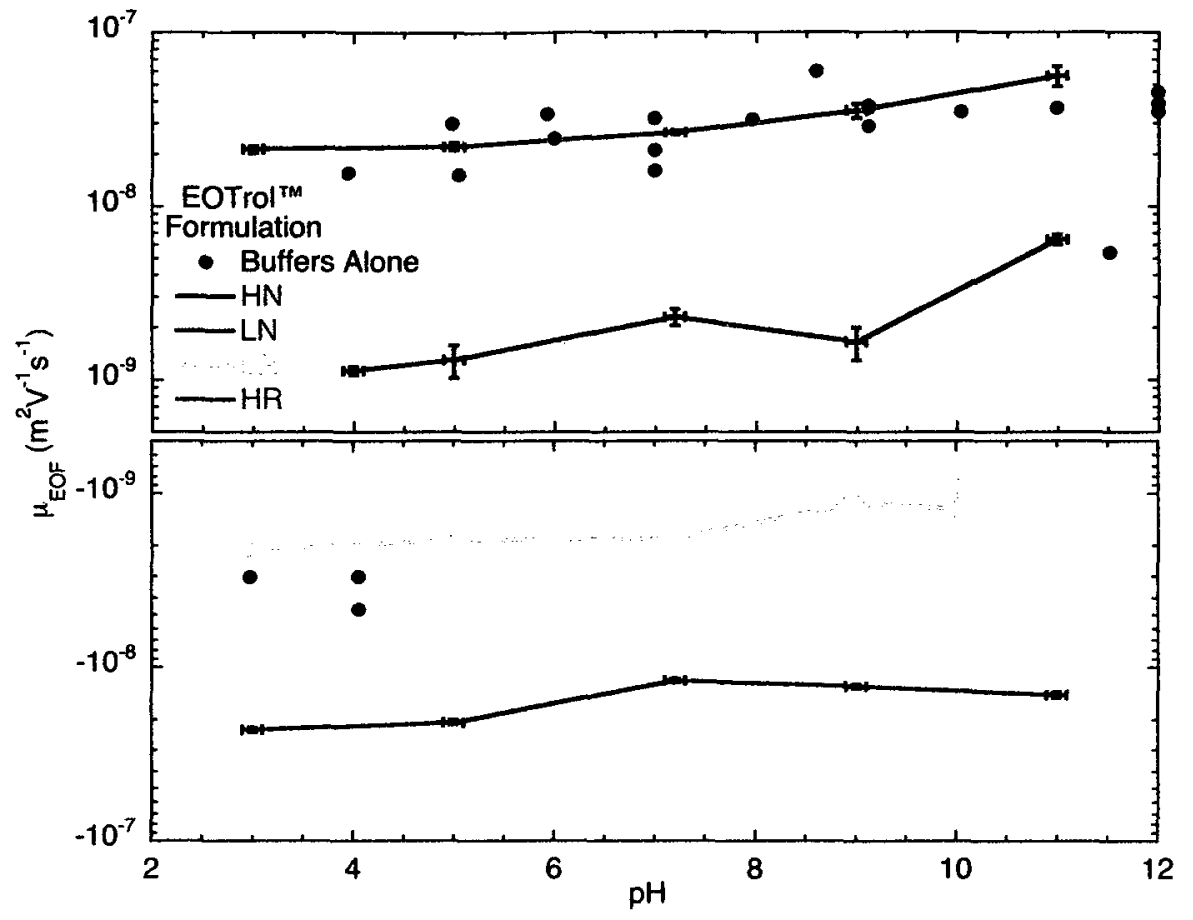

Figure 31 Electroosmotic mobility measured for several EOTrol ${ }^{\mathrm{TM}}$ dynamic coatings as a function of buffer pH in $\mathrm{CZE}$.

As seen in Figure 32, the UV absorption properties of different EOTrols ${ }^{\mathrm{TM}}$ at a concentration of $1.5 \mathrm{mg} / \mathrm{mL}$ in aqueous buffers exhibit no significant absorbance in the near UV region. Their maximum absorbance was observed at $\sim 210 \mathrm{~nm}$, which was close to the wavelength ( $214 \mathrm{~nm}$ ) for the detection of MIP and NIP particles. To minimize the impact of EOTrol ${ }^{\mathrm{TM}}$ absorbance on the UV detection of MIP and NIP particles, a low concentration of EOTrol ${ }^{\mathrm{TM}}$ should be used. As reported by Dabek et al., in their separation of different isomers of benzoic acids, increasing the concentration of EOTrol ${ }^{\mathrm{TM}} \mathrm{LN}$ in the BGE from $0.005 \%$ to $0.15 \%$ (w/v) yielded no significant differences in analysis speed and resolution [71]. So an addition of $0.005 \% \mathrm{EOTrol}^{\mathrm{TM}} \mathrm{LN}$ to the $50 \mathrm{mM}$ borate buffer $(\mathrm{pH}=8.5)$ were chosen to provide an optimal concentration of dynamic modifier in the BGE. 


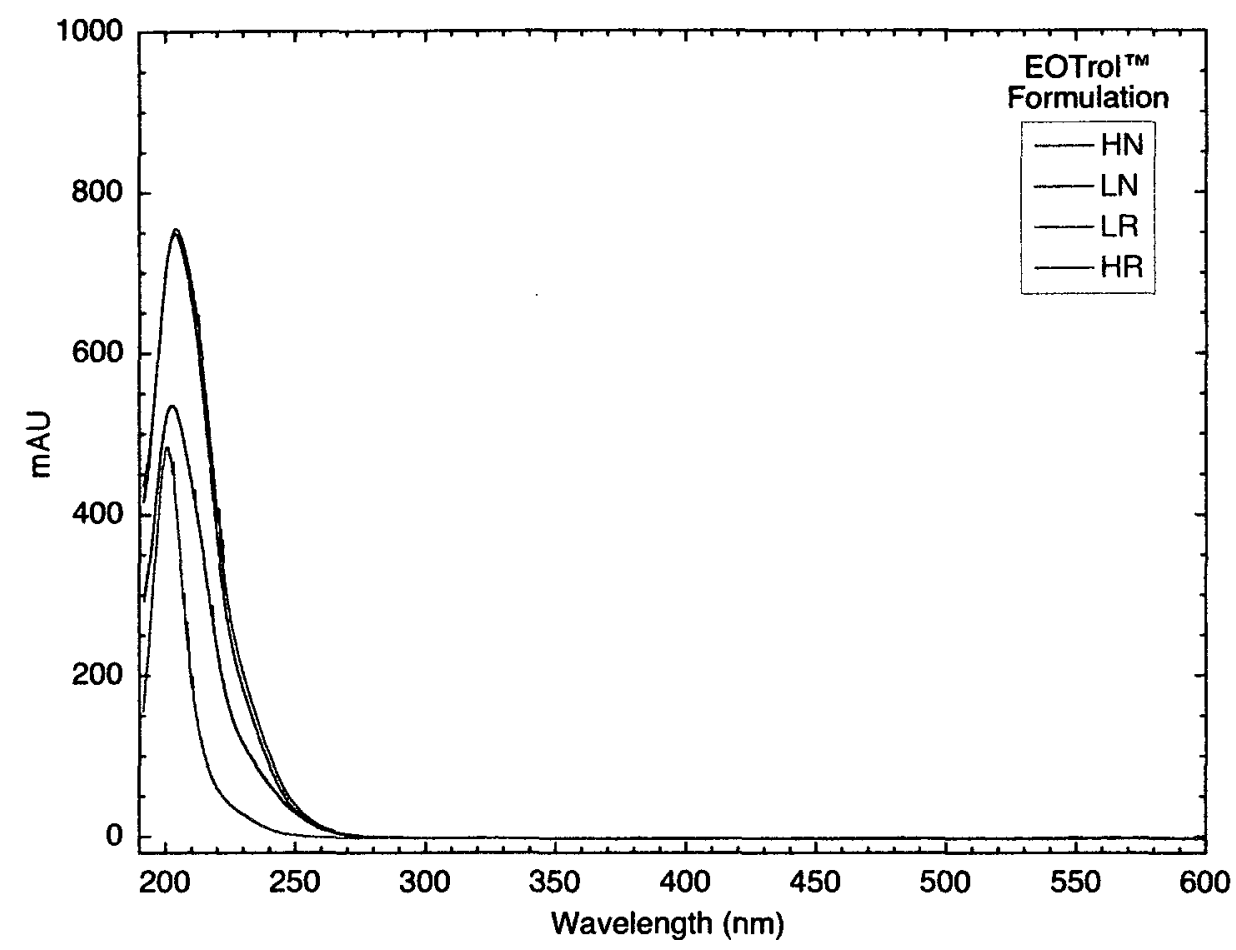

Figure 32 UV-VIS spectra of different EOTrol ${ }^{\mathrm{TM}}$ dynamic coating modifiers in water.

Three CE electropherograms of 1:1 (w/w) mixtures of MIP and NIP particles, obtained without addition of dynamic modifier, were discussed in Section 3.2.1. As all MIPs and NIPs migrated relatively fast (in less than 4 minutes), it was advantageous to control the EOF for a better separation. Thus the effect of EOTrol ${ }^{\mathrm{TM}} \mathrm{LN}$ (as a dynamic modifier) on producing a low EOF $\left(\sim 2 \times 10^{-9} \mathrm{~m}^{2} \mathrm{~V}^{-1} \cdot \mathrm{s}^{-1}\right.$ at $\left.\mathrm{pH} 8.5\right)$ in the forward direction (from sample injection inlet to analytes detection outlet), under normal polarity (anode at inlet and cathode at outlet), for better separation of MIP and NIP particles was investigated. However, after running CE separation on different types of MIPs and NIPs for 40 minutes, using BGE containing $0.005 \% \mathrm{EOTrol}^{\mathrm{TM}} \mathrm{LN}$, no peaks were observed. As discussed in 3.2.1, MIP and NIP particles were negatively charged, and their negative electrophoretic motilities $\left(\mu_{\mathrm{ep}}\right)$ were apparently faster/higher than the low EOF 
mobility $\left(\mu_{\mathrm{eo}}\right)$ in the forward direction. Therefore, $\mu_{\mathrm{app}}=\mu_{\mathrm{eo}}+\mu_{\mathrm{ep}}$ became negative. A negative apparent mobility ( $\mu_{\text {app }}$ ) would explain why the CE separation did not detect the MIP and NIP particles.

Although the EOTrol ${ }^{\mathrm{TM}} \mathrm{LN}$ was originally designed to lower the EOF in the normal polarity, reverse polarity was tested in an attempt to solve the separation problem. Reversing the anode and the cathode allowed the movement of negatively charged particles towards the detector while a low cathodic EOF caused slower migration of all particles (Figures 33 and 34) to afford a better CE separation between MIP and corresponding NIP particles.

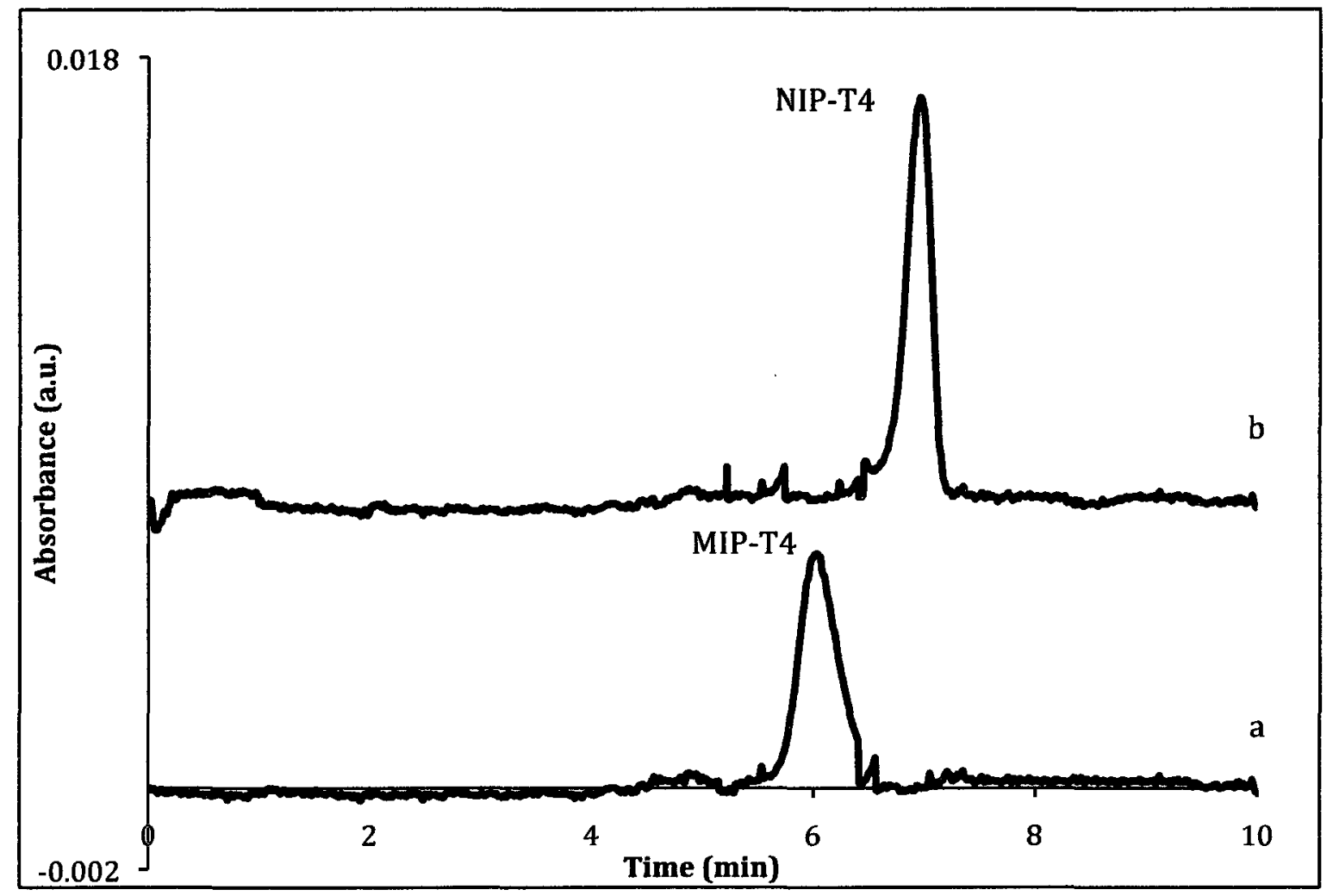

Figure 33 CE electrophoresis of a) MIP-T4 and b) NIP-T4, in $50 \mathrm{mM}$ borate buffer (pH=8.5) containing $0.005 \%$ w/v EOTrol $^{\mathrm{TM}} \mathrm{LN}$, separation voltage $-30 \mathrm{kV}$; detection, UV at $214 \mathrm{~nm}$. 


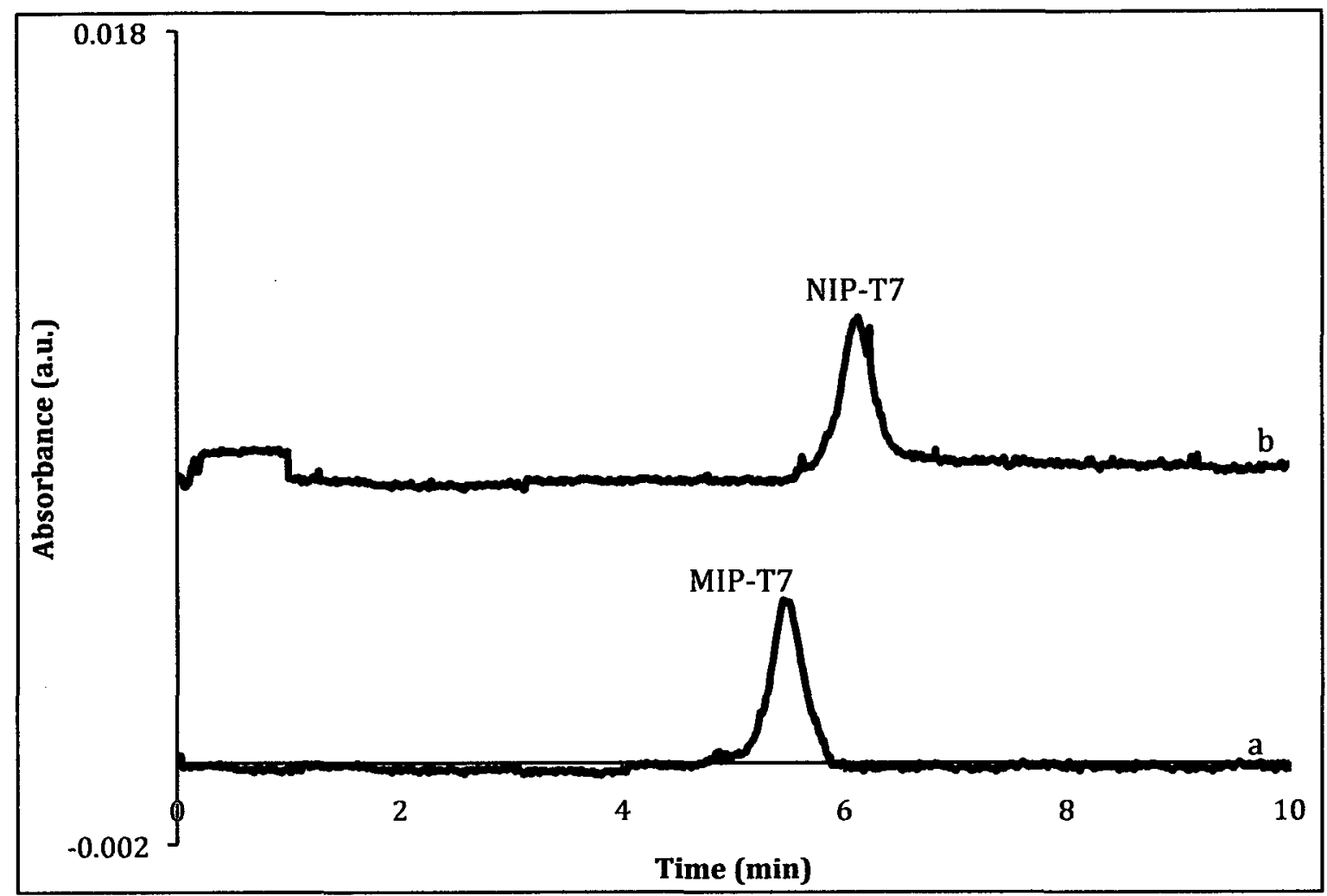

Figure $34 \mathrm{CE}$ electrophoresis of a) MIP-T7 and b) NIP-T7, in $50 \mathrm{mM}$ borate buffer ( $\mathrm{pH}=8.5)$ containing $0.005 \% \mathrm{w} / \mathrm{v}$ EOTroI $^{\mathrm{TM}} \mathrm{LN}$, separation voltage $-30 \mathrm{kV}$; detection, UV at $214 \mathrm{~nm}$.

CE separations of different MIP and NIP particles before and after addition of $0.005 \% \mathrm{EOTrol}^{\mathrm{TM}}$ LN are summarized in Table 10. Table 9 above showed that the mobilities of each pair of MIP and NIP particles, before and after addition of EOTrol ${ }^{\mathrm{TM}} \mathrm{LN}$ as a dynamic coating modifier, were close to each other. However, addition of this modifier significantly improved the resolution of these particles during $\mathrm{CE}$ separation.

Table 10 Resolution of separation of MIP and NIP particles before and after addition of dynamic coating modifier.

\begin{tabular}{|c|c|c|}
\hline \multirow[t]{2}{*}{ Particles } & \multicolumn{2}{|l|}{ Resolution } \\
\hline & Before addition of EOTrol ${ }^{\mathrm{TM}} \mathrm{LN}$ & After addition of EOTrol ${ }^{\mathrm{TM}} \mathrm{LN}$ \\
\hline MIP-E and NIP-E & $\mathrm{n} / \mathrm{a}$ & $\mathrm{n} / \mathrm{a}$ \\
\hline MIP-T4 and NIP-T4 & 0.9 & 1.8 \\
\hline NIP-T7 and NIP-T7 & 0.4 & 1.3 \\
\hline
\end{tabular}




\subsubsection{Characterization of MIP and NIP particles based on the size and charge}

As shown in Figure 35, separation of a 1:1 (w/w) mixture of MIP-T4 and NIP-T4 particles was better after addition of $0.005 \%(\mathrm{w} / \mathrm{v})$ EOTrol ${ }^{\mathrm{TM}} \mathrm{LN}$ to the $50 \mathrm{mM}$ borate buffer ( $\mathrm{pH} 8.5$ ). A study on their binding properties showed that both MIP-T4 and NIP-T4 particles have $150 \pm 15$ $\mu \mathrm{mol} / \mathrm{g}$ of nonspecific binding sites. This suggested nearly the same charge density on the surfaces of these particles. The diameters of MIP-T4 and NIP-T4 particles were determined by using dynamic light scattering (DLS) to be $333 \pm 2$ and $352 \pm 4 \mathrm{~nm}$, respectively. As the charge-tosize ratio is a major factor in the separation of any species by $\mathrm{CE}$, their separation was achieved probably in a size-dependent manner.

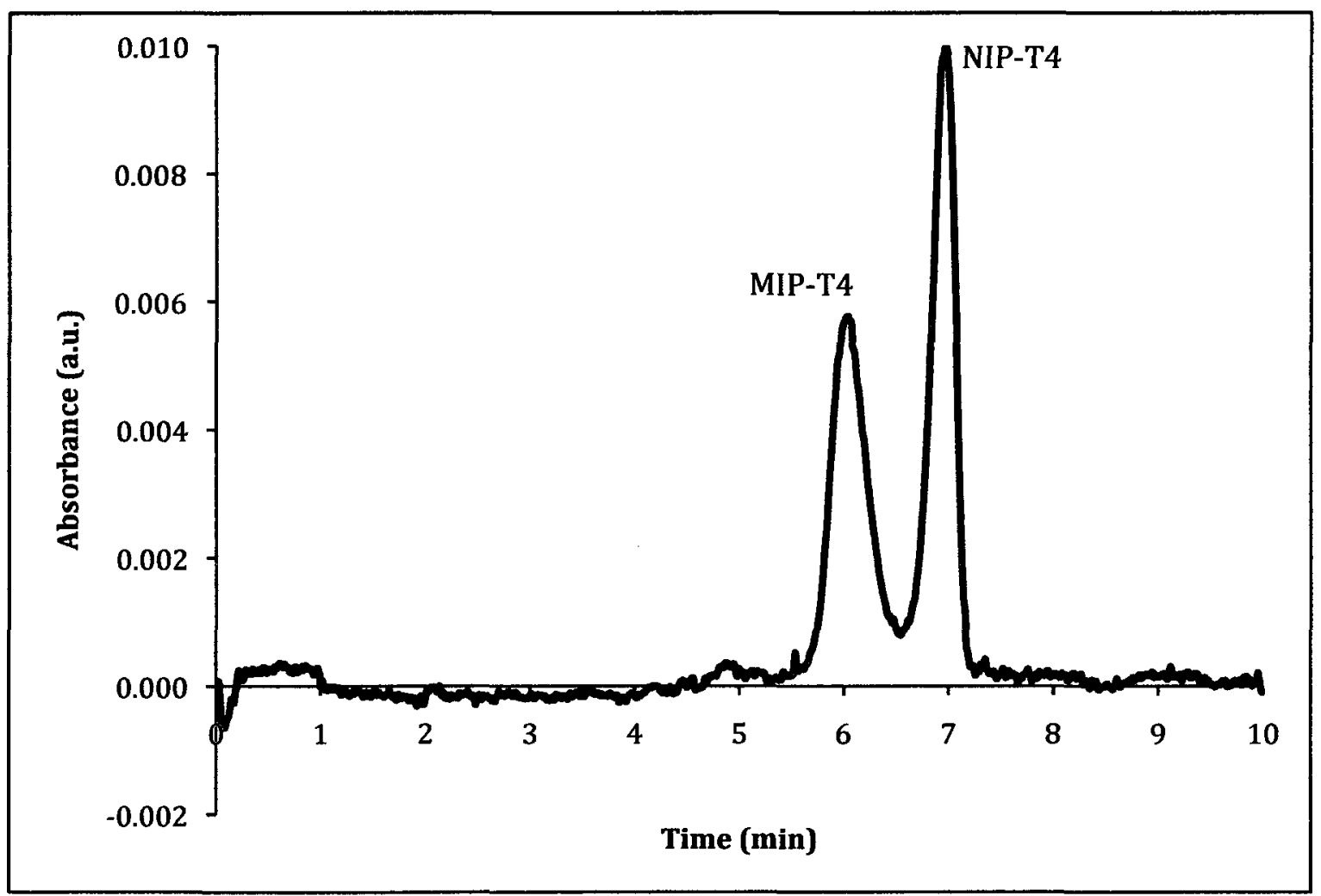

Figure $35 \mathrm{CE}$ electrophoresis of 1:1 mixture of $1 \mathrm{mg} / \mathrm{mL}$ MIP-T4 and NIP-T4 particles, in $50 \mathrm{mM}$ borate buffer (pH=8.5) containing $0.005 \%$ w/v EOTrol ${ }^{\mathrm{TM}} \mathrm{LN}$, under separation voltage of $-30 \mathrm{kV}$, using UV detection at $214 \mathrm{~nm}$. 
Separation of MIP-T7 and NIP-T7 particles is presented in the CE electropherogram of Figure 36. The dynamic diameters of MIP-T7 and NIP-T7 particles were measured to be $282 \pm 2 \mathrm{~nm}$ and $288 \pm 6 \mathrm{~nm}$, respectively. As the difference in size between MIPs and NIPs was not significant, the surface charges on these particles would be dominant. A study of binding properties showed twice as many binding sites on MIP-T7 as on NIP-T7 $(105 \pm 10$ and $55 \pm 9 \mu \mathrm{mol} / \mathrm{g}$, respectively). This suggested a higher number of charged binding sites on the surface of MIP-T7 particles, which could explain their faster migration to result in separation from NIP-T7 particles.

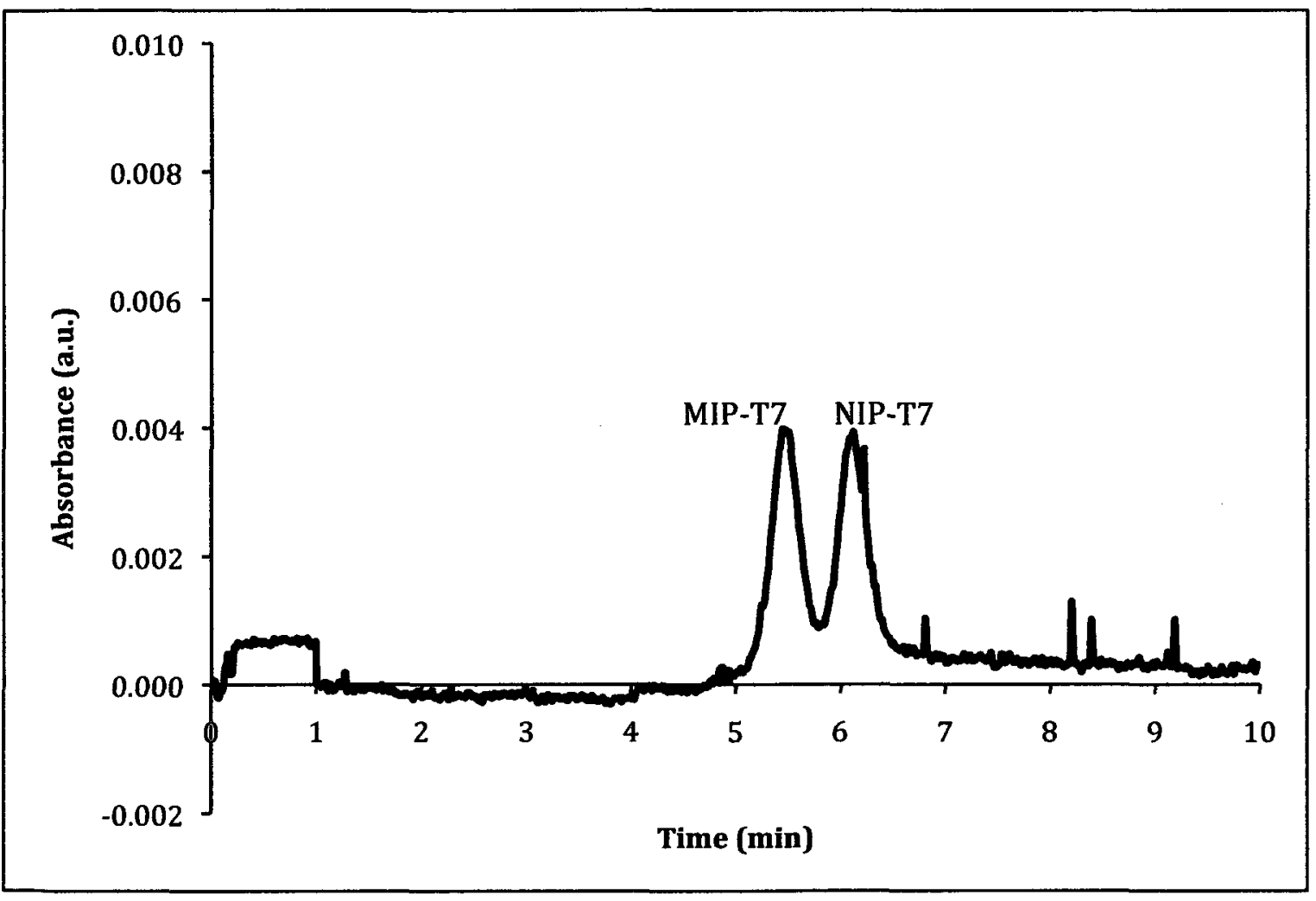

Figure $36 \mathrm{CE}$ electrophoresis of $1: 1 \mathrm{mixture}$ of $1 \mathrm{mg} / \mathrm{mL}$ MIP-T7 and NIP-T7, in $50 \mathrm{mM}$ borate buffer (pH=8.5) containing $0.005 \% \mathrm{w} / \mathrm{v}$ EOTrol ${ }^{\mathrm{TM}} \mathrm{LN}$, separation voltage $-30 \mathrm{kV}$; detection, UV at $24 \mathrm{~nm}$. 


\subsubsection{Nonspecific binding sites of MIP and NIP particles}

The classical determination of nonspecific binding sites on the surface of MIPs and NIPs is a Scatchard plot. This determination is time consuming and requires much chemical and particles. To quickly compare the nonspecific binding capacities of MIP and NIP particles, information about the sizes and mobilities of these particles could be useful. Figure 37 shows a typical electropherogram of a 1:1 (w/w) mixture of MIP-E and NIP-E particles using the BGE containing $0.005 \% \mathrm{w} / \mathrm{v}$ EOTrol ${ }^{\mathrm{TM}} \mathrm{LN}$. Their dynamic diameters were determined to be $578 \pm 23$ $\mathrm{nm}$ and $366 \pm 8 \mathrm{~nm}$, respectively, by DLS. Although there was a significant difference in size between these two types of particles, no CE separation took place, which suggested their equal charge-to-size ratios. When the diameter of MIP-E particles was 50\% larger than that for NIP-E particles, a larger surface charge density for the MIP-E particles would be proposed. As a result, a larger nonspecific binding capacity of MIP-E particles was expected. This was confirmed by a study of the binding properties of MIP-E and NIP-E particles, to be $57 \pm 9$ and $37 \pm 8 \mu \mathrm{mol} / \mathrm{g}$ respectively (Table 5). 


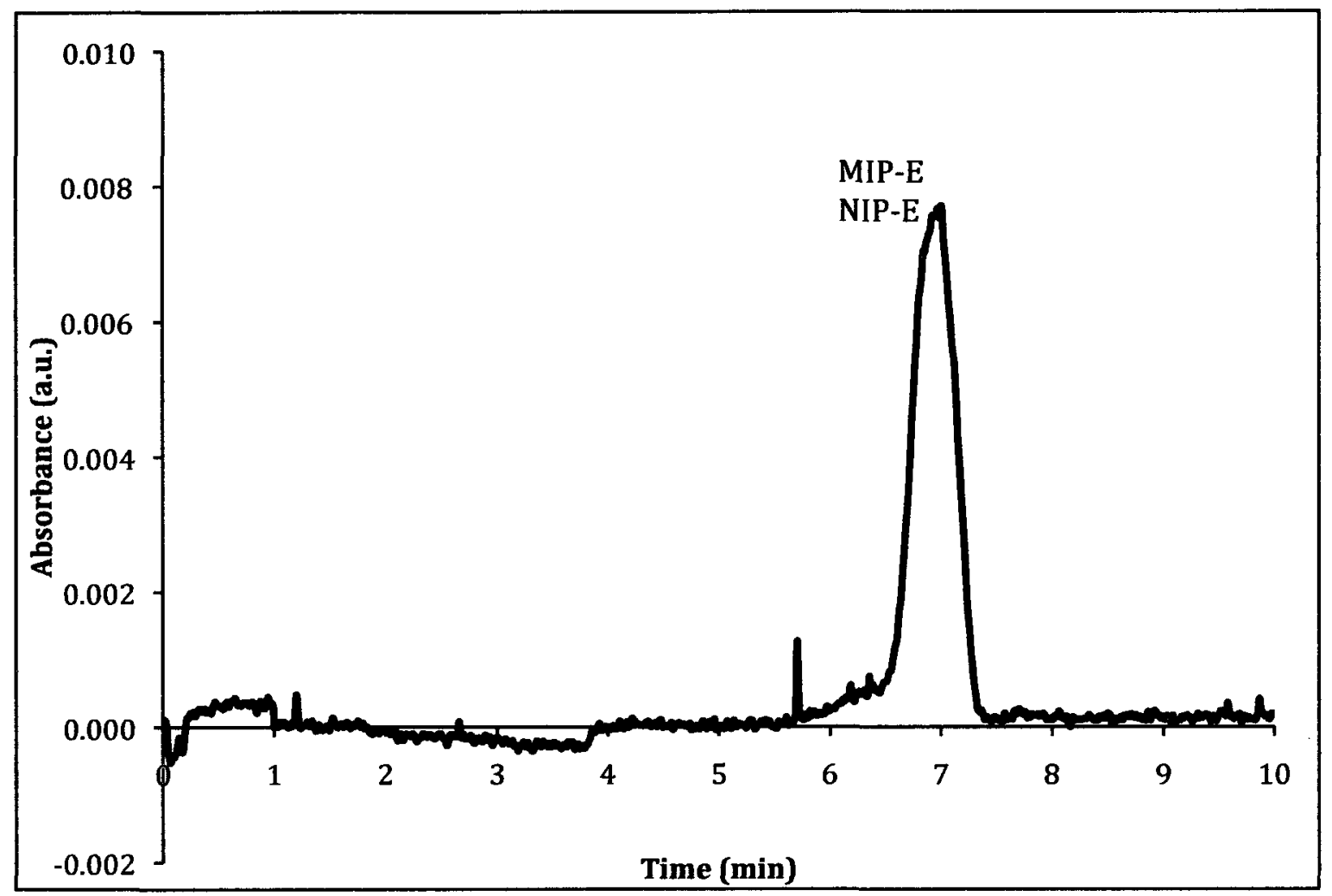

Figure $37 \mathrm{CE}$ of $1 \mathrm{mg} / \mathrm{mL}$ MIP-E and $1 \mathrm{mg} / \mathrm{mL}$ NIP-E, in $50 \mathrm{mM}$ borate buffer $(\mathrm{pH}=8.5)$ containing $0.005 \% \mathrm{w} / \mathrm{v}$ EOTrol $^{\mathrm{TM}} \mathrm{LN}$; separation voltage: $-30 \mathrm{kV}$; UV detection at $24 \mathrm{~nm}$.

\subsubsection{Kinetic study of NIP particles binding with bisphenol A}

Binding with MIP or NIP particles would require time for diffusion of analyte molecules into the macroporous polymer matrix of imprinted cavities. To determine the shortest incubation time for reaching equilibrium, binding with MIPs or NIPs over 2-60 min was studied. Centrifugation before HPLC-FD analysis of the supernatant, however, did not allow testing of any incubation time less than 2 minutes. As a result, investigation of alternate methods was essential. Due to the suitability of theses submicron particle sizes for $\mathrm{CE}$, the kinetics of nonspecific BPA binding on NIP-T4 and NIP-T7 particles could be investigated without centrifugation. Figure 38 shows the CE electropherogram of BPA before and after binding on NIP-T4. 


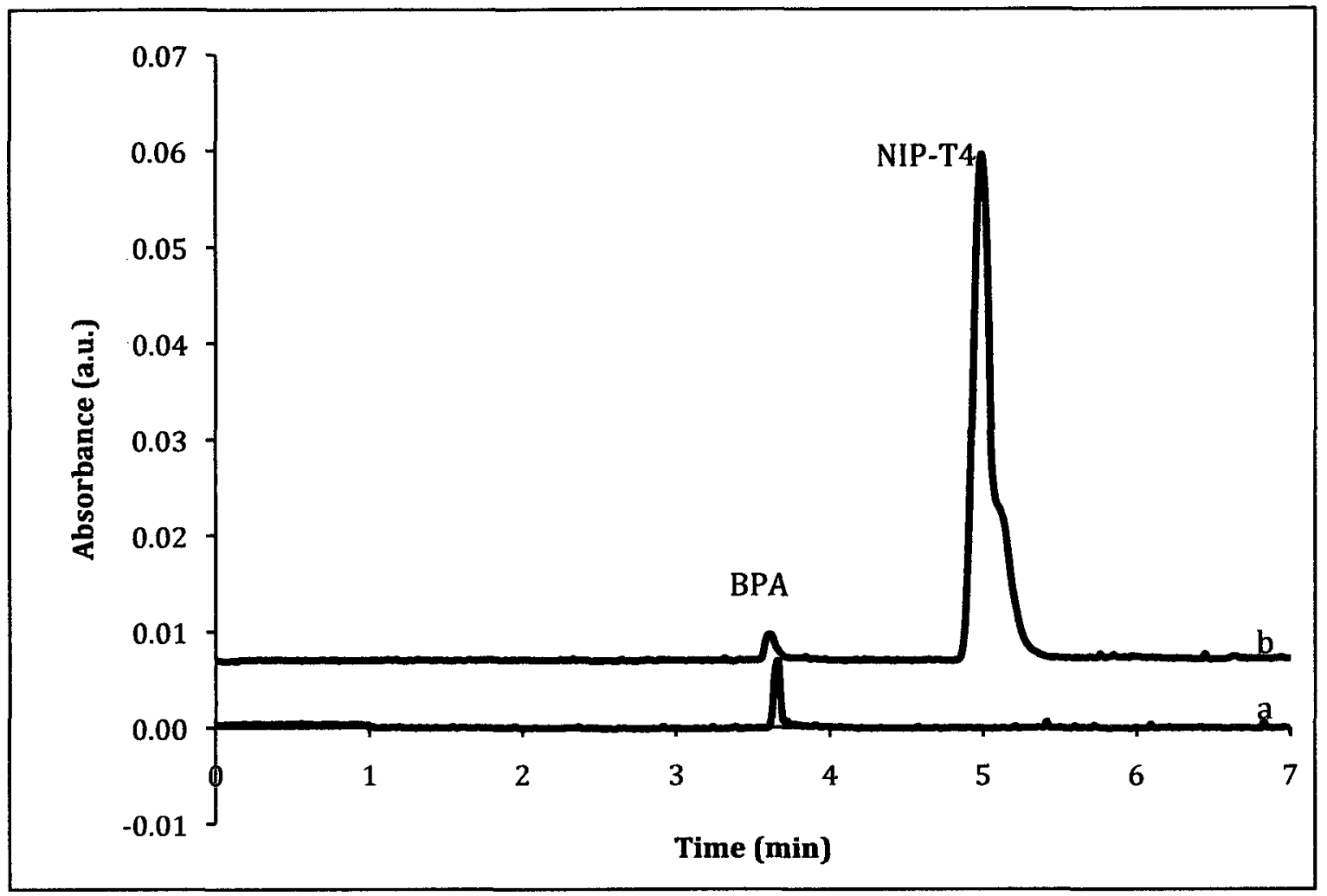

Figure 38 CE electropherograms of (a) $20 \mu \mathrm{g} / \mathrm{mL} \mathrm{BPA}$, and (b) $1 \mathrm{mg} / \mathrm{mL}$ of NIP-T4 in $20 \mu \mathrm{g} / \mathrm{mL} \mathrm{BPA}$ after $29 \mathrm{~s}$ of incubation.

As shown in Figure 39, the amount of BPA bound non-specifically onto NIP-T4 increased with time. The bound BPA increased to $52 \pm 4 \%$ as the incubation time went up to $45 \mathrm{~s}$ and beyond. This indicates that $45 \mathrm{~s}$ of incubation were sufficient time to bind BPA with NIP-T4 particles under equilibrium conditions. The equilibrium constant (conditional upon $1 \mathrm{mg} / \mathrm{mL}$ of NIP-T4 added) can be expressed as:

$$
K^{\prime} \text { non }- \text { specific }=\frac{[B P A] \text { initial }-[B P A] \text { eq }}{[B P A] \text { eq }}=\frac{\% \text { binding }}{1-\% \text { binding }}=\frac{0.54}{1-0.54}=1.2
$$




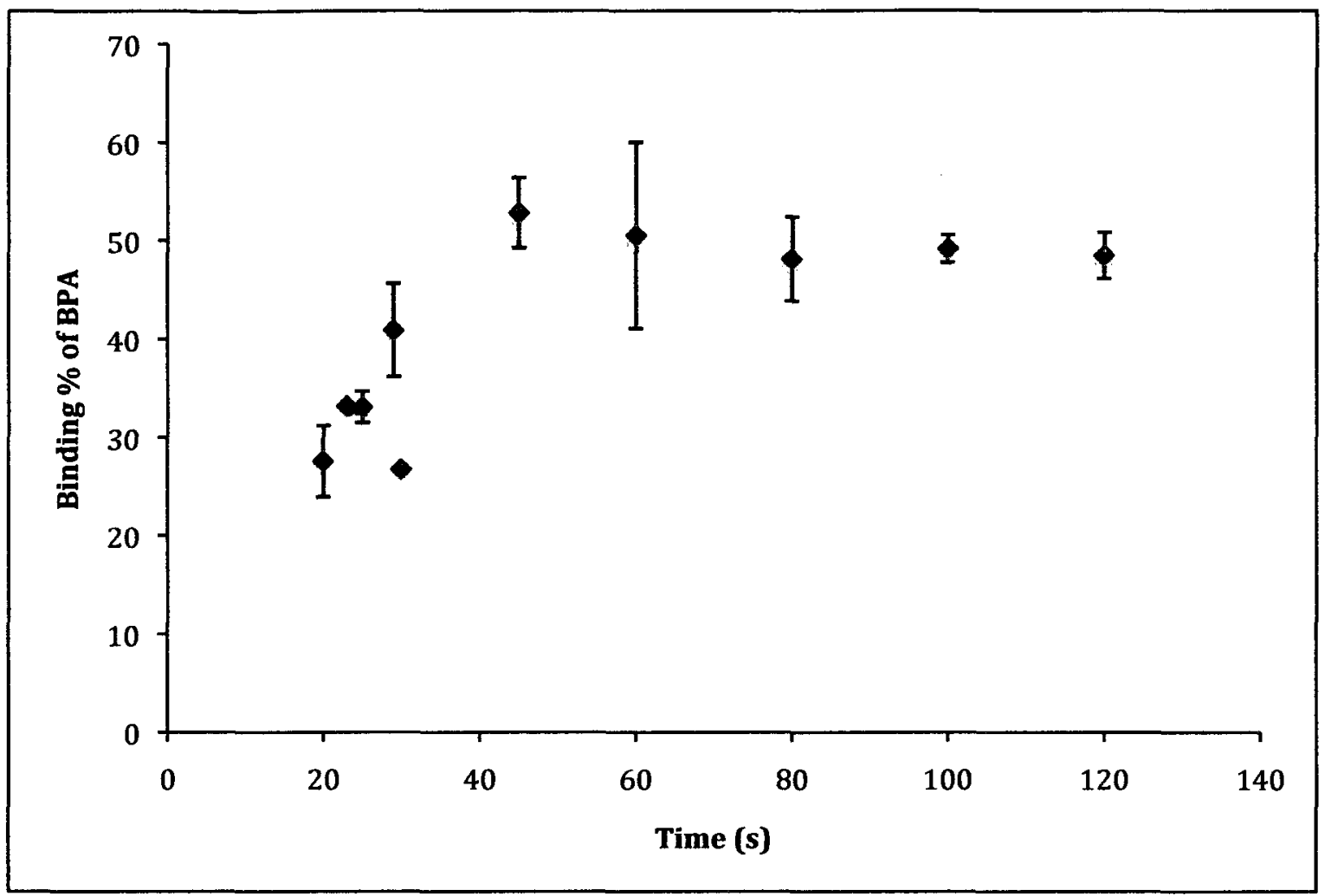

Figure $39 \%$ binding of BPA after adding $1 \mathrm{mg} / \mathrm{mL} \mathrm{NIP-T4}$ into $20 \mu \mathrm{g} / \mathrm{mL}$ of BPA for $20-120 \mathrm{~s}$.

Non-specific binding of BPA onto NIP-T7 was next tested under the same experimental conditions as for NIP-T4. The result in Figure 40 indicated that even before $45 \mathrm{~s}$ of incubation, $48 \pm 4 \%$ of BPA were bound on NIP-T7 at equilibrium. Faster binding of BPA onto NIP-T7, as compared to NIP-T4, could be explained by the smaller NIP-T7 particles that provided a larger specific surface area (or surface area per unit mass). The equilibrium constant (conditional upon $1 \mathrm{mg} / \mathrm{mL}$ of NIP-T7 added) can be calculated as:

$$
K^{\prime} \text { non }- \text { specific }=\frac{[B P A] \text { initial }-[B P A] \text { eq }}{[B P A] \text { eq }}=\frac{\% \text { binding }}{1-\% \text { binding }}=\frac{0.48}{1-0.48}=0.9
$$

It is important to note that the discussed equilibrium constants are unit less parameter to compare the non-specific binding of BPA onto the NIP-T4 and NIP-T7 particles and they are not 
comparable with association constant of particles that are calculated for the different types of MIPs and NIPs using scatchard plots.

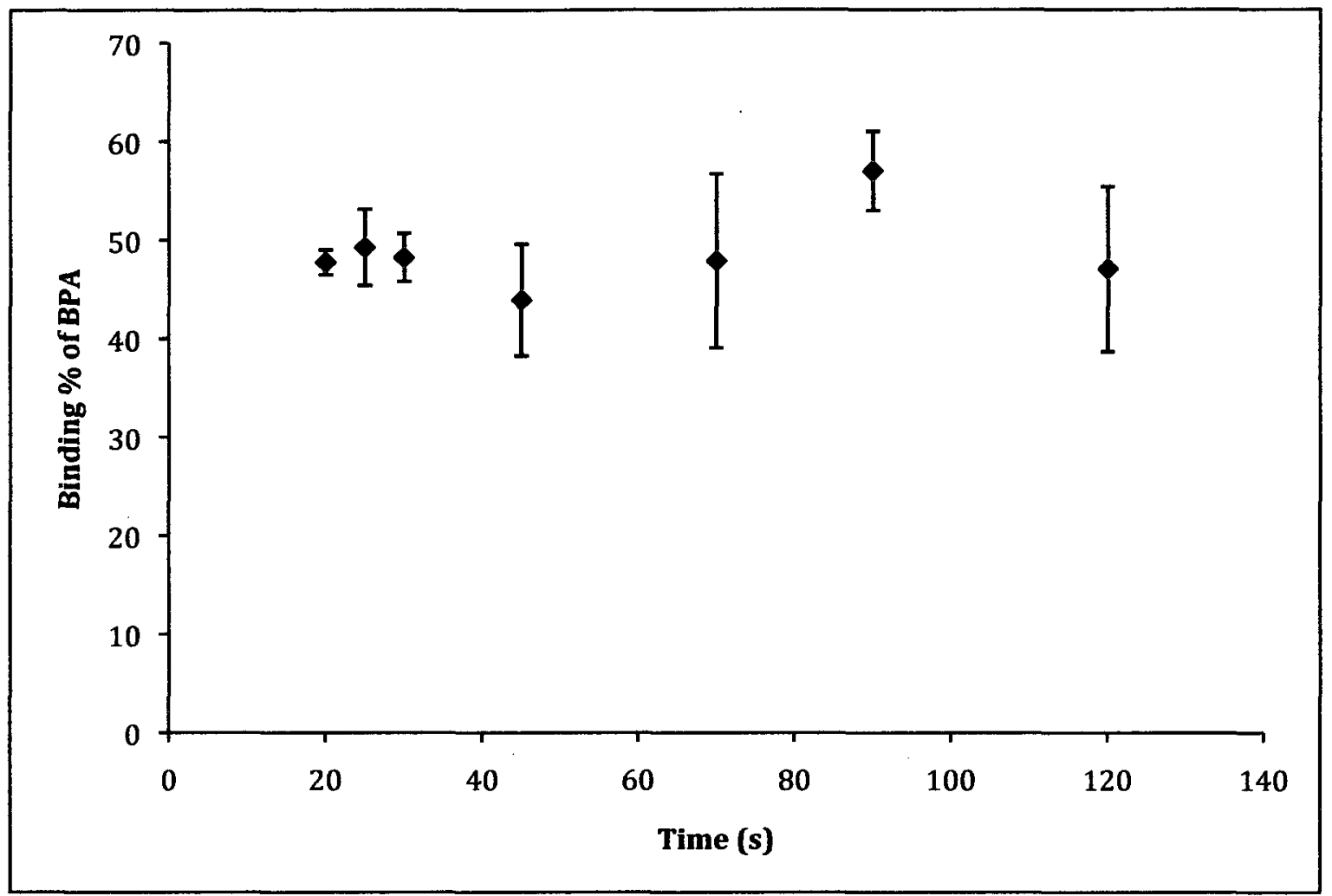

Figure $40 \%$ binding of BPA after adding $1 \mathrm{mg} / \mathrm{mL} \mathrm{NIP-T7}$ into $20 \mu \mathrm{g} / \mathrm{mL}$ of BPA for $20-120 \mathrm{~s}$.

Intuitively, the submicron dimension of particles ensured rapid diffusion of BPA molecules throughout the polymer structure to attain binding equilibrium. It is clearly demonstrated that electrophoretic separation of the co-injected BPA and NIPs set the lower limit at $\sim 20 \mathrm{~s}$ for a permissible incubation time. This lower limit was dictated by the $15 \mathrm{~s}$ required for instrumental operation to make a new injection of the BPA/NIP particles mixture from a sample vial. There was no need to remove the particles (by centrifugation or filtration) prior to $\mathrm{CE}$ analysis. This is an advantage which is not afforded by HPLC or LC-MS. Moreover, a significant difference in mobility between BPA and NIPs was observed. Such difference guaranteed no overlap between the two peaks, to ensure accuracy in their area measurements. A later experiment indicated that 
binding BPA over NIP particles had no effect on the mobility of these particles. Hence, these CE peaks would not overlap with each other. (Figure 41)

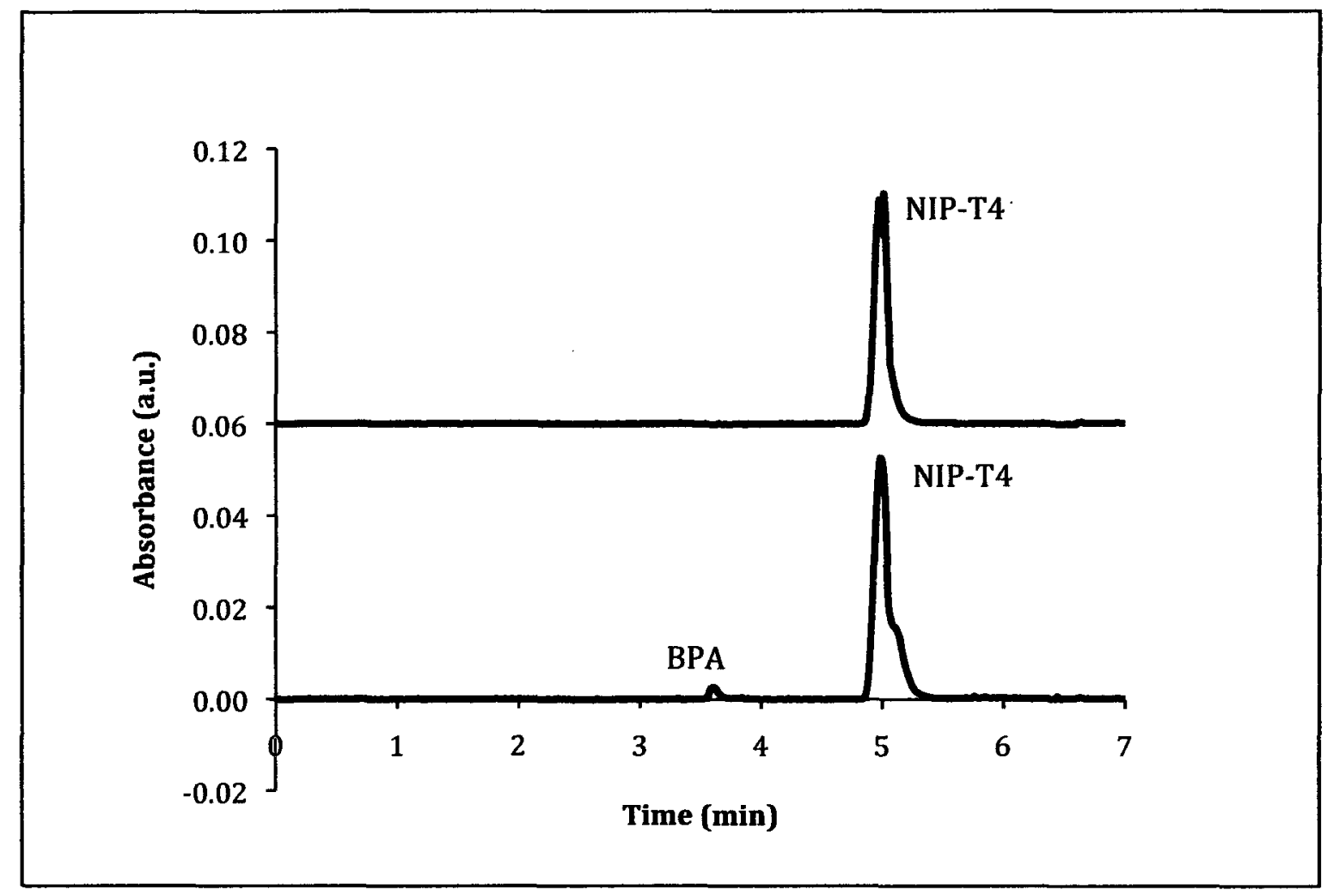

Figure 41 CE electropherograms of NIP-T 4 before $(-)$ and after $(-)$ binding with BPA.

\subsubsection{Selectivity of particles using CE}

\subsubsection{Selectivity of MIP-T4 particles on E2 in presence of BPA}

The selectivity of MIP-T4 particles in comparison with NIP-T4 particles was studied. Binding efficiencies for a constant amount of BPA, in the presence of various E2 concentrations, on MIPT4 and NIP-T4 particles were plotted in Figures 42 and 43. 


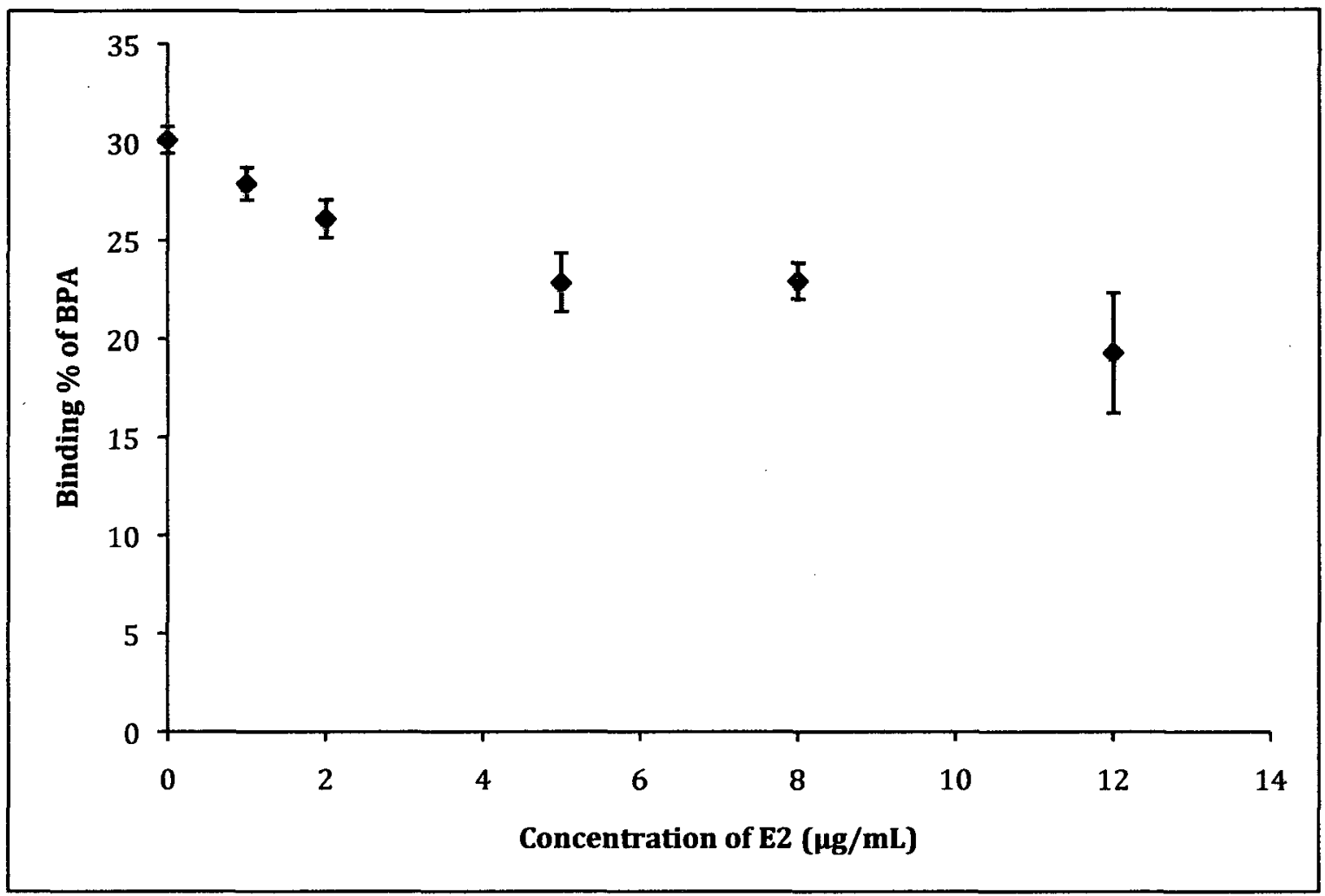

Figure 42 Binding efficiency of BPA on MIP-T4 particles in presence of different concentrations of E2. Concentration of BPA was $20 \mu \mathrm{g} / \mathrm{mL}$.

The result indicated that, when there was no $\mathrm{E} 2$ in the mixture, $30 \pm 1 \%$ of BPA (or $27 \pm 1 \mu \mathrm{mol} / \mathrm{g}$ ) bound nonspecifically on the MIP-T4. Figure 42 shows the change in binding $\%$ of BPA on MIP-T4 particles for various E2 concentrations. For instance, $1 \mu \mathrm{g} / \mathrm{mL}$ E2 reduces the binding efficiency to $27 \pm 1 \%$ (or $24 \pm 1 \mu \mathrm{mol} / \mathrm{g}$ ). As observed in Table 5 , the specific binding capacity of MIP-T4 was measured to be $33 \pm 7 \mu \mathrm{mol} / \mathrm{g}$. Based on the results summarized in Table 11 , it can be stated that the E2 molecules were first attracted to the high-affinity specific binding sites. Therefore, BPA subsequently bound nonspecifically on the remaining high-affinity binding sites and the low-affinity binding sites. (Note that high and low affinities are defined for E2, not for BPA). When E2 concentration increased to $12 \mu \mathrm{g} / \mathrm{mL}$, the amount of E2 $(44 \mu \mathrm{mol} / \mathrm{g})$ is larger than all available specific binding sites of MIP-T4 $(33 \pm 7 \mu \mathrm{mol} / \mathrm{g})$. About $33 \mu \mathrm{mol}$ of E2 molecules saturated the specific binding sites and the remaining E2 $(11 \pm 7 \mu \mathrm{mol})$ bound onto 
nonspecific binding sites. All BPA molecules had to compete for the low-affinity binding sites, which resulted in a drop in \% binding for BPA.

Table 11 Amounts of bound BPA on MIP-T4 and NIP-T4 particles in presence of E2.

\begin{tabular}{llll}
\hline & & MIP-T4 & NIP-T4 \\
E2 in mixture $(\mu \mathrm{g} / \mathrm{mL})$ & Accessible E2 $(\mu \mathrm{mol} / \mathrm{g})$ & Bound PBA $(\mu \mathrm{mol} / \mathrm{g})$ \\
\hline 0 & 0 & $27 \pm 1$ & $19 \pm 1$ \\
1 & 3.7 & $24 \pm 1$ & $19 \pm 1$ \\
2 & 7.3 & $23 \pm 1$ & $18 \pm 2$ \\
5 & 18.4 & $20 \pm 1$ & $18 \pm 1$ \\
8 & 29.4 & $20 \pm 1$ & $20 \pm 1$ \\
12 & 44.0 & $17 \pm 3$ & $18 \pm 2$ \\
\hline
\end{tabular}

As a comparison, the above experiment was repeated using NIP-T4. Figure 43 summarized, that in the presence of various E2 concentrations, no significant changes in binding efficiencies for BPA on NIP-T4 particles were observed. The constant binding trend for BPA on NIP-T4 particles, in comparison with MIP-T4, can be explained by the absence of high-affinity (specific) binding sites on NIP-T4 particles. 


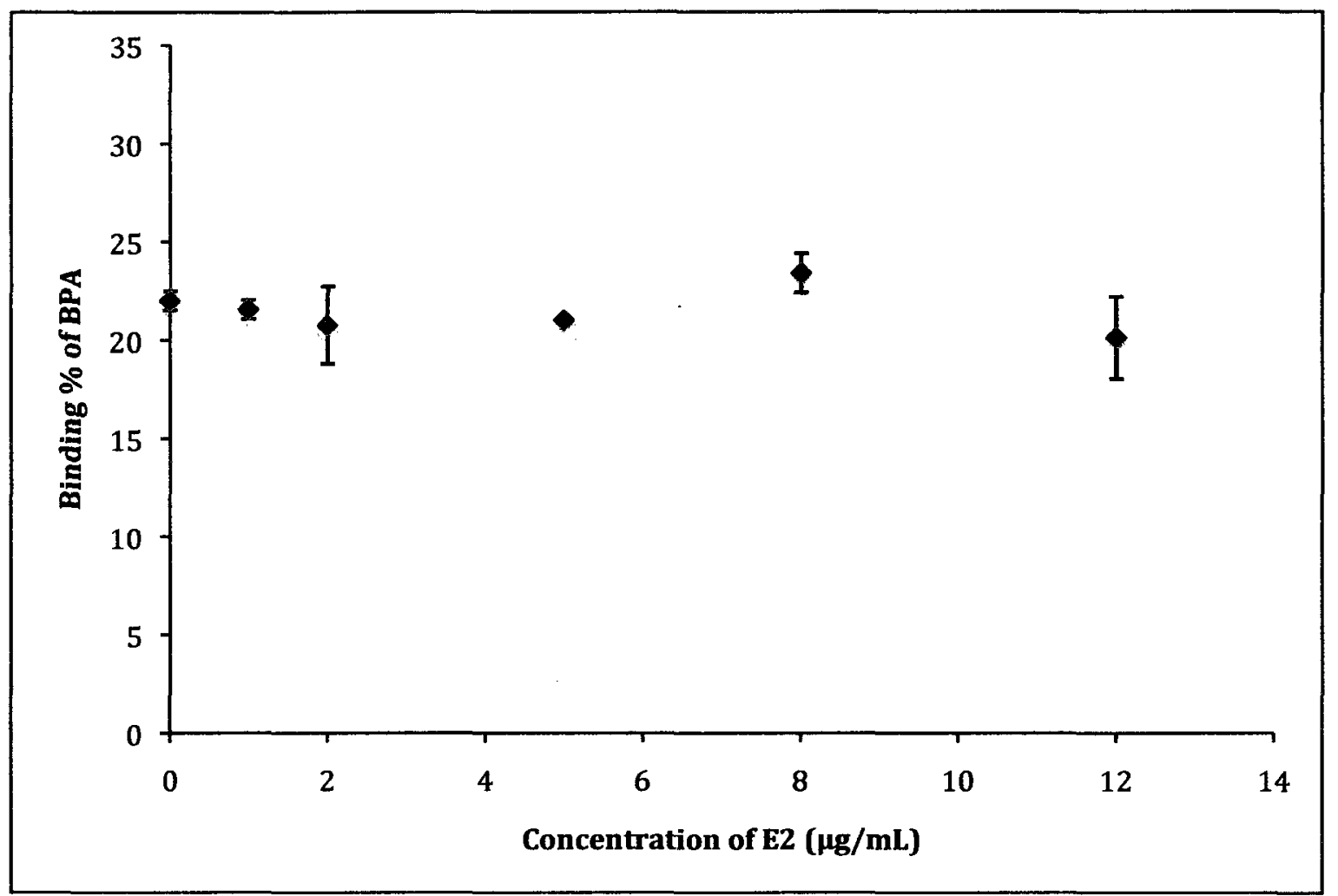

Figure 43 Binding efficiency of BPA on NIP-T4 particles in presence of different concentrations of E2. Concentration of BPA was $20 \mu \mathrm{g} / \mathrm{mL}$.

Another experiment was based on the total concentrations for BPA and E2 (mentioned in the earlier experiment). Increasing amounts of BPA were added to $20 \mu \mathrm{g} / \mathrm{mL}$ BPA, making the total concentration as high as $32 \mu \mathrm{g} / \mathrm{mL}$ BPA. As seen in Figure 44, despite the increasing concentration of BPA, the binding efficiency on MIP-T4 (and NIP-T4) particles remained constant. This could explain that the decrease in binding efficiency of BPA on MIP-T4 particles (in Fig. 41) was due to higher affinity of E2 for these particles during a competitive binding. In other words, the high concentration of analyte had no effect on diminishing the binding efficiency (which was governed by the magnitude of 1.2 for $\mathrm{K}_{\text {non-specific }}$ as discussed on 3.2.3). 


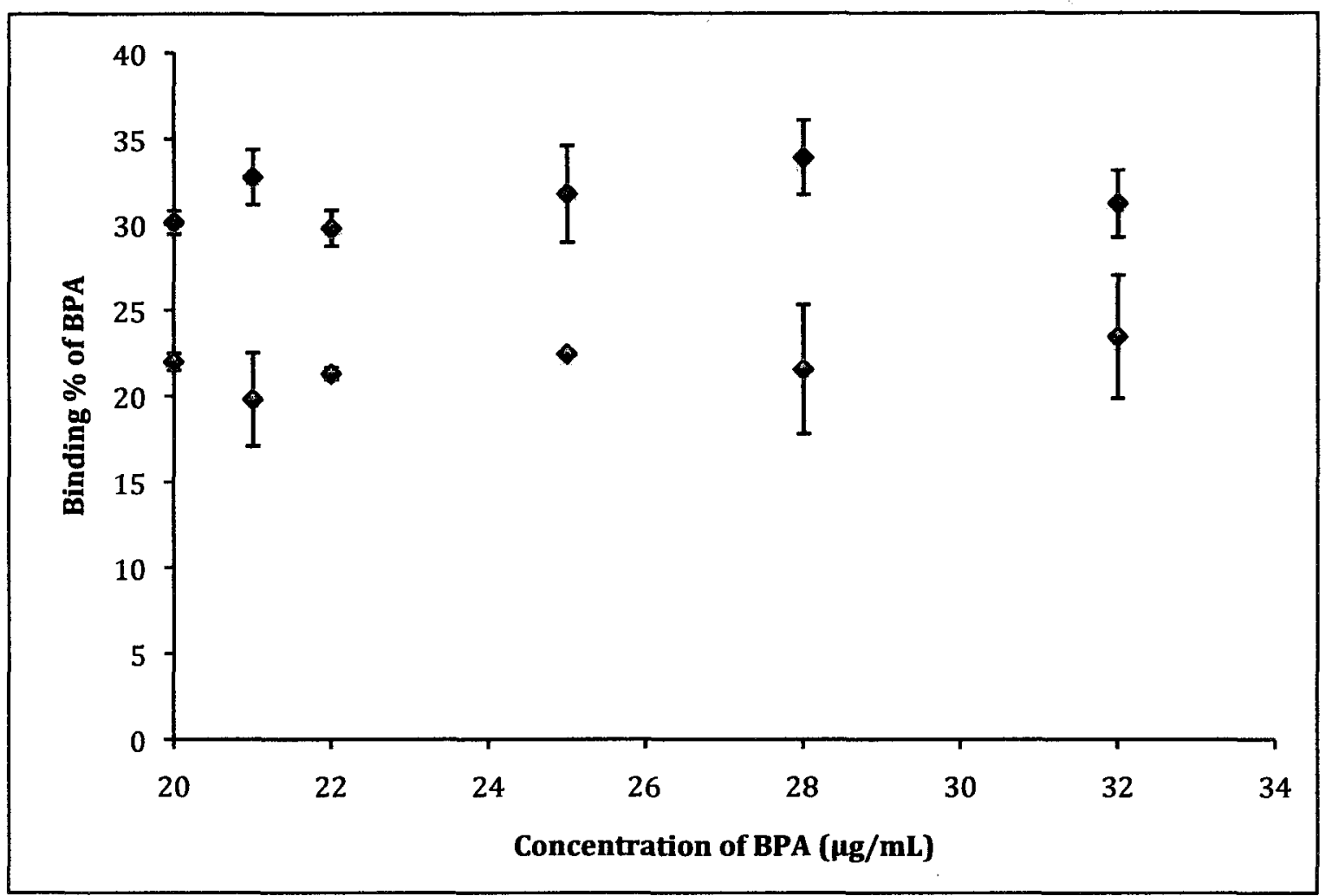

Figure 44 Binding efficiency of BPA MIP-T4 $(\bullet)$ and NIP-T4 $(\bullet)$ in presence of different concentrations of BPA.

\subsubsection{Selectivity of MIP-T7 particles for E2 in presence of BPA}

Selectivity of the MIP-T7 (in comparison to NIP-T7 particles) was tested by determining the binding efficiency of BPA versus different E2 concentrations. In the absence of E2, the binding efficiency of BPA on MIP-T7 particles was determined to be $47 \pm 1 \%$, which was approximately 17\% higher than BPA on MIP-T4 particles. This could be due to a large number of high-affinity binding sites on MIP-T7 particles $(43 \pm 8 \mu \mathrm{mol} / \mathrm{g})$. As seen in Figure 45 , when the E2 concentration was increased from 0 up to $5 \mu \mathrm{g} / \mathrm{mL}$, the binding efficiency of BPA on MIP-T7 particles decreased. However, in the presence of 8 and $12 \mu \mathrm{g} / \mathrm{mL} \mathrm{E} 2$, the binding efficiency of BPA onto the MIP-T7 decreased to a relatively constant $25 \pm 2 \%$. Table 12 shows from 0 up to 5 $\mu \mathrm{g} / \mathrm{mL}$ E2 (in mixture), the sum of available (or added) E2 and bound BPA is smaller or close to 
specific binding capacity of MIP-T7 particles $(43 \pm 8 \mu \mathrm{mol} / \mathrm{g})$. This could indicate that both E2 and BPA were attracted to the high-affinity binding sites in the MIP-T7 particles. As the cavities are specifically imprinted for E2, the majority of the available E2 were bound onto the highaffinity sites. The remaining high-affinity binding sites were occupied by BPA. Further on, in the presence of 8 and $12 \mu \mathrm{g} / \mathrm{mL}$ of E2, the constant binding efficiency of BPA onto the MIP-T7 particles indicated that, as most of the high-affinity binding sites were occupied by E2, BPA were bound onto the non-specific sites. Based on large availability of non-specific binding sites on MP-T7 $(105 \pm 10 \mu \mathrm{mol} / \mathrm{g})$, constant binding efficiency of BPA were maintained. All of the above discussion strongly suggests that analytes, target or not, were attracted to the specific binding sites first.

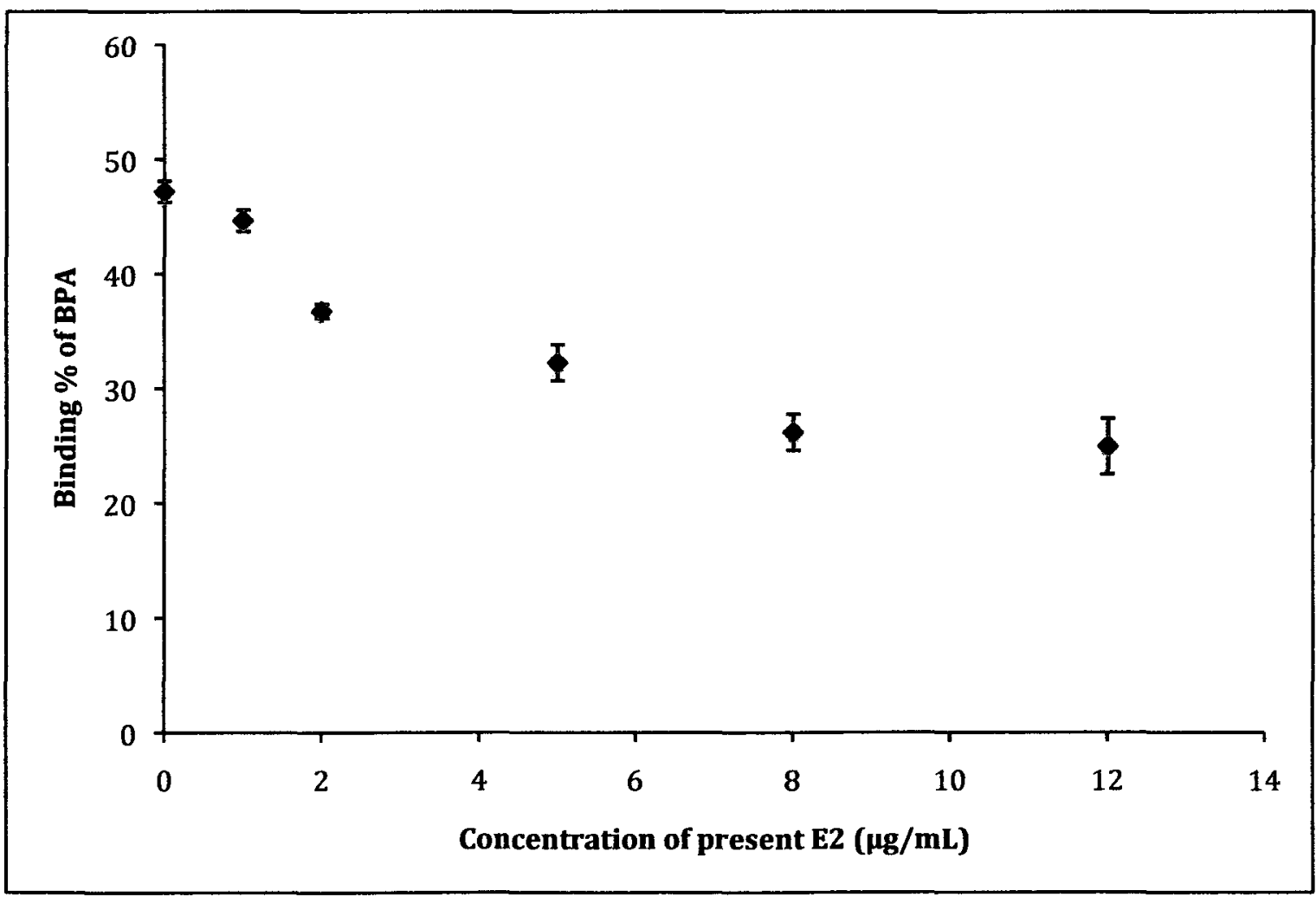

Figure 45 Binding efficiency of BPA on MIP-T7 particles in presence of different concentrations of E2. 
Table 12 Amount of bound BPA on MIP-T7 and NIP-T7 particles in presence of E2.

\begin{tabular}{llll}
\hline E2 in mixture $(\mu \mathrm{g} / \mathrm{mL})$ & Available E2 $(\mu \mathrm{mol} / \mathrm{g})$ & \multicolumn{1}{l}{ MIP-T7 } & NIP-T7 \\
\hline 0 & 0 & $41 \pm 1$ & $41 \pm 1$ \\
1 & 3.7 & $39 \pm 1$ & $43 \pm 1$ \\
2 & 7.3 & $32 \pm 1$ & $42 \pm 2$ \\
5 & 18.4 & $28 \pm 1$ & $37 \pm 4$ \\
8 & 29.4 & $23 \pm 1$ & $26 \pm 15$ \\
12 & 44.0 & $22 \pm 2$ & $23 \pm 19$ \\
\hline
\end{tabular}

As seen in Figure 46, the binding efficiency of BPA onto NIP-T7 particles decreased when the concentration of E2 was increased. Table 12 shows that with the addition of $5 \mu \mathrm{g} / \mathrm{mL}$ of E2 and higher, the total amount of available E2 and bound BPA exceeded the binding capacities of NIPT7 particles $(55 \pm 9 \mu \mathrm{mol} / \mathrm{g})$. As only a limited number of non-specific binding sites were available on the NIP-T7 particles, BPA and E2 competed equally and non-selectively for binding with them, which resulted in a considerable variation of binding $\%$ and hence the large error bars. At the upper limit of each error bar, BPA won over E2, and vice versa at the lower limit. 


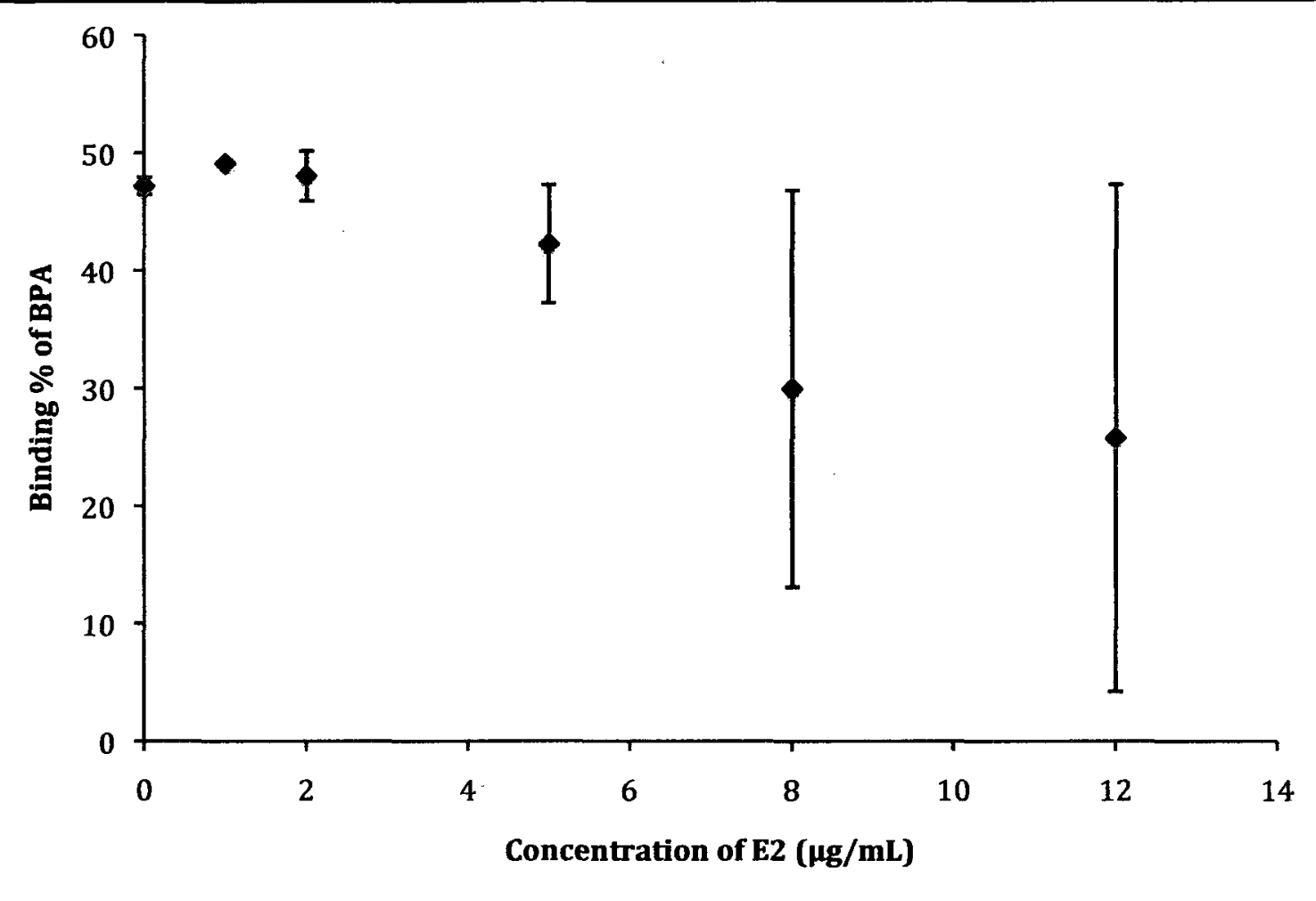

Figure 46 Binding efficiency of BPA on NIP-T7 particles in presence of different concentrations of E2.

Upon the addition of BPA (Figure 47), when the concentration of BPA increased, both types of particles presented the same trend in binding efficiency of BPA because there was no more highaffinity binding sites available for BPA. Table 13 shows that the amount of BPA bound (onto the NIP-T7 particles) was nearly limited by the binding capacity $(55 \pm 9 \mu \mathrm{mol} / \mathrm{g})$.

Overall, the selectivity study on MIPs indicated that both MIP-T4 and MIP-T7 particles were selective for the target (E2). However, the selectivity study on NIP-T4 and NIP-T7 particles showed no difference for binding with E2 or BPA. This led us to continue with sequence addition of BPA and E2 only on MIP particles in the following sections. 


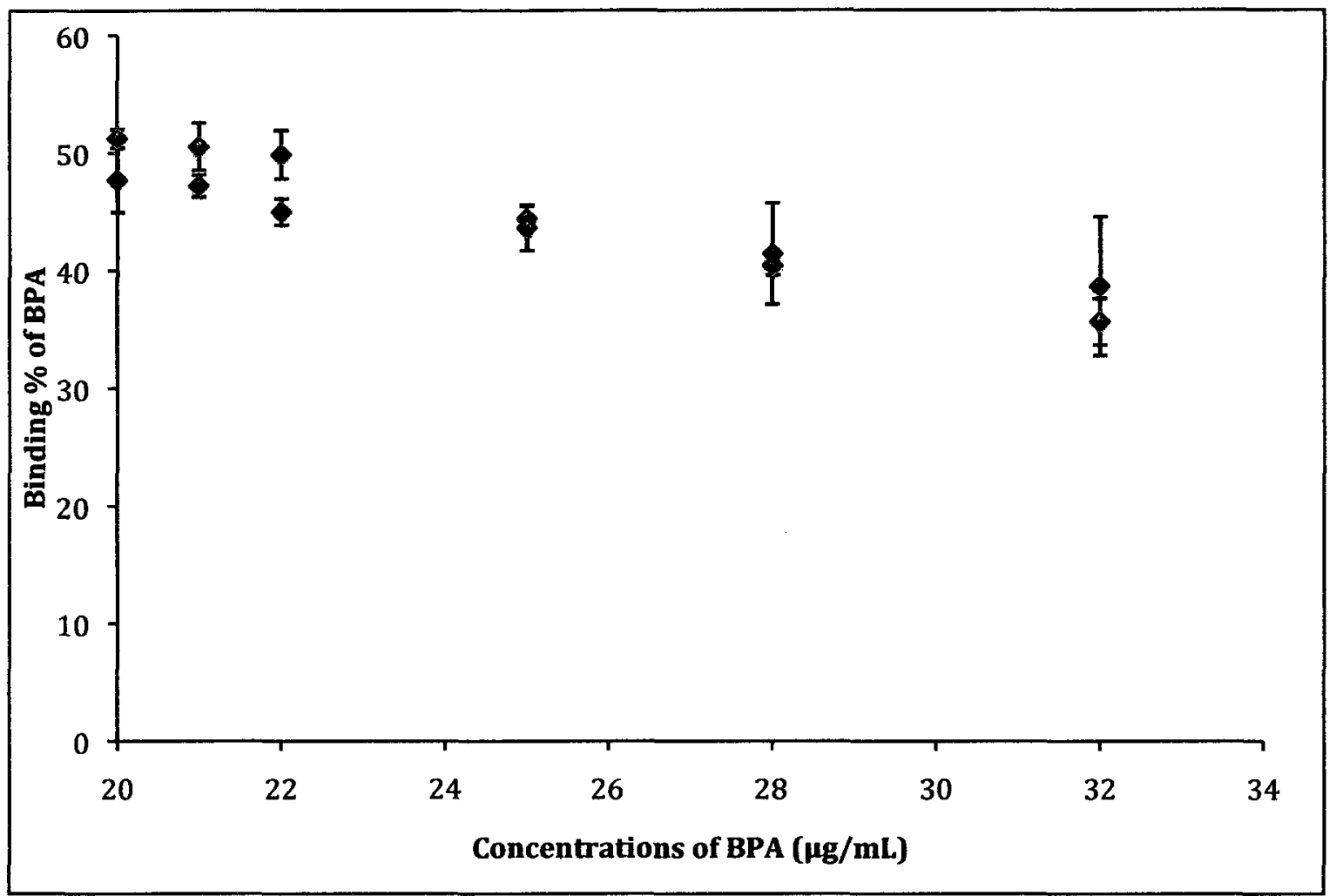

Figure 47 Binding efficiency of BPA on MIP-T7 $(\bullet)$ and NIP-T7 $(\bullet)$ in presence of different concentrations of BPA.

Table 13 Amount of bound BPA on MIP-T7 and NIP-T7 in presence of different concentrations of BPA.

\begin{tabular}{lll}
\hline Concentration of BPA $(\mu \mathrm{g} / \mathrm{mL})$ & $\begin{array}{l}\text { Bound PBA }(\mu \mathrm{mol} / \mathrm{g}) \\
\text { MIP-T7 }\end{array}$ & NIP-T7 \\
\hline 20 & $42 \pm 2$ & $45 \pm 1$ \\
21 & $43 \pm 1$ & $47 \pm 2$ \\
22 & $43 \pm 1$ & $48 \pm 2$ \\
25 & $49 \pm 1$ & $48 \pm 2$ \\
28 & $51 \pm 5$ & $50 \pm 1$ \\
32 & $54 \pm 8$ & $50 \pm 3$ \\
\hline
\end{tabular}

The binding efficiencies of BPA onto MIP-T4 and MIP-T7 particles were studied after addition of E2. Various concentrations of E2 were first added to the MIP particles. After 20 seconds, 
BPA was next added to attain a final concentration of $20 \mu \mathrm{g} / \mathrm{mL}$. The binding efficiency results of BPA on MIP-T4 particles were plotted (Figure 48). Addition of BPA to the particles in the absence of E2 (first point) showed a binding efficiency of $49 \pm 1 \%$. For the second data point, BPA was added after the MIP-T4 particles had 20 seconds to bind with $1 \mu \mathrm{g} / \mathrm{mL}$ E2 first. As a portion of specific binding sites $(3.6 \mu \mathrm{mol} / \mathrm{g})$ of the particles were occupied strongly by E2, the next added BPA molecules had to bind non-specifically with both high- and low-affinity binding sites. After addition of $2 \mu \mathrm{g} / \mathrm{mL} \mathrm{E} 2$ solution (for the third data point), most of the BPA molecules found only low-affinity sites on the MIP-T4 particles to bind with, resulting in relatively constant binding efficiencies of $31 \pm 2 \%$.

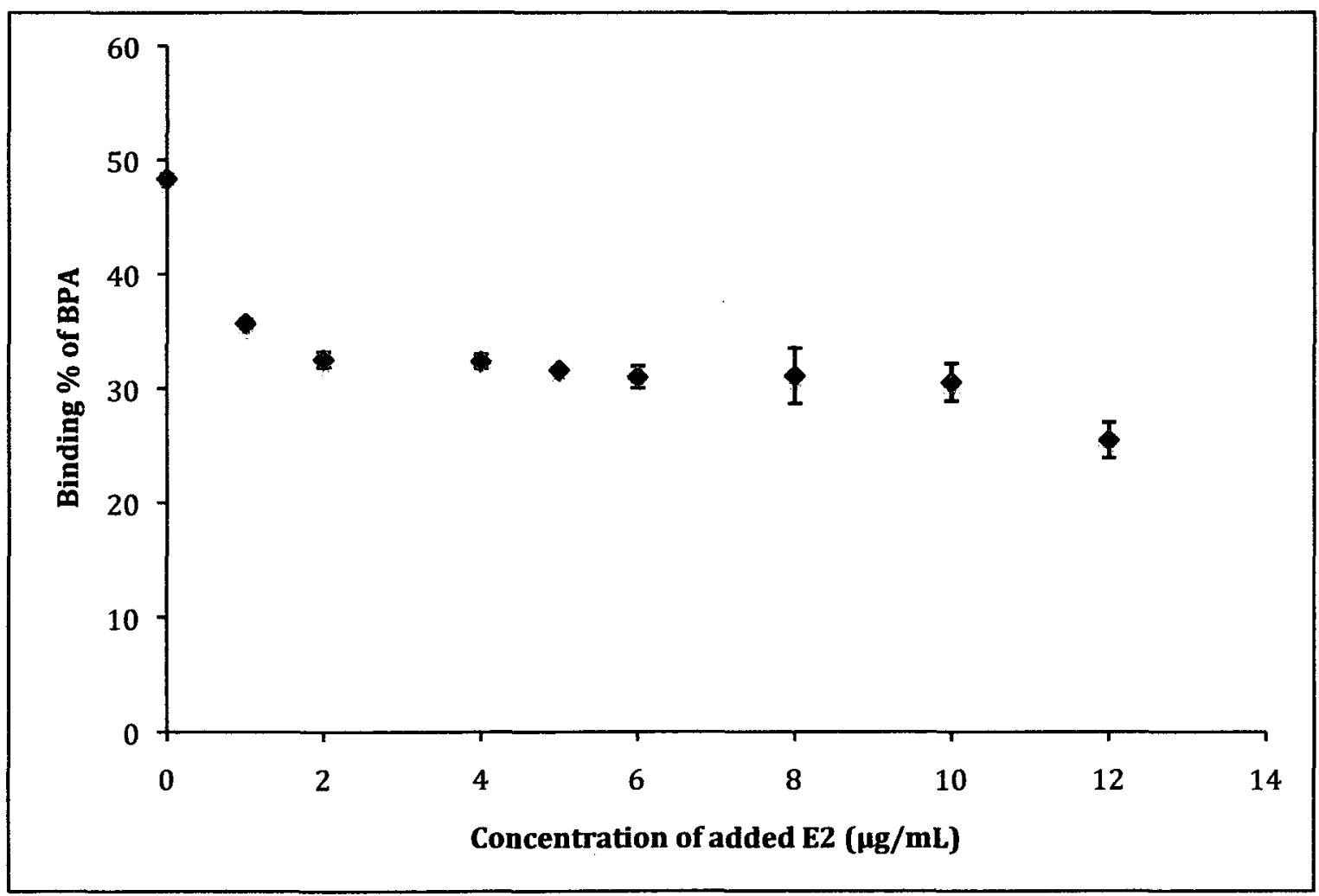

Figure 48 Binding efficiency of BPA on MIP-T4 particles, 20 seconds after addition of E2 at various concentrations. Concentration of BPA was $20 \mu \mathrm{g} / \mathrm{mL}$. 
The binding efficiency results of BPA on MIP-T7 particles are presented in Figure 49. As MIPT7 particles have a larger specific binding capacity $(43 \pm 8 \mu \mathrm{mol} / \mathrm{g})$ than that of MIP-T4 $(33 \pm 7$ $\mu \mathrm{mol} / \mathrm{g}$ ). In presence of more concentrations of E2 as a portion of specific binding sites of the particles were occupied strongly by E2, the next added BPA molecules had to bind nonspecifically with both high- and low-affinity binding sites. This were decreased the binding $\%$ of BPA while the concentration of E2 increased. It is noteworthy that (20 s) after addition of E2 at various concentrations from 2 to $10 \mu \mathrm{g} / \mathrm{mL}$, addition of $20 \mu \mathrm{g} / \mathrm{mL}$ BPA next still allowed $31 \pm 2 \%$ binding (of BPA). This means that all available E2 occupied only a small fraction of binding sites.

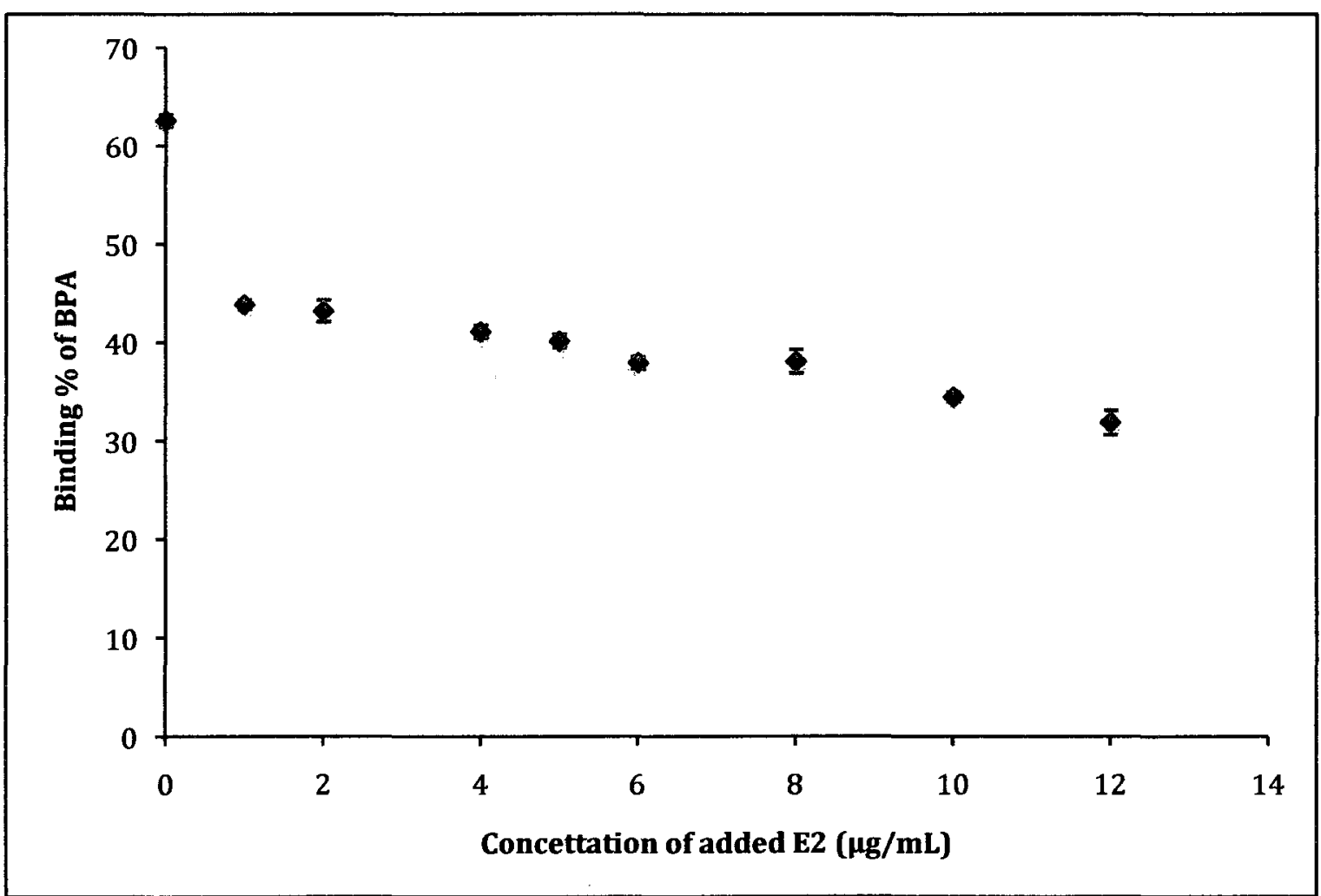

Figure 49 Binding efficiency of BPA on MIP-T7 particles, 20 seconds after addition of E2 at various concentrations. Concentration of BPA was $20 \mu \mathrm{g} / \mathrm{mL}$. 


\subsubsection{Binding competition of E2 and BPA on MIP-T4 and MIP-T7 particles}

$20 \mu \mathrm{g} / \mathrm{mL}$ of BPA was first introduced onto MIP-T4 and MIP-T7 particles. After $20 \mathrm{~s}$ of incubation, E2 was added at various concentrations to compete for the binding sites. As E2 bound more strongly to the specific sites in MIP-T4 particles the bound BPA molecules had to give up these sites for $1-2 \mu \mathrm{g} / \mathrm{mL}$ E2, as shown in Figure 50 . The binding \% of BPA continued to decrease only slightly while higher concentrations of E2 were added. Apparently, the bound BPA was able to keep E2 away from the non-specific sites. When $12 \mu \mathrm{g} / \mathrm{mL}$ E2 was added, the binding $\%$ of BPA decreased significantly to a low value of $15 \%$. One plausible explanation is that $44 \mu \mathrm{mol} / \mathrm{g}$ of E2 was able to force out most of the BPA which saturated the specific binding sites $(33 \pm 7 \mu \mathrm{mol} / \mathrm{g})$ of MIP-T4 particles.

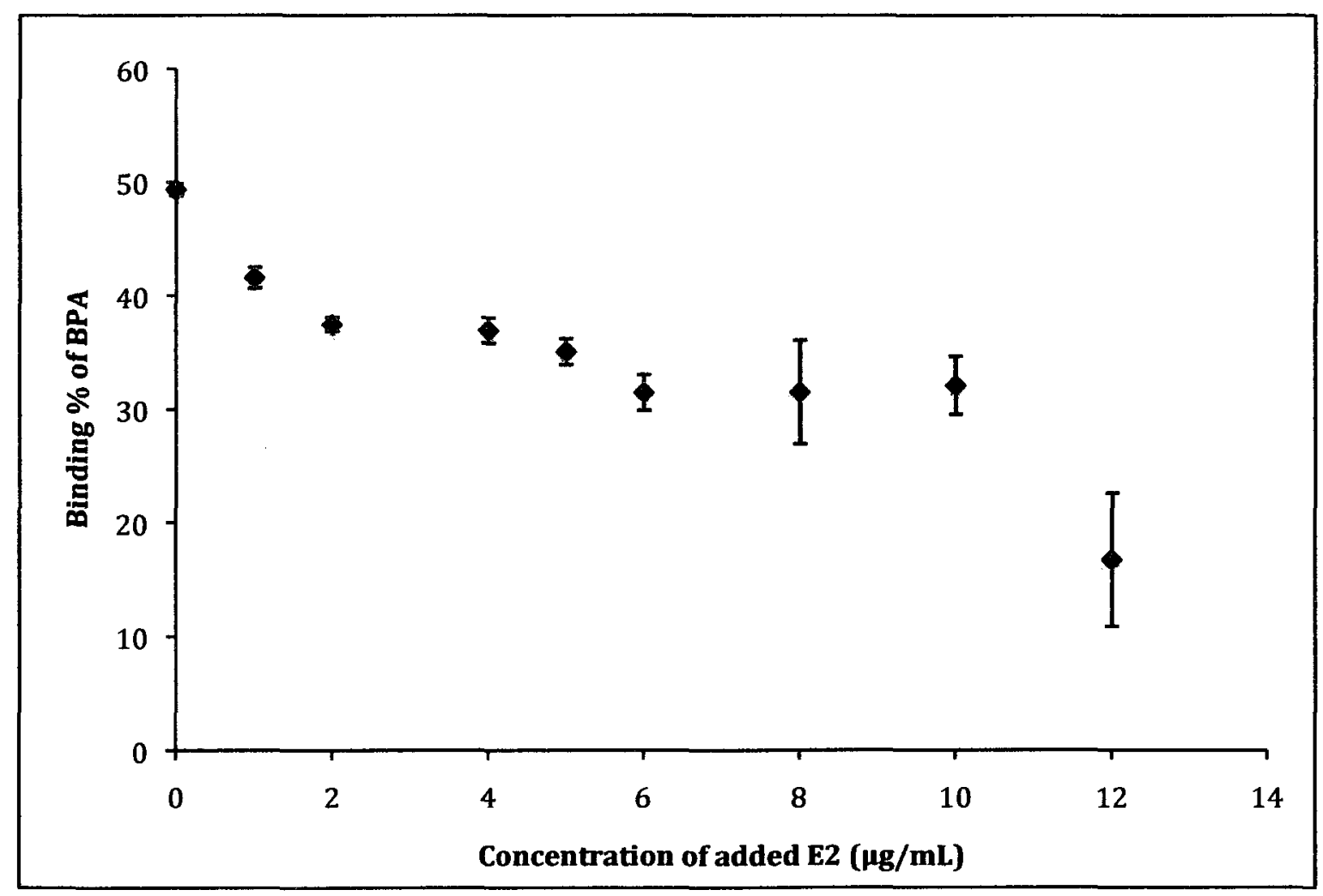

Figure 50 Binding efficiency of BPA $(20 \mu \mathrm{g} / \mathrm{mL})$ on MIP-T4 particles, determined after addition of E2 at various concentrations to compete for binding sites. 
For MIP-T7 particles, by adding $1 \mu \mathrm{g} / \mathrm{mL}$ of E2, the binding efficiency of BPA decreased (from $64 \%$ ) to $46 \%$, as shown in Figure 51. Apparently E2 bound more strongly to the specific sites, forcing the bound BPA molecules to give up these sites. When $2 \mu \mathrm{g} / \mathrm{mL}$ E2 was added, the binding \% of BPA did not decrease further. The large non-specific binding capacity for MIP-T7 particles resulted in a fairly constant binding efficiency of BPA when higher concentrations of E2 were added (from $2-8 \mu \mathrm{g} / \mathrm{mL}$ ). Beyond $8 \mu \mathrm{g} / \mathrm{mL}, \mathrm{E} 2$ addition caused the binding $\%$ of BPA to decrease. This means that E2 was able to displace bound BPA from the sites.

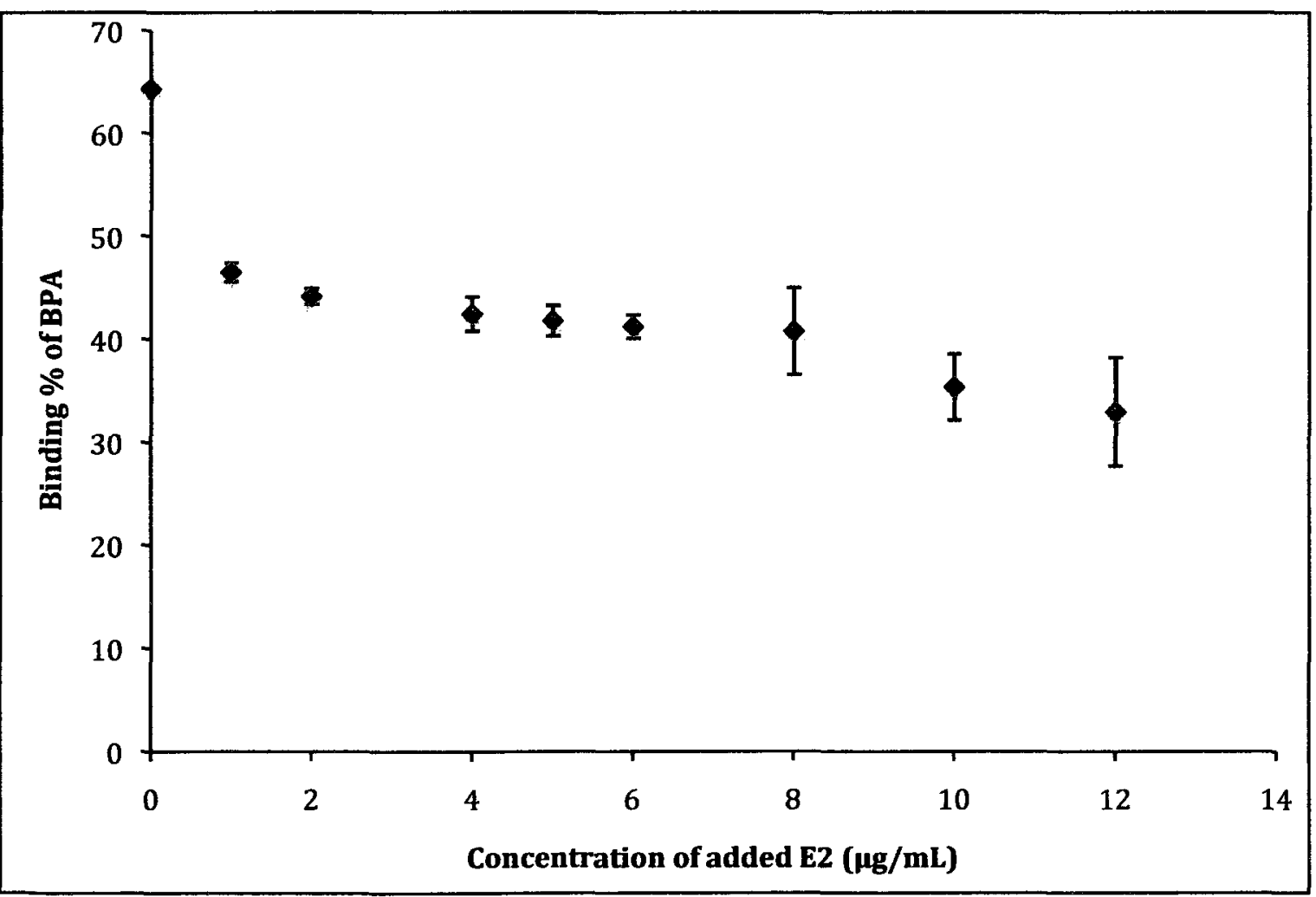

Figure 51 Binding efficiency of BPA $(20 \mu \mathrm{g} / \mathrm{mL})$ on MIP-T7 particles, determined after addition of E2 at various concentrations to compete for binding sites. 


\section{CHAPTER IV}

\section{CONCLUSIONS}




\section{Conclusions}

Extensive monitoring of intake and discharge waters is becoming a routine in water treatment plants. Cost-effective practices for removal of EDCs in water treatment will require both knowledgeable development and careful implementation. In the present study, E2 was chosen as a target molecule to develop an efficient and fast method for pre-concentration of EDCs in water (and potentially wastewater). For preparation of submicron MIP particles, a one-step precipitation polymerization method has been applied. Three different types of MIP submicron particles have been successfully prepared for E2. Using two different cross-linkers (EGDMA and TRIM) in varying ratios controlled the particle physical and binding properties. A smaller particle size of $282 \pm 4 \mathrm{~nm}$ was obtained where 1:8:7 mole ratios of template, functional monomer and 3-dimentional cross-linker (TRIM) were used. Batch binding study is a simple method for characterization of MIP particles, providing the first insight into their binding properties. Binding efficiencies of different MIP particles indicated that even after short incubation for 2 minutes, over $90 \%$ of E2 was bound. Between all MIP particles, MIP-T7 presented the best binding efficiency of $99.0 \pm 0.7 \%$.

Leakage of bound E2 from MIP particles into the aqueous solutions could not be detected, which made them a good sorbent for removal of E2 from water. As a fundamental investigation, Scatchard analysis gave information with respect to the mechanism of E2 adsorption on MIP particles. There are two types of adsorption sites, high-affinity (specific) sites and low-affinity (non-specific) sites. The overall experimental results on binding properties showed that MIP-T7 with specific binding capacity of $43 \pm 8 \mu \mathrm{mol} / \mathrm{g}$ and smallest diameter of 
$282 \pm 4 \mathrm{~nm}$ with imprinting effect close to 3 offers the best binding performance in comparison to other types of synthesized MIP.

A simple CE method was developed for the rapid characterization of both MIP and NIP particles, in contrast to other time-consuming techniques. These merits can be attributed to the use of BGE (50 mM borate buffer, $\mathrm{pH} 8.5$ ) with addition of a dynamic modifier polymer (EOTrol $\left.{ }^{\mathrm{TM}} \mathrm{LN}\right)$. The CE separation of three different types of MIPs and NIPs was based on their charge to size ratios $(q / r)$. Besides, DLS size analyzer, measured $r$ directly. Hence, CE and DLS can be used as a pair of techniques for fast determination of the charge density on submicron particles as it's non-specific binding sites compared to the NIP particles. These two properties ( $r$ and $q$ ) were interpreted as related to the non-specific binding sites on MIP and NIP particles.

Normally, selectivity of MIP particles for different analytes was individually studied. In this study, the selectivity of these particles for E2 was tested in the presence of BPA as a competitive molecule. Results on MIP-T4 and MIP-T7 indicated that both types of particles have a greater selectivity on E2 compared with accessible BPA. Although the selectivity of MIPs were found to be influenced adversely by the hydrophobic effect. However, the adsorption capacity of MIP particles was enhanced significantly. Moreover the study on selectivity of MIP articles indicated that E2 first bind on to the specific binding sites, then after saturation of them bind onto the non-specific binding sites of MIP particles. Meanwhile if other competition molecule occupied specific binding sites, as there is higher affinity for E2, they were replaced them. 


\section{Future work}

CE-UV emerges as a promising technique for the study of fast binding kinetics between polymeric particles and EDCs, such as E2 and BPA, down to a time scale in the order of a few seconds. The exact binding time is controlled by the relative mobility of particles with respect to analyte molecules, in two peak profiles. Faster binding kinetics of several seconds can be studied if the particles and molecules (are made to) carry opposite charges, and thus migrate in opposite directions. This possibility could open up a new area of research, wherein migration direction is dictated by a proper choice of $\mathrm{BGE}$ composition and $\mathrm{pH}$ to manipulate the ionic charge states of both the particles and analyte molecules. Such experiments are not totally novel in the literature, but are greatly facilitated by the simple design of CE. Any temperature adjustment is also easy to make when thermodynamic data become essential to a particular study of environmental ecology or wastewater treatment. 


\section{References}

1. Falconer, I. R.; Chapman, H. F.; Moore, M. R.; Ranmuthugala, G. Environ. Toxicol. 2006, 21, 181.

2. Besse, J. P.; Garric, J. Toxicol. Lett. 2008, 176, 104.

3. Rasier, G.; Toppari, J.; Parent, A. S.; Bourguignon, J. P. Mol.Cell. Endocrinol. 2006, 254-255, 187.

4. Cargouet, M.; Bimbot, M.; Levi, Y.; Perdiz, D. Environ. Toxicol. Pharmacol. 2006, 22, 104.

5. Cargouët, M.; Perdiz, D.; Mouatassim-Souali, A.; Tamisier-Karolak, S.; Levi, Y. Sci. Total Environ. 2004, 324, 55.

6. Darbre, P. D. Best Practice and Research Clinical Endocrinology and Metabolism, 2006, 20, 121.

7. Gomez, Y.; Valdez, R. A.; Larralde, C.; Romano, M. C.; J. Steroid Biochem. 2000, 74, 143.

8. Safe, S. H.; Pallaroni, L.; Yoon, K.; Gaido, K.; Ross, S.; Saville, B.; McDonnell, D. Reprod. Fertil. Dev. 2001, 13, 307.

9. Irmak, S.; Erbatur, O.; Akgerman, A. J. Hazard. Mater. B. 2005, 126, 54.

10. Lorphensri, O.; Intravijit, J.; Sabatini, D. A.; Kibbey, T.C.G.; Osathaphan, K.; Saiwan, C. Water Res. 2006, 40, 1481.

11. Auriol, M.; Filali-Meknassi, Y.; Tyagi, R. D.; Adams, C. D.; Surampalli, R.Y. Process Biochem. 2006, 41, 525.

12. Ahnerne, G. W.; English, J.; Marks, V. Ecotoxicol. Environ. Saf. 1985, 9, 79.

13. Desbrow, C.; Routledge, E. J.; Brighty, G. C.; Sumpte, J. P.; Waldock, M. Environ. Sci. Tech. $1998,32,1549$.

14. Shore, L. S.; Gurevitz, M.; Shemesh, M.; Bull. Environ. Contam. Toxicol. 1993, 361.

15. Alcock, R. E.; Sweetman, A.; Jones, K. C. Chemosphere 1999, 38, 2247.

16. Williams, R. J.; Jürgens, M. D.; Johnson, A. C. Wat. Res. 1999, 33, 1663.

17. Tschmelak, J.; Proll, G.; Gauglitz, G. Talanta, 2005, 65, 313.

18. Svenson, A.; Allard, A. S.; Ek, M. Water Res., 2003, 37, S19. 
19. Pawlowski, S.; Ternes, T. A.; Bonerz, M.; Rastall, A. C.; Erdinger, L.; Braunbeck, T. Toxicol. In Vitro. 2004, 18, 129.

20. D’Ascenzo, G.; Di Corcia, A.; Gentili, A.; Mancini, R.; Mastropasqua, R.; Nazzari, M.; Samperi, R.; Sci. Total Environ. 2003, 302, 199.

21. Johnson, A. C.; Sumpter, J. P. Environ. Sci. Technol. 2001, 35, 4697.

22. Ohko, Y.; Iuchi, K. I.; Niwa, C.; Tastuma, T.; Nakashima, T.; Iguchi, T.; Kubota, Y.; Fujishima, A. Environ. Sci. Technol. $2002,36,4175$.

23. Nghiem, L. D.; Schafer, A. I.; Environ. Eng. Sci. 2002, 19, 441.

24. Nghiem, L. D.; Schafer, A. I.; Waite, T. D. Desalination. 2002, 147, 269.

25. Labadie, P.; Hill, E. M. J. Chromatogr. A 2007, 1141, 174.

26. Andersson, L.; Paprica, A.; Arvidsson, T. Chromagraphia. 1997, 46, 57.

27. Walshe, M.; Howarth, J.; Kelly, M.; O’Kennedy, R.; Smyth, M. J. Pharmaceut. Biomed. Anal. 1997, 16, 319.

28. Matsui, J.; Okada, M.; Tsuruoka, M.; Takeuchi, T. Anal. Commun. 1997, 34, 85.

29. Le Noir, M.; Lepeuple, A. S.; Guieysse, B.; Mattiasson, B. Water Research, 2007, 41, 2825.

30. Jobling, S.; Casey, D.; Robert-Gray, T.; Oehlmann, J.; Schulte-Oehlmann, U.; Pawlowski, S.; Baunbeck, T.; Turner, A. P.; Tyler, C. R. Aquat. Toxicol. 2003, 65 (2), 205.

31. Lynn, S. G.; Birge, W. J.; Shepherd, B. S.; Comp. Biochem. Physiol. B, 2008, 149 (1), 126.

32. Ying, G. G.; Kookana, R. S.; Ru, Y. J. Envion. Int. 2002, 28 (6), 545.

33. Zamengo, L.; Barbiero, N.; Gregio, M.; Orrù, G. Chemosphere, 2009, 76, 313.

34. Le, T. T.; Saveyn, P.; Hoa, H. D.; Van der Meeren, P. Int. Dairy J. 2008, 18, 1090.

35. Wang, X. Z.; Liu. L.; Li, R. F.; Tweedie, R. J.; Primrose, K.; Corbett, J.; McNeil-Watson, F. K. Chem. Eng. Res. Des. 2009, 87, 874.

36. Deering, C. E.; Tadjiki, S.; Assemi, S.; Miller, J. D.; Yost, G. S.; Veranth, J. M. Particle and Fibre Toxicology, 2008, 5, 18.

37. Wang, M.; Bardajee, G. R.; Kumar, S.; Nitz, M.; Scholes, G. D.; Winnik, M. A. J. Chromatogr. A, 2009, 1216, 5011.

38. Singh, Y.; Lakshmy, R.; Gupta, R.; Kranthi, V. Lipids in Health and Disease, 2008, 7, 47.

39. Liu, F. K., J. Chromatogr. A, 2007, 1167, 231.

40. Lin, K. H.; Chu, T. C.; Liu, F. K. J. Chromatogr. A, 2007, 1161, 314. 
41. Whiting, C. E.; Arriaga, E. A. J. Chromatogr. A, 2007, 1157, 446.

42. Helle, A.; Hirsjärvi, S.; Peltonen, L.; Hirvonen, J.; Wiedmer, S. K. J. Chromatogr. A, 2008, 1178,248 .

43. d'Orlyé, F.; Varenne, A.; Gareil, P. J. Chromatogr. A, 2008, 1204, 226.

44. Candida, M.; Mellado, M.; Franco, C.; Coelho, A.; Alves, P.M.; Simplício, A. L. J. Chromatogr. A, 2008, 1192, 166.

45. Pereira, M.; Lai, E. P. C. J. Nanobiotechnology, 2008, 6, 10. doi:10.1186/1477-3155-6-10

46. Kuo, Y. C.; Lin, C. W. Colloids Surf. B, 2009, 72, 201.

47. Rodriguez-Santiago, V.; Fedkin, M. V.; Lvov, S. N. Rev. Sci. Instrum. 2008, doi:10.1063/1.2976779.

48. Chiu, J. H.; Peng, Y. N; Yang, Y. L.; Tsai, M. H.; Ho, Y. L.; Wu, C. Y.; Liu, M. Y. J. Chromatogr. $B, 2008,875,383$.

49. Rodriguez, M. A.; Armstrong, D. W. J. Chromatogr. B, 2004, 800, 7.

50. Garcia-Canas, V.; Cifuentes, A. Electrophoresis, 2007, 28, 4013.

51. Buszewski, B.; Klodzinska, E.; Dahm, H.; Roucki, H.; Szeliga, J.; Jackowski, M. Biomed. Chromatogr. 2007, 21, 116.

52. Pereira, M.; Lai, E.P.C.; Hollebone, B. Electrophoresis, 2007, 28, 2874.

53. Sellergren, B.; Shea, K. J. J. Chromatogr. 1993, 635, 31.

54. Chen, Y.; Kele, M.; Sajonz, P.; Sellergren, B.; Guiochon, G. Anal. Chem. 1999, 71, 928.

55. Andersson, L. I. J. Chromatogr. B Biomed. Appl. 2000, 739, 163.

56. Kriz, D.; Mosbach, K. Anal. Chim. Acta, 1995, 300, 71.

57. Kriz, D.; Ramstrom, O.; Mosbach, K. Anal. Chem. 1997, 69, 345A.

58. Kroger, S.; Truner, A. P. F.; Mosbach, K.; Haupt, K. Anal. Chem. 1999, 71, 3698.

59. Wulff, G.; Gross, T.; Schonfeld, R. Angew. Chem. Int. Ed. Engl. 1997, 36, 1961.

60. Yu, J. C. C.; Hrdina, A.; Mancini, C.; Lai, E.P.C. J. Nanoscience and Nanotechnol. 2007, 7, 3095 .

61. Sumi, V. S.; Kala, R.; Praveen, R. S.; Rao, T. P. Int. J. Pharm. 2008, 349, 30.

62. Wei, Y.; Qiu, L.; Owen, C.; Lai, E.P.C. Sens. Instrum. Food Qual. Saf. 2007, 1, 133.

63. Anderson, R. A.; Ariffin, M. M.; Cormack, P. A. G.; Miller, E. I. Forensic Sci. Int. 2008, 174,40 . 
64. Ge, Y.; Turner, A. P. F. Trends Biotechnol. 2008, 26, 218.

65. Pichon, V.; Chapuis-Hugon, F. Analytica Acta; 2008, 622, 48

66. Dickert F. L.; Tortschanoff, M,; Bulst, W. E.; Fischerauer, G. Anal. Chem. 1999, 71, 4559.

67. Sibrian- Vazquez, M.; Spivak, D.A. J. Polym. Sci. A., Polym. Chem. 2004, 42, 3668.

68. Glad, M.; Reinholdsson, P.; Mosbach, K. React. Polym. 1996, $25,47$.

69. Norrlöw, O.; Glad, M.; Mosbach, K. J. Chromatogr. 1984, 299, 29.

70. Wei, S.; Molinelli, A.; Mizaikoff, B. Biosensors and Bioelectronics, 2006, 21, 1943.

71. Dabek-Zlotorzynska, E.; Piechowski, M. Electrophoresis, 2007, $28,3526$.

72. Stevenson, D. TrAC, Trends Anal. Chem. 1999, 18, (3), 154.

73. Pyrzynska, K.; Pobozy, E.; Crit. Rev. Anal. Chem. 2002, 32, (3), 227.

74. Andersson, L. I. Bioseparation 2001, 10, (6), 353.

75. Mullett, W. M.; Pawliszyn, J. J. Sep. Sci. 2003, 26, (3/4), 251.

76. Zhang, Z.; Liu, Y.; Long, Y.; Nie, L.; Yao, S. Anal. Sci. 2004, 20, (2), 291.

77. Ye, L.; Weiss, R.; Mosbach; K. Macromolecules, 2000, 33, 8239.

78. de Boer, T.; Mol, R.; de Zeeuw, R. A.; de Jong, G. J.; Sherrington, D. C.; Cormack, P. A. G.; Ensing, K. Electrophoresis, 2002, 23, (9), 1296.

79. Li, P.; Rong, F.; Yuan, C. Polym. Int. 2003, 52, (12), 1799.

80. Piscopo, L.; Prandi, C. ; Coppa, M. ; Sparnacci, K. ; Laus, M. ; Lagana, A. ; Curini, R. ;D’Ascenzo, G. Imprinted. Macromol. Chem. Phys. 2002, 203, 1532.

81. Sanbe, H.; Haginaka, J. J. Pharm. Biomed. Anal. 2003, 30, 1835.

82. Zhonghbo, Z.; Hu, J. Water Research, 2008, 42, 4101.

83. Ye, L.; Yu, Y.; Mosbach, K. Analyst, 2001, 126, 760.

84. Rachkov, A.; McNiven, S.; El'skaya, A.; Yano, K.; Karube, I. Anal. Chim. Acta. 2000, 405, 23.

85. Ni, H.; Kawaguchi, H. J. Polym. Sci., A: Polym. Chem. 2004, 42, (11), 2833.

86. Andersson, L. I.; Paprica, A.; Arvidsson, T. Chromatographia, 1997, 46, 57.

87. Mullett, W.; Lai, E. P. C. Anal. Chem.,1998, 70, 3636.

88. Moller, K.; Crescenzi, C.; Nilsson, U. Anal. Bioanal. Chem., 2004, 387, 197.

89. Mastsui, J.; Miyoshi, Y.; Dolhoff,-Gior, O.; Takeuchi, T. Anal. Chem. 1995, 67, 4404.

90. Meng, Z.; Chen, W.; Mulchandani, A. Environ. Sci. Technol. 2005, 39, 8958. 
91. Kempe, M. Anal. Chem. 1996, 68, (11), 1948.

92. García-Calzón J.A.; Díaz-García, M. E. Sensors and Actuators B: Chemical, 2007, 123, 1180. 